\title{
Coupled Interface Modes for Nonlinear Interaction IN PERIOdiC LAYERED Media
}

by

\author{
Arghavan Arjmand
}

A thesis submitted in conformity with the requirements for the degree of Master of Applied Science and Engineering Graduate Department of The Edward S. Rogers Sr. Department of Electrical and Computer Engineering University of Toronto

Copyright (c) 2010 by Arghavan Arjmand 


\author{
Abstract \\ Coupled Interface Modes for Nonlinear Interaction \\ in Periodic Layered Media \\ Arghavan Arjmand \\ Master of Applied Science and Engineering \\ Graduate Department of The Edward S. Rogers Sr. Department of Electrical and \\ Computer Engineering \\ University of Toronto \\ 2010
}

This thesis proposes the platform for the observation of a new type of electromagnetic interface mode, Coupled Interface Mode, and studies the utilization of this mode in second order nonlinear interaction in AlGaAs. The dispersion relations for theoretical examination of the modes are developed and used to design a waveguide structure that accommodates a three wave mixing process utilizing coupled interface modes. The waveguides are fabricated according to optimized fabrication recipes and characterized for linear and nonlinear properties. Second harmonic generation is adopted for the demonstration of nonlinear interaction, due to its convenient experimental set-up. Three different laser sources are used to pump the waveguides and second harmonic light is generated and characterized. Coupled interface modes in conjunction with other types of modes also existing within the same structures, offer the possibility to explore three-wave mixing processes such as sum and difference frequency generation. 


\section{Acknowledgements}

I would like to express my deepest gratitude to Professor Amr Helmy without whom this study would not have been possible. I am grateful for his supervision, advice and guidance throughout the period of my research.

I also gratefully acknowledge Payam Abolghasem, Bhavin Bijlani and Junbo Han for their assistance and crucial contribution to my research.

I wish to state my sincere thanks to Dr. Henry Lee and Yimin Zhou from the Emerging Communications Technology Institute for their support in all of my fabrication work, as well as Battista Calvieri and Steven Doyle from the Microscopy Imaging Lab for their assistance with the Scanning Electron Microscopes.

Above all, I express my special thanks to my parents. Without their never ending love, support and dedication I would never be where I am now.

Arghavan Arjmand

September 2010 


\section{Contents}

1 Introduction $\quad 1$

1.1 Introduction to Thesis . . . . . . . . . . . . . 1

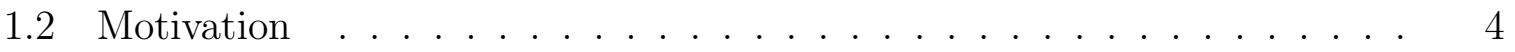

1.3 Modes at the Interface Between Two Media . . . . . . . . . . . . 6

1.4 Thesis Outline........................ 8

2 Interface Modes in Layered Media $\quad 10$

2.1 IMs at the interface of a semi-infinite periodic layered medium and a homogeneous medium . . . . . . . . . . . . . . . . . 10

2.2 IMs at the interface of two semi-infinite periodic layered media . . . . 15

2.3 IMs at the interface of a semi-infinite periodic layered medium with a cap layer and a homogeneous medium . . . . . . . . . . . . . 20

2.4 Proposal: IMs in a system of two semi-infinite periodic layered media with cap layers separated by a homogenous core . . . . . . . . . . . . 23

2.5 Coupled Interface Modes for Utilization in Nonlinear Interaction . . . . . 28

3 Phase Matching using interface modes 33

3.1 Phase-Matching the SHG process Using Interface Modes . . . . . . . . . 34

3.2 Mode Properties for Optimum Nonlinear Interaction . . . . . . . . . . 36

3.2.1 Nonlinear Conversion Efficiency . . . . . . . . . . . . 36

3.2.2 Group Velocity Mismatch . . . . . . . . . . . . . . 38 
3.2 .3 Group Velocity Dispersion . . . . . . . . . . . . . . . . . . 40

3.3 Performance of Coupled Interface Modes . . . . . . . . . . . . . . . 41

4 Wafer Design for Efficient SHG 45

4.1 Design Considerations . . . . . . . . . . . . . . . . . . . 45

4.2 Structure Design of Coupled Interface Modes for Type II phase matching 47

4.3 Structure Design of Coupled Interface Modes for Type I phase matching 48

4.4 Two dimensional Simulations of the CIM design . . . . . . . . . . . 51

5 Device Fabrication $\quad 56$

5.1 Wafer Growth . . . . . . . . . . . . . . . . . 56

5.2 Waveguide Fabrication . . . . . . . . . . . . . . 57

$5.2 .1 \quad$ Etch Mask Preparation _. . . . . . . . . . . . 57

$5.2 .1 .1 \quad$ Oxide layer deposition $\ldots \ldots \ldots \ldots$

5.2.1.2 Photoresist spin coating and Optical Lithography _. . 58

5.2.1.3 Plasma Etching of the Oxide Layer _ . . . . . . . 62

5.2 .2 Plasma Etching of the wafers . . . . . . . . . . . 64

5.2 .3 Wafer Thinning and Cleaving $\ldots \ldots \ldots \ldots \ldots$

$5.3 \quad$ Fabrication Obstacles . . . . . . . . . . . . . . . . . 68

6 Device Characterization $\quad 71$

6.1 Loss Measurements . . . . . . . . . . . . . . . . . . . . . . . 71

6.2 Observation of Second Order Nonlinear Interaction _ . . . . . . . . 72

7 Conlcusions $\quad 82$

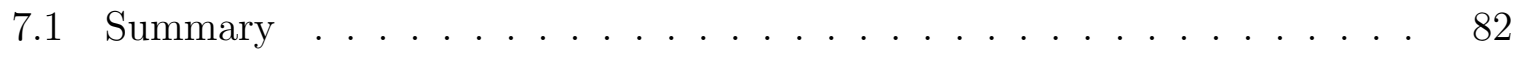

7.2 Future Work . . . . . . . . . . . . . . . . . . . 84 


\section{List of Figures}

1.1 A schematic of the cross section of a BRW . . . . . . . . . . . 5

2.1 Mode profiles of an interface mode at the boundary between a semi-infinite periodic layered medium and a homogeneous medium . . . . . . . . . 11

2.2 TE (top) and TM (bottom) band diagrams of guided states at the boundary between a semi-infinite periodic layered medium and a homogeneous

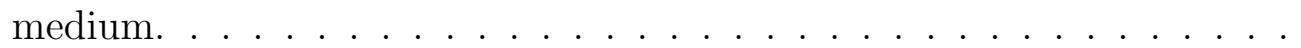

2.3 Mode profiles of an interface mode at the boundary between two semiinfinite periodic layered media $\ldots \ldots \ldots \ldots \ldots$

2.4 TE (top) and TM (bottom) band diagrams of guided states at the boundary between two semi-infinite periodic layered media. . . . . . . . . . 17

2.5 Profile of a mode that falls below the light line of the lowest refractive index material at the boundary between two semi-infinite periodic layered

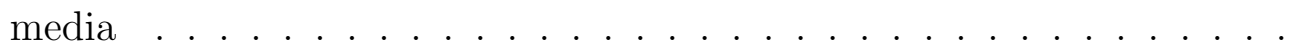

2.6 Mode profiles of an interface mode at the boundary between a semi-infinite periodic layered medium with a cap layer and a homogeneous medium . .

2.7 TE (top) and TM (bottom) band diagrams of guided states at the boundary between a semi-infinite periodic layered medium with a cap layer and

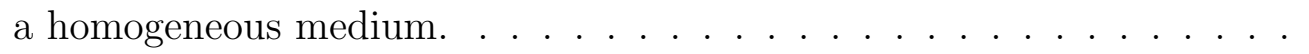


2.8 An interface mode profile at the boundary between two semi-infinite periodic layered media with cap layers separated by a homogenous medium

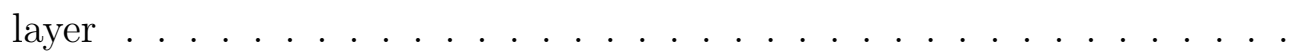

2.9 TE (top) and TM (bottom) band diagrams of guided states at the boundary between two semi-infinite periodic layered media with cap layers separated by a thick homogenous medium layer. . . . . . . . . . . . .

2.10 Mode profile of the TIR mode in the structure consisting of two semiinfinite periodic layered media with cap layers separated by a thin homo-

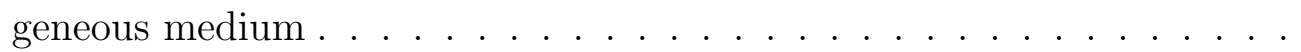

2.11 TE (left) and TM (middle) mode profiles of an interface mode at the boundary between two semi-infinite periodic layered media with cap layers separated by a thin homogenous medium layer; the index profile is also shown (right), the red dashed line designates the mode effective index. . .

2.12 TE (top) and TM (bottom) band diagrams of guided states at the boundary between two semi-infinite periodic layered media with cap layers separated by a thin homogenous medium layer. . . . . . . . . . . . . .

3.1 TIR and CIM mode profiles for type II phase matching . . . . . . . . . 36

3.2 Normalized coupling efficiency for type I and type II PM . . . . . . . . . 39

3.3 GVM as a function of cap and core layer aluminium concentrations . . . 40

3.4 TIR GVD as a function of cap and core layer aluminium concentrations . 41

3.5 CIM GVD as a function of cap and core layer aluminium concentrations

4.1 The intersection of the TE second harmonic wave refractive index and the average of TE and TM indices of the fundamental waves for type II phase matching. . . . . . . . . . . . . . . . .

4.2 Refractive index and mode profiles of TIR and CIM for type II phase matching . . . . . . . . . . . . . . . . . 
4.3 The intersection of the TE fundamental wave refractive index and TM second harmonic indices for type I phase matching. . . . . . . . . . . . . 50

4.4 Refractive index and mode profiles of TIR ad CIM for type I phase matching 50

4.5 Two dimensional mode profile of fundamental (right) and second harmonic (left) waves coexisting in the periodic layered medium. . . . . . . . . . 52

4.6 Type II phase matching wavelength shift for a 2D waveguide with a ridge width of $3 . \ldots \ldots \ldots$. . . . . . . . . . . . . . . . . 52

4.7 Type II phase matching wavelength shift for a 2D waveguide with a ridge width of $4 \mu m . \ldots \ldots \ldots \ldots$. . . . . . . . . . . . . . . . . 53

4.8 Type I phase matching wavelength shift for a 2D waveguide with a ridge width of $3 \mu m . \ldots \ldots \ldots \ldots \ldots$

4.9 Type II phase matching wavelength shift for a $2 \mathrm{D}$ waveguide with a ridge width of $5 \mu m . \ldots \ldots \ldots \ldots$. . . . . . . . . . . . . . 54

4.10 Type I phase matching wavelength shift for a $2 \mathrm{D}$ waveguide with a ridge width of $5 \mu \mathrm{m} . \ldots \ldots \ldots \ldots \ldots \ldots$

5.1 A schematic of the cross section of the structure . . . . . . . . . 57

5.2 SEMs of photoresist ridges as a function of development times . . . . . . 60

5.3 Schematic of a Mask Aligner. . . . . . . . . . . . . . . 61

5.4 SEM of an etch undercut on $\mathrm{SiO} 2$ formed due to an ICP overetch. . . . . 63

5.5 Photolithography Steps . . . . . . . . . . . . . . . . 64

5.6 SEM of etched GaAs using only photoresist. . . . . . . . . . . . . 65

5.7 A SEM of the wafer structure . . . . . . . . . . . . . 67

5.8 Schematic of Chemical Mechanical Polishing. . . . . . . . . . . . 68

5.9 Optical micrographs of wafer surface defects . . . . . . . . . . . 69

6.1 Schematic of the experimental setup for loss measurement . . . . . . . 72 
6.2 plot of the Fabry-Perot transmission spectrum of a $1.62 \mathrm{~mm}$ long waveguide; experimental measurements are shown in blue, theoretical fit is shown in red. . . . . . . . . . . . . . . . . . 73

6.3 Schematic of the SHG experimental setup . . . . . . . . . . . . . 74

6.4 SH power as a function of tuned pump wavelength . . . . . . . . . . . . 77

6.5 Spectra of pump and the corresponding SH for type II phase matching . 78

6.6 Quadratic relationship between the SH and pump powers . . . . . . . 79

6.7 Plot of the linear relationship between $\mathrm{SH}$ and pump power for type II phase matching . . . . . . . . . . . . . . . . . . 80 


\section{List of Tables}

3.1 Comparison of Mode Parameters for Different Multilayer Designs . . . . 43

3.2 Comparison of Mode Parameters for Different Multilayer Designs . . . 43

3.3 Comparison of Mode Parameters for Different Multilayer Designs . . . . 44

4.1 The Coupled Interface Mode design parameters for type II phase matching 49

4.2 The Coupled Interface Mode design parameters for type I phase matching 51

6.1 Comparison of Wave Parameters for Different Multilayer Designs. . . . . 77 


\section{Chapter 1}

\section{Introduction}

\subsection{Introduction to Thesis}

Laser sources have numerous practical and commercial applications such as multi-wavelength optical communications, optical metrology, spectroscopy, atmospheric remote sensing and image processing. Semiconductor lasers are of particular interest owing to their compactness, robustness, ease of maintenance and capability for monolithic integration; however, the output wavelength of practical semiconductor lasers fails to continuously cover the near and mid infrared region due to material band gap unavailability. Quantum cascade lasers are alternatives that cover a wide range of wavelengths from $3 \mu \mathrm{m}$ to tens of micrometers. For shorter wavelengths in the mid-infrared region however, optical parametric oscillators (OPO) become attractive sources of tunable coherent light. Many applications such as bio-photonics and sensing benefit from the output of such light sources. OPOs employ nonlinear optical interactions, most commonly second order nonlinearities, to produce near and mid infrared frequencies.

The most common medium used for nonlinear interaction in OPOs is Periodically Poled Lithium Niobate (PPLN). However, the need of careful optical tabletop alignment of the pump source and the nonlinear component in OPO devices limits their applicabil- 
ity [1]. Furthermore, the transparency window of PPLN, spanning only 350nm-4000 nm, confines their operations to those wavelengths. Via monolithic integration of an active laser source with the nonlinear component, where parametric oscillation takes place, this limitation can be eliminated. Such integrable nonlinear component will also benefit alloptical signal processing systems, metrology [2] and quantum communication schemes used for quantum key distribution (QKD) [3].

III-V semiconductors are attractive alternatives for integrated photonic devices due to their various enhanced properties over other materials. For example, in the case of Galium Arsenide (GaAs), there is one order of magnitude enhancement of second order nonlinearity ( $\chi^{2}$ value) over PPLN [1]. Also, in comparison to PPLN, GaAs spans a much larger transparency range from 1 to $17 \mu \mathrm{m}$. Semiconductors also benefit from high optical damage thresholds that render them suitable media for higher power nonlinear interactions. Another advantage of semiconductors arises from the mature fabrication technology which ultimately makes it possible to monolithically integrate semiconductor active and passive devices on one chip.

There is, however, a drawback associated with semicondcutors. Nonlinearities in semiconductors are maximized near the material band gap where the phase-matching (PM) condition is particularly difficult to meet due to material dispersion. This condition is necessary for ensuring efficient nonlinear processes and involves maintaining a proper phase relationship between the interacting waves in the direction of propagation, so that the amplitude contributions from different wave vectors add up constructively. As a result, developing a technique for phase-matching nonlinear interactions in semiconductors is a crucial step in the development of a low loss monolithically integrable platform for an efficient nonlinear interaction, and is of substantial importance to the field of photonics.

The optically isotropic nature of some highly nonlinear semiconductors such as GaAs inhibits their utilization in second order nonlinear processes. Several methods have been 
developed to match the momentum vectors of the interacting waves in semiconductors. Modal phase-matching is one such example [4]. In this method, a fundamental mode is phase- matched to a higher order mode within the same structure. While the ease of fabrication makes this technique more appealing, the great losses of the higher order mode, and small overlap integral between the two interacting modes, reduce the achievable efficienies.

Another method for achieving phase-matching for frequency mixing purposes is form birefringence [5]. In form birefringence, the isotropy of the non-birefringent crystal is broken by inserting thin oxidized aluminium arsenide layeres in the bulk material. Consequently, artificial birefringence is achieved in the resulting composite material, where the only nonlinear material is GaAs. While the insertion of the oxide layers renders the demonstration of frequency mixing possible in GaAs, it is going to hinder the monolithic integration of the nonlinear component with other active and passive components on the same chip. The existance of the oxide layers for instance would supress laser operation on the chip.

In another technique, quasi-phase-matching (QPM) [6], a periodically poled material is utilized to introduce an artificial wave vector to help satisfy the momentum conservation condition. In a periodically poled material, the orientation of the optical axis of the material is periodically inverted, hence inverting the sign of the second order nonlinear susceptibility, $\chi^{2}[7]$. This sign alternation introduces a fictitious wave vector which contributes to the momentum vector addition leading to a phase-matched process. So far, monolithic integration of the quasi-phase-matched nonlinear component with other components has been inefficient at best; one attempt to integrate a device phase-matched using QPM with other devices was accompanied by large losses and low nonlinearity [8].

The techniques mentioned above provide platforms that are sufficient for demonstration of tabletop optical parametric oscillators; however, they involve structures that do not allow for easy integration of the OPO with other active and passive devices. 
Recently studies have proposed a new technique for phase-matching $\chi^{2}$ processes in semiconductors via utilizing the dispersion properties of Bragg Reflection Waveguides (BRWs) [9, 10, 29]. The main advantage of this method over conventional methods is that it allows for monolithic integration with other active and passive devices while benefiting from low losses. In this technique, phase-matching is achieved between the two modes in a BRW which are guided by different mechanisms, namely, total internal reflection (TIR) and transverse Bragg reflection (TBR). Fig. 1.1 [12] shows a schematic of a BRW, where a TBR guided mode is phase-matched to a TIR guided mode for Second Harmonic Generation (SHG). The mode profiles are superimposed on the structure in the figure. The wave in dashed lines is the fundamental TIR guided wave, and the one shown with a solid line is the second harmonic TBR guided wave. $\omega_{\text {in }}, \omega_{\text {out }}$ and $2 \omega_{\text {out }}$ designate the TIR pump frequency, the TIR output frequency and the Bragg output second harmonic frequency, respectively.

While BRWs have provided a novel channel for phase-matching second order nonlinearities in semiconductors, the method remains open to further optimization via introducing additional parameters. In addition, other second order nonlinear processes such as sum and difference frequency generation can benefit from the extra degrees of freedom offered by a new type of mode to be phase-matched in a similar periodic layered medium.

The rest of this chapter will highlight the limitations of phase-matching using solely Bragg modes which will then serve as a motivation for the development of a new type of mode, namely, a coupled interface mode (CIM), to be utilized in conjunction with Bragg modes for second order nonlinear processes.

\subsection{Motivation}

Optimization of BRWs for utilization of semiconductor nonlinearities has been explored with the available structure design parameters such as layer thickness and refractive 


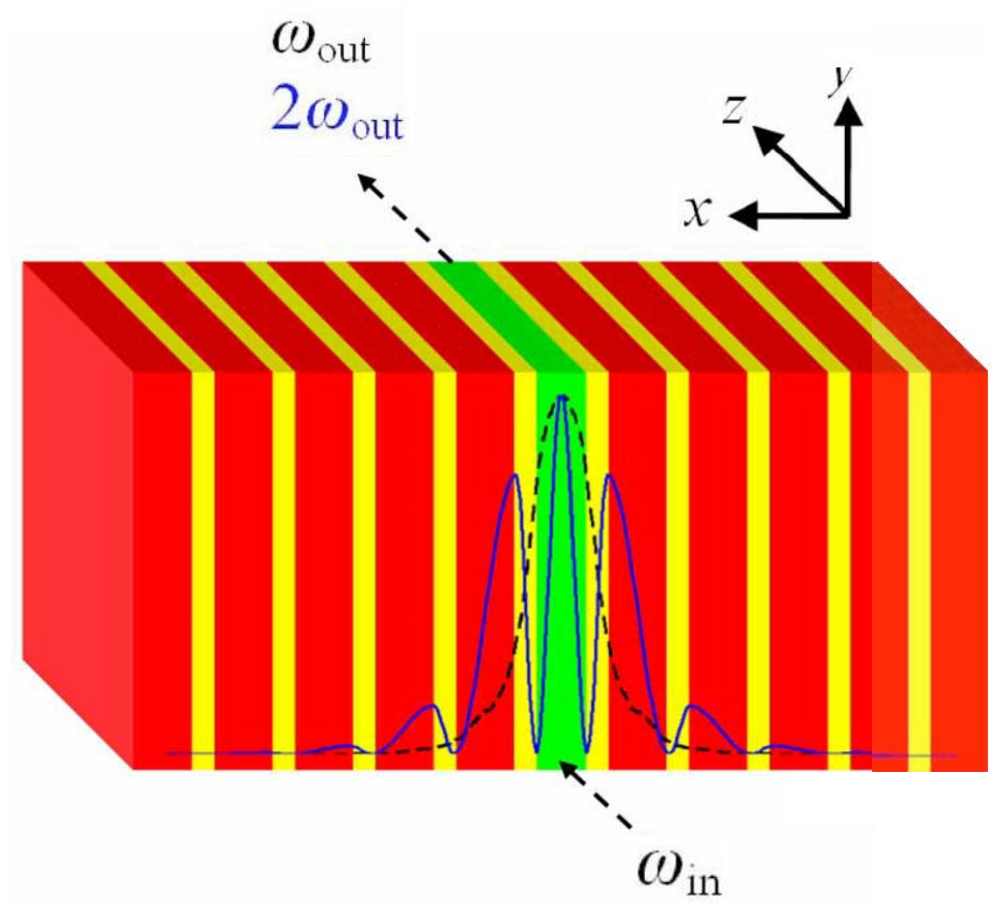

Figure 1.1 - A schematic of the cross section of a BRW; the fundamental (dashed line) and second harmonic (solid line) modes are superimposed on the structure.

indices [10, 29]; all of these studies have been examining operation at the quarter wave point, a condition that restricts the phases of the propagating waves in the structure layers and hence restricts the layer thicknesses. In conventional BRWs, this condition also implies a limitation on the thickness of the core layer of the structure. Furthermore, high nonlinear efficiencies are achieved at the cost of large temporal walk-off between the propagating waves which further lowers the overlap between the waves and hence the device efficiency. While abondoning the quarter wave condition might appear appealing due to the aformentioned restrictions, this condition offers advantages for which most structures are designed around it. The quarter-wave condition ensures closed-form design equations that are easy to develop and study. Also, closed-form expressions provide an insight into the optimization of the design and a direction for the optimization rather than a blind search. 
While operating at the quarter-wave point, introducing additional parameters into the design is one method to improve the device performance in BRWs. An example of such work is the utilization of matching layers in these structures $[9,13]$. The proposed method also operates at the quarter wave point; however, it eliminates the restrictions on the layer thicknesses as well as the trade-offs that exist between the device performance measures in a conventional BRW.

It would be very beneficial for second order nonlinear processes if a third type of mode, guided in a way other than TIR and TBR could exist in the very periodic layered media in which Bragg modes are studied. In the course of this thesis, a new type of mode named coupled interface mode will be proposed, developed, theoretically studied and experimentally observed for use in second order nonlinear interaction. The existance of this new mode allows for tuning of both linear and nonlinear wave properties while also permitting phase-matching between a TIR mode and the interface mode.

Coupled interface modes are attractive because they have much better mode profile overlap with TBR modes, than TIR modes do. Consequently, they provide a means for achieving three wave mixing processes other than SHG. Also, as will be discussed in the later chapters, CIMs much like the matching layer enhanced waveguides, remove the trade-offs between nonlinear device performance parameters in conventional BRWs.

The next section will review modes that exist at the interface of two media in order to develop some background on the coupled interface modes.

\subsection{Modes at the Interface Between Two Media}

Interface modes are electromagnetic waves that are supported at the boundary between two dissimilar media. Surface plasmon polaritons are interface modes that exist at the interface between a metal and a dielectric. These modes were among the first to be

investigated. Surface plasmon polaritons are utilized in various fields such as sensing [14] 
and microscopy [15]; however, the significant optical losses associated with these modes limit their utilization to very short propagation distances.

Another type of interface modes are the D'yakonov waves, predicted theoretically in 1988 [16] and demonstrated experimentally by Torner et al. [17]. These interface modes occur at the boundary between two transparent media and can take place in several configurations involving an isotropic material and a uniaxial or biaxial homogeneous medium, under positive birefringence conditions. Materials with such stringent requirements are not naturally available and require tailoring the structureral paramaters to meet the D'yakonov wave criteria [18]. Tamm modes are also interface modes that are supported by structurally chiral materials, relaxing the homogeneity condition in the direction normal to the interface [19]. Both D'yakonov and Tamm modes have potential applications in sensing as well as waveguiding; however, due to their restrictive material requirements, their monolithic integration with other devices is challenging.

Bloch interface modes are another type of interface modes, originally introduced in 1984 [20]. These waves are defined as modes with propagation that is locally confined to the interface between two semi infinite media [21], or at the surface of a semi-infinite periodic layered medium. The confinement of the surface mode in this case is due to the photonic band-gap effect of the periodic medium.

Bloch interface modes in their simplest form have been studied theoretically and have already been employed for applications such as bio-sensing [22]. Owing to their lossless nature and greater parameter space for dispersion tailoring, Bloch interface modes also offer the potential for being employed in applications such as dispersion engineering and vertical monolithic integration of active and passive photonic devices.

The same structures that accommodate Bragg reflection modes mentioned in 1.2, can also support modes that exist at the interface between the periodic claddings and the core. These interface modes could be utilized in a similar manner to achieve phasematched second order nonlinear processes providing extra degrees of freedom for further 
optimzation. In this thesis, the observation of a second order nonlinear process employing such coupled interface modes is reported.

\subsection{Thesis Outline}

In what follows, Bloch interface modes in different configurations will be reviewed. A new type of mode will be proposed and developed. This mode consists of two coupled interface modes, each formed at the boundary of a homogenous medium and a periodic layered medium. The field profile of CIMs look very similar to Bragg modes; this, as well as linear and nonlinear properties of this mode, make it a good candidate for use in nonlinear frequency conversion processes in conjunction with Bragg modes. In particular, second order nonlinear processes could be achieved in a structure that supports this interface mode as well as a guided mode where phase-matching between the two modes is realized. Second harmonic generation is the process used in the work of this thesis to prove the potential application of these modes in three wave mixing processes due to the less complex experimental set-up required for this process; nevertheless, since the phase-matching condition is very similar for SHG and other three wave mixing processes, this technique used for the demonstration of SHG can also be viable to other second order frequency conversion applications.

In this thesis, Coupled Interface Modes will be proposed and studied and the experimental observation of nonlinear interaction employing these modes will be reported. Chapter 2 will review a few cases of interface modes in periodic layered media providing band diagrams and dispersion relations, leading to the structures that support coupled interface modes. Chapter 3 will then discuss the possibility of nonlinear optical interaction utilizing coupled interface modes. In Chapter 4, one and two dimensional waveguides with a practical wafer growth will be designed. Chapter 5 will review the growth of the designed structure and the processes involved in ridge waveguide fabrication. Chapter 
6 will present the device characterization results and analyze them in accordance with theory. Lastly, chapter 7 will provide concluding remarks and discuss some future considerations regarding the device. 


\section{Chapter 2}

\section{Interface Modes in Layered Media}

In this section, interface modes that have already been reported in the literature are dicussed. These modes are used to form a novel type of guided mode akin to Bragg modes in BRWs. Four cases of Bloch interface modes are discussed: The boundary of a homogenous medium and a 1-D photonic crystal, the boundary of two 1-D photonic crystals, the boundary of a homogenous medium and a 1-D photonic crystal separated by a cap layer and the boundary of two 1-D photonic crystals separated by a cap layer and a homogenous core. The last case is further studied for use in second order nonlinear processes and in particular Second Harmonic Generation. In the review and development of the interface modes throughout this chapter, Yeh's formalism has been adopted [23].

\subsection{IMs at the interface of a semi-infinite periodic layered medium and a homogeneous medium}

The simplest case of electromagnetic Bloch surface modes occurs at the interface of a semi-infinite periodic layered medium and a homogenous medium. Fig. 2.1 depicts such a system. Taking the z-direction as the direction of propagation and assuming $\frac{\partial}{\partial y}=0$, the electric field satisfies the wave equation, 


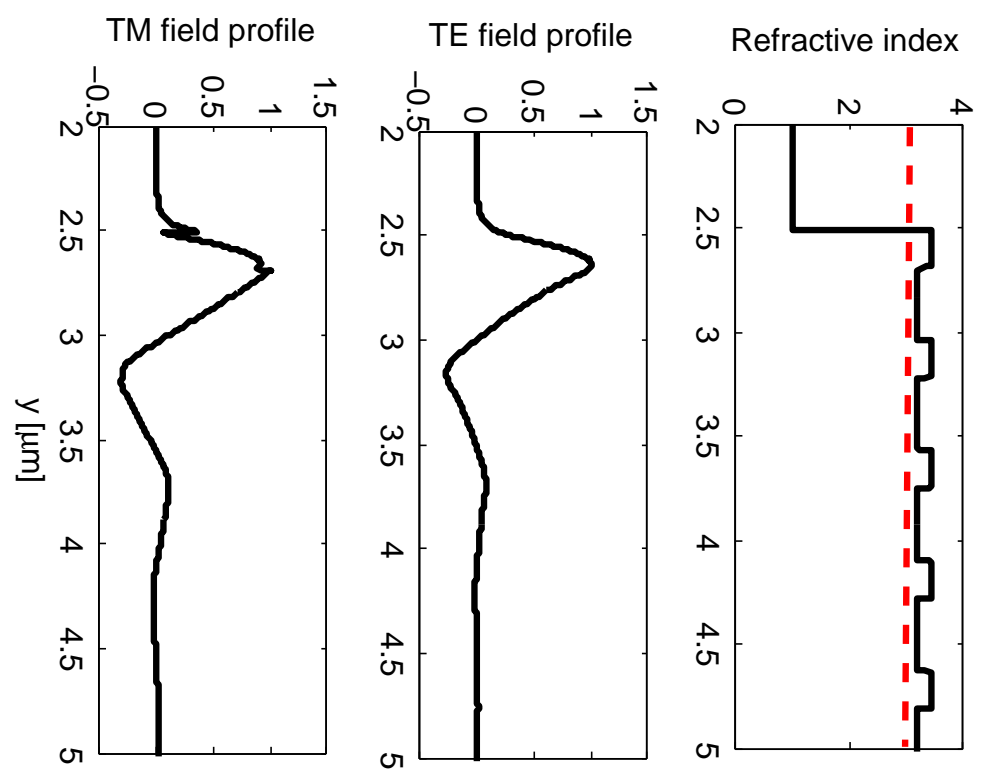

Figure 2.1 - TE (left) and TM (middle) mode profiles of an interface mode at the boundary between a semi-infinite periodic layered medium and a homogeneous medium; the index profile is also shown (right), the red dashed line designates the mode effective index.

$$
\frac{\partial^{2} E(x)}{\partial x^{2}}+k_{0}^{2} n^{2}(x) E(x)=\beta^{2} E(x)
$$

where $n(x)$ is the refractive index of the structure at location $x, \beta=k_{0} n_{e f f}, k_{0}$ is the free space wave vector and $n_{e f f}$ is the effective refractive index of the mode.

The electric field in a periodic medium can be written as,

$$
E(x)=\left\{\begin{array}{lr}
a_{n} e^{-i k_{1 x}(x-n \Lambda)}+b_{n} e^{i k_{1 x}(x-n \Lambda)}, & n \Lambda-a<x<n \Lambda \\
c_{n} e^{-i k_{2 x}(x-n \Lambda+a)}+d_{n} e^{i k_{2 x}(x-n \Lambda+a)}, & (n-1) \Lambda<x<n \Lambda-a
\end{array}\right.
$$

In the above equation, $a$ and $b$ are the thicknesses of the alternating layers 1 and 2, $\Lambda=a+b$ is the period. Also, $a_{n}, b_{n}, c_{n}, d_{n}$ are amplitude coefficients of the left and right propagating waves in the layers where $n$ is the order of the layer and

$$
k_{1 x}=k_{0} \sqrt{n_{1}^{2}-n_{e f f}^{2}}
$$




$$
k_{2 x}=k_{0} \sqrt{n_{2}^{2}-n_{e f f}^{2}}
$$

are the tangential wave vectors in the periodic layered medium.

According to the Floquet theorem, the wave equation in the semi-infinite periodic medium is of the form:

$$
E(x)=E_{\mathrm{K}}(x) e^{-i \mathrm{~K} \Lambda}
$$

where $E_{\mathrm{K}}(x)$ has period, $\Lambda=a+b$. This periodicity implies that the wave vector amplitudes at each unit cell are related by

$$
\left(\begin{array}{l}
a_{n} \\
b_{n}
\end{array}\right)=e^{-i \mathrm{~K} \Lambda}\left(\begin{array}{l}
a_{n-1} \\
b_{n-1}
\end{array}\right)
$$

Furthermore, the amplitudes of waves for adjacent unit cells are related by a translation matrix,

$$
\left(\begin{array}{ll}
A & B \\
C & D
\end{array}\right)\left(\begin{array}{l}
a_{n} \\
b_{n}
\end{array}\right)=\left(\begin{array}{l}
a_{n-1} \\
b_{n-1}
\end{array}\right)
$$

where the matrix entries are defined by

$$
\left\{\begin{aligned}
A & =e^{i k_{1 x} a}\left[\cos k_{2 x} b+\frac{1}{2} i\left(\frac{k_{2 x}}{k_{1 x}}+\frac{k_{1 x}}{k_{2 x}}\right) \sin k_{2 x} b\right] \\
B & =e^{-i k_{1 x} a}\left[\frac{1}{2} i\left(\frac{k_{2 x}}{k_{1 x}}-\frac{k_{1 x}}{k_{2 x}}\right) \sin _{2 x} b\right] \\
C & =B^{*} \\
D & =A^{*}
\end{aligned}\right.
$$

This summarizes the problem into an eigenvalue problem :

$$
\left(\begin{array}{ll}
A & B \\
C & D
\end{array}\right)\left(\begin{array}{l}
a_{n} \\
b_{n}
\end{array}\right)=e^{i \mathrm{~K} \Lambda}\left(\begin{array}{l}
a_{n} \\
b_{n}
\end{array}\right)
$$

where the eigen values and eigen vectors are given by Eq.2.9 and 2.10, respectively. 


$$
\begin{gathered}
e^{i \mathrm{~K} \Lambda}=\operatorname{Re}(A) \pm \sqrt{\operatorname{Re}(A)^{2}-1} \\
\left(\begin{array}{l}
a_{0} \\
b_{0}
\end{array}\right)=\left(\begin{array}{c}
B \\
e^{i \mathrm{~K} \Lambda}-A
\end{array}\right)
\end{gathered}
$$

The solution to the wave equation for the whole system is then written

$$
E(x)= \begin{cases}A_{0} e^{k_{h, \text { real }} x}, & x=0 \\ E_{\mathrm{K}}(x) e^{-i \mathrm{~K} x}, & x=0\end{cases}
$$

where $k_{h, \text { real }}=k_{0} \sqrt{n_{\text {eff }}^{2}-n_{h}^{2}}$ is the wave vector in the homogeneous medium and $A_{0}$ is the wave amplitude at the boundary between the homogeneous medium and the periodic layered medium. For example, the wave equation in the first layer of the periodic medium can be written

$$
E_{\mathrm{K}}(x)=\left(a_{0} e^{-i k_{1 x}(x-n \Lambda)}+b_{0} e^{i k_{1 x}(x-n \Lambda)}\right) e^{i \mathrm{~K}(x-n \Lambda)}
$$

The continuity of the wave equation and its derivative at the interface between the two materials implies

$$
\left\{\begin{array}{c}
A_{0}=a_{0}+b_{0} \\
k_{h, \text { real }} A_{0}=-i k_{1 x}\left(a_{0}-b_{0}\right)
\end{array}\right.
$$

Employing the continuity condition 2.13 and eliminating $A_{0}$ we get to the dispersion equation

$$
k_{h, \text { real }}=i k_{1 x} \frac{e^{i \mathrm{~K} \Lambda}-A-B}{e^{i \mathrm{~K} \Lambda}-A+B}
$$

Fig. 2.2 shows the band diagrams for transverse electric (TE) and transverse magnetic (TM) modes in this structure. The solid black lines are the light lines for the refractive indices of the system constituents; the top most line representing the lowest refractive index and the lower most line representing the highest refractive index.

Modes that are evanescent within the multilayer can exist in the forbidden bands of the structure as shown in red. No modes existing below the high-index light line, means that no guided modes propagate with an effective index greater than the largest index in the structure. Modes located in the light cone of the lowest-index material, the 

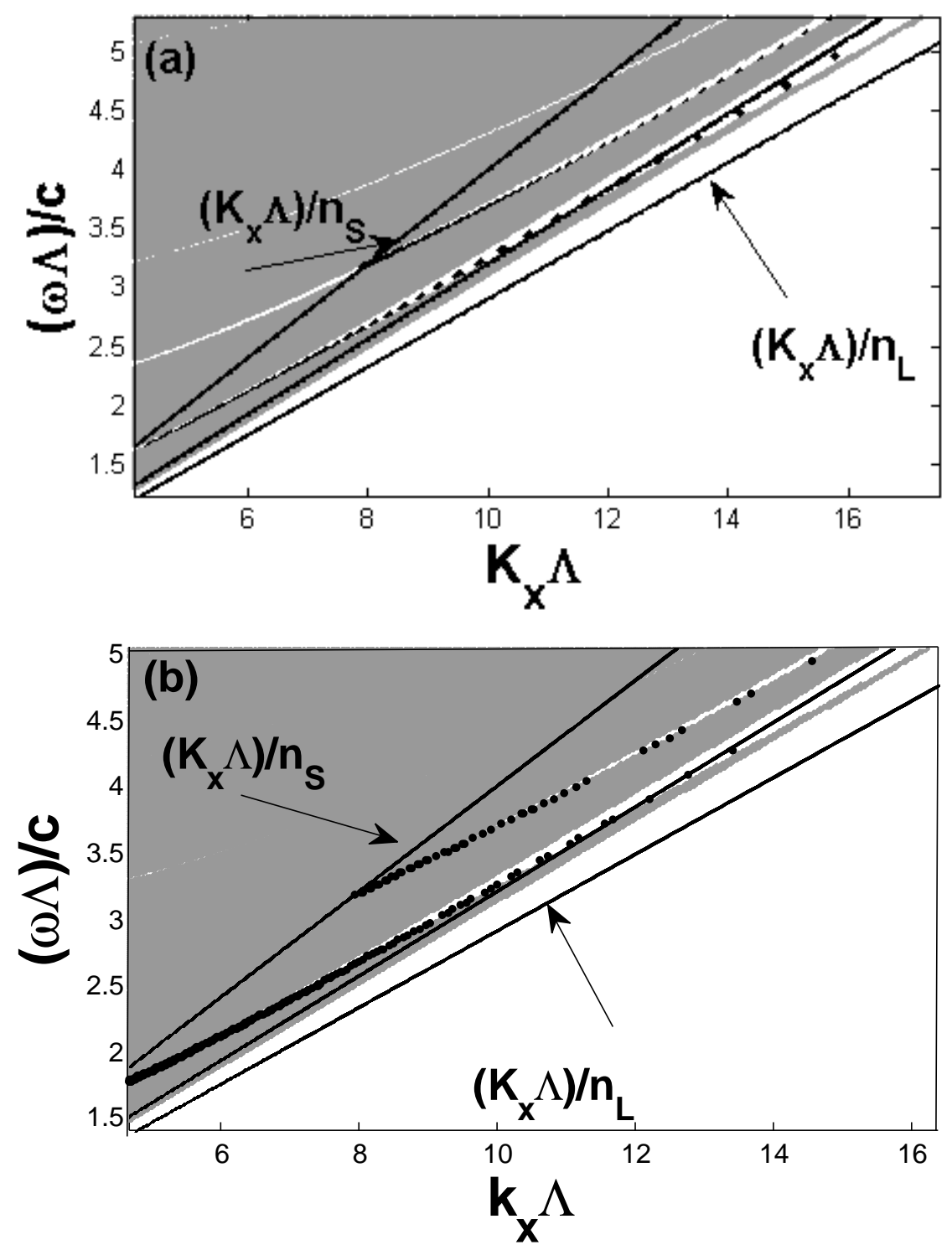

Figure 2.2 - TE (top) and TM (bottom) band diagrams of guided states at the boundary between a semi-infinite periodic layered medium and a homogeneous medium. 
homogenous medium in this case, are delocalized in that material. Modes below the light cone of the homogenous layer, are evanescent in that material and are hence localized at the interface between the homogenous material and the multilayer stack.

Surface modes at the boundary of a multi-layer stack and a homogenous medium have already been used for sensing applications [24, 25]. The use of surface interface modes for such applications offers several advantages over the conventional surface plasmon polariton modes. Materials capable of supporting surface plasmons suffer from significant optical losses which impedes the performance of devices that utilize these structures [26]. The losses deteriorate the device sensitivity and reduce the possibility of utilizing them for sensing. Bloch surface modes benefit from very low loss, rendering them a good fit for high sensitivity devices. Furthermore, the degrees of freedom offered by photonic bandgap materials, which arise from the ability of tuning the bandgap in relation to the operating wavelength, allows their use for sensing of a variety of materials and operation at a great range of wavelengths [26].

\subsection{IMs at the interface of two semi-infinite periodic layered media}

Interface modes can also take place at the boundary of two semi-infinite periodic layered media. Fig. 2.3 shows the geometry of such a system. Similar steps are followed to obtain the dispersion relation of the interface mode in this case.

In the right stack we have

$$
E(x)=\left\{\begin{array}{c}
a_{n} e^{-i k_{1 x}(x-n \Lambda)}+b_{n} e^{i k_{1 x}(x-n \Lambda)}, \quad(n+1) \Lambda-a<x<(n+1) \Lambda \\
c_{n} e^{-i k_{2 x}(x-n \Lambda+a)}+d_{n} e^{i k_{2 x}(x-n \Lambda+a)}, \quad n \Lambda<x<(n+1) \Lambda-a
\end{array}\right.
$$

and in the left stack, 


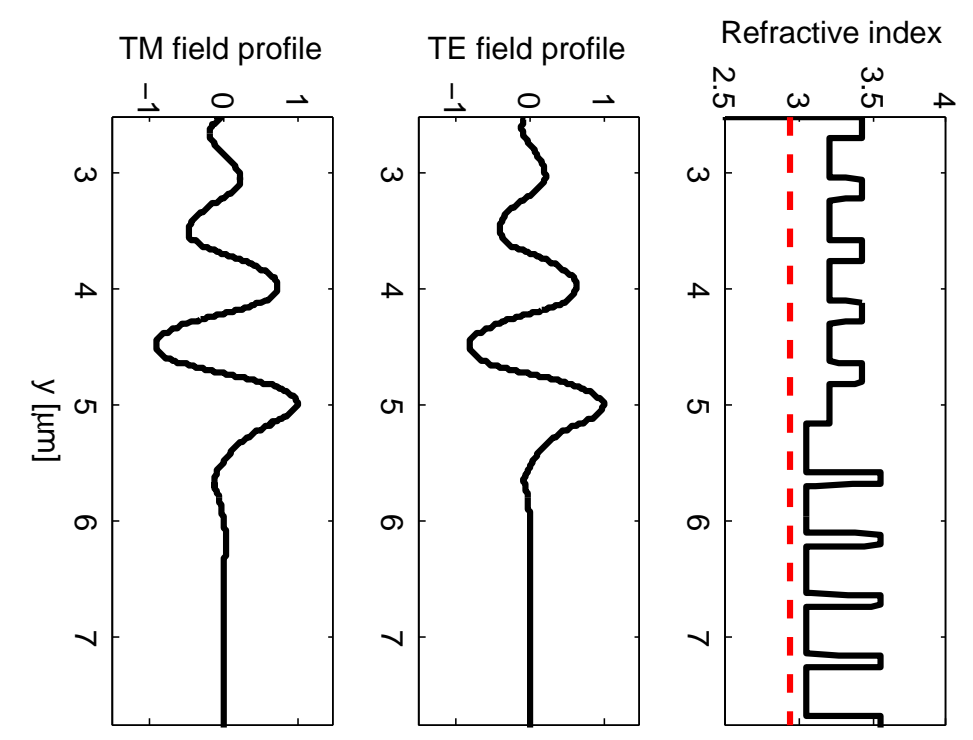

Figure 2.3 - TE (left) and TM (middle) mode profiles of an interface mode at the boundary between two semi-infinite periodic layered media; the index profile is also shown (right), the red dashed line designates the mode effective index.

$$
E(x)=\left\{\begin{array}{c}
c_{n}{ }^{\prime} e^{-i k_{3 x}\left(x+n^{\prime} \Lambda^{\prime}-c\right)}+d_{n}{ }^{\prime} e^{i k_{3 x}\left(x+n^{\prime} \Lambda^{\prime}-c\right)},-\left(n^{\prime}+1\right) \Lambda<x<-\left(n^{\prime}+1\right) \Lambda+c \\
a_{n}{ }^{\prime} e^{-i k_{4 x}\left(x-n^{\prime} \Lambda^{\prime}\right)}+b_{n}{ }^{\prime} e^{i k_{4 x}\left(x-n^{\prime} \Lambda^{\prime}\right)}, \quad-\left(n^{\prime}+1\right) \Lambda+c<x<-n^{\prime} \Lambda
\end{array}\right.
$$

In a similar manner to section 2.1 ,

$$
E_{\mathrm{K}}(x)=\left\{\begin{array}{c}
\left(a_{0} e^{-i k_{1 x}(x-n \Lambda)}+b_{0} e^{i k_{1 x}(x-n \Lambda)}\right) e^{i \mathrm{~K}(x-n \Lambda)}, x \in \text { layer } 1 \\
\left(a_{0}{ }^{\prime} e^{-i k_{4 x}\left(x-n^{\prime} \Lambda^{\prime}\right)}+b_{0}{ }^{\prime} e^{i k_{4 x}\left(x-n^{\prime} \Lambda^{\prime}\right)}\right) e^{i \mathrm{~K}^{\prime}\left(x-n^{\prime} \Lambda^{\prime}\right)}, x \in \text { layer } 4
\end{array}\right.
$$

where $a_{0}{ }^{\prime}, b_{0}{ }^{\prime}, n^{\prime}$ and $\Lambda^{\prime}$ are parameters of the left stack defined the same way as those for the right stack. Similarly by setting the boundary conditions equal at $x=0$, we get the dispersion relation:

$$
i k_{4 x} \frac{e^{i \mathrm{~K}^{\prime} \Lambda^{\prime}}-A^{\prime}-B^{\prime}}{e^{i \mathrm{~K}^{\prime} \Lambda^{\prime}}-A^{\prime}+B^{\prime}}=i k_{1 x} \frac{e^{i \mathrm{~K} \Lambda}-A-B}{e^{i \mathrm{~K} \Lambda}-A+B}
$$

Band diagrams of the guided modes in the particular structure of Fig. 2.3, are depicted in Fig. 2.4. The two light lines represent the highest and lowest index materials of the 

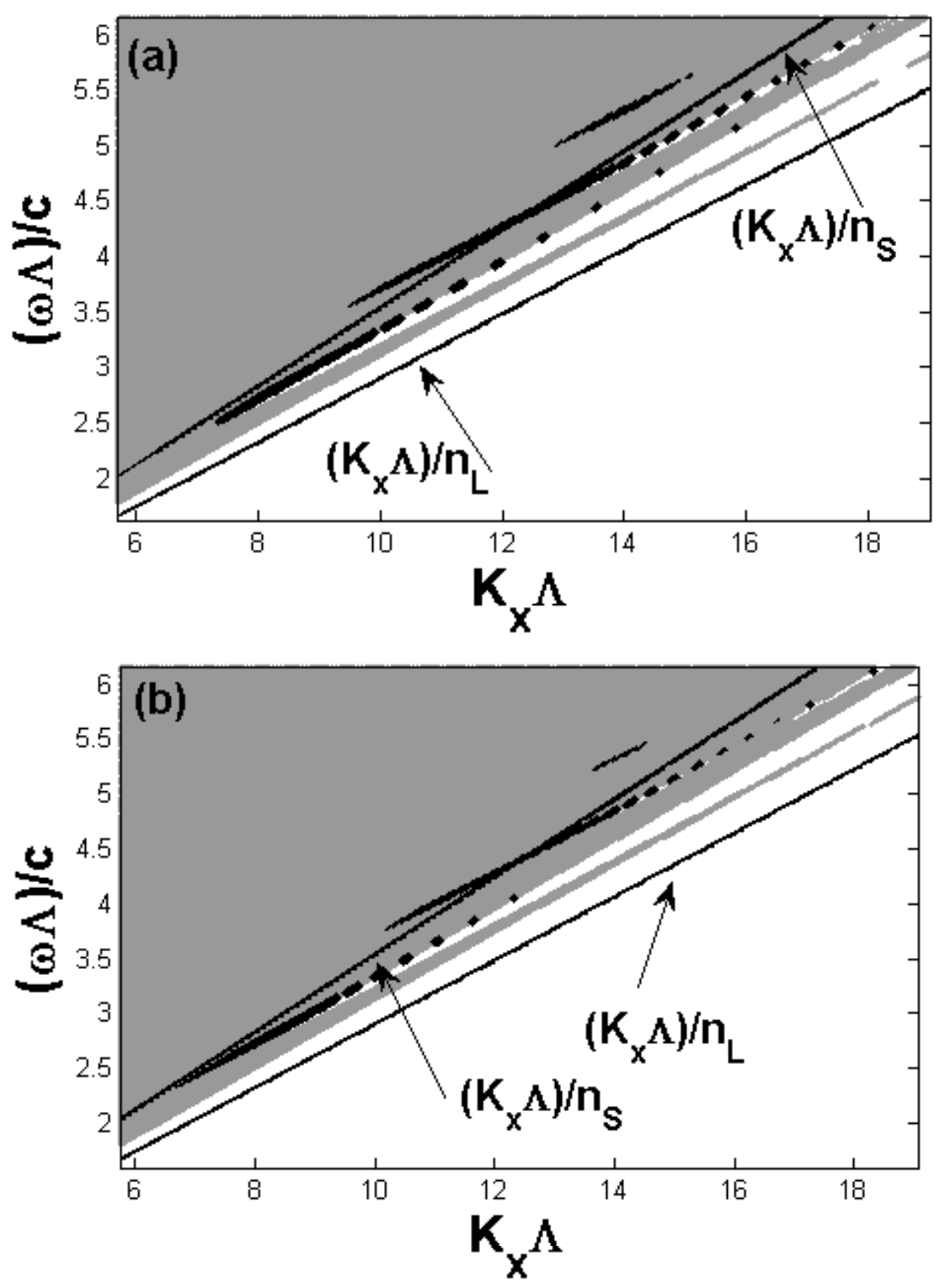

Figure 2.4 - TE (top) and TM (bottom) band diagrams of guided states at the boundary between two semi-infinite periodic layered media. 


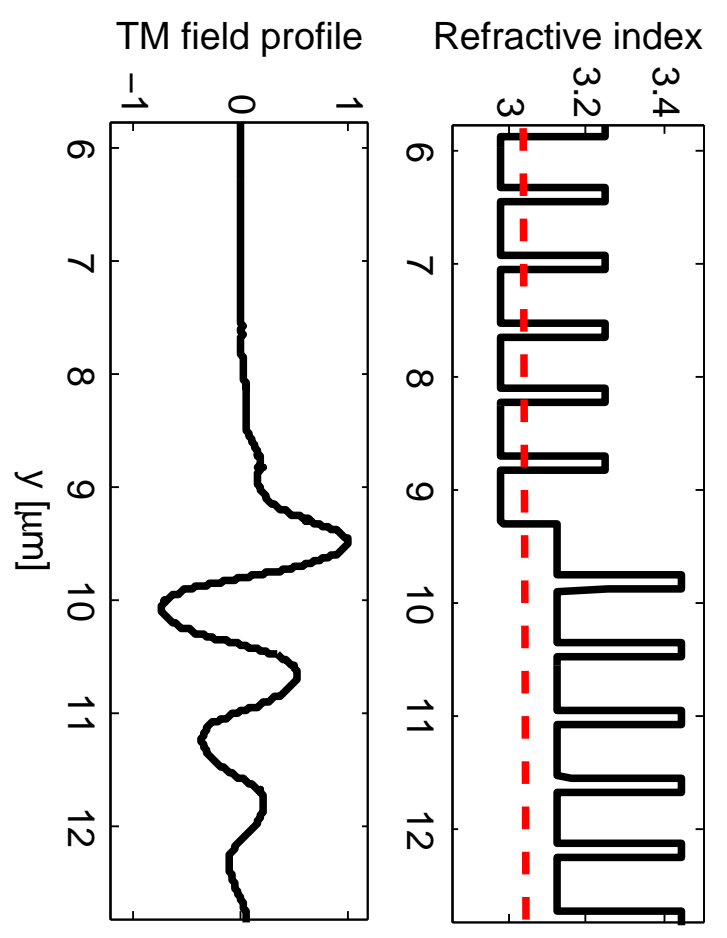

Figure 2.5 - Profile (left) of a mode that falls below the light line of the lowest refractive index material at the boundary between two semi-infinite periodic layered media; the index profile is also shown (right), the red dashed line designates the mode effective index.

system. Modes that fall within the light cone of the lowest refractive index material are interface modes confined to the interface of the two stacks. Modes that fall below this cone are also confined to the interface but are fully evanescent only in the stack with the higher index average and are only evanescent in the low index layers of the other stack. Fig. 2.5 shows the field profile of such a mode. The red dashed line shows the effective index of this particular mode.

Due to the particular geometry of this example, no Total Internal Reflection modes exist; however, if the first layers of the semi-infinite multilayers are replaced with highindex materials, TIR modes are bound to exist as well. These modes lie outside the bandgap of the photonic crystal and their effective index is greater than the two lowest indices of the structure.

Interface modes at the boundary of two periodic layered media have been studied 
for several applications. Villa [27] has studied modes peaking at the interface of two semi-infinite photonic crystal for design of narrow band-pass filters. A similar concept in photonic crystals with gain materials was used to design longitudinal mode selector of a laser.

Similar structures, where Bragg reflection waveguides take place [29] have already been utilized for edge-emitting diode lasers with the insertion of a quantum well layer at the core [30]. Interface modes at the boundary of two semi-infinite stacks can also serve the same purpose. Also, their comparable mode discrimination properties render the process of mode excitation desirably selective. The leakage loss value of the wave in the BRW in [30]was reported to be $0.1406 \mathrm{~cm}^{-1}$. Using the same method of [30], originally introduced by Ghatak et al. [31], loss values of a structure consisting of two semi-infinite multi-layers were obtained to be as low as $0.0043 \mathrm{~cm}^{-1}$. Since there is no core layer in such a system, for the same overall structure thickness, more periods could be added to the stack and hence a reduction of one order of magnitude could be achieved in the attainable losses, which can be very beneficial for high power laser operation.

In addition, the stacks can be terminated at either the low or high index layer. As such, when connecting two stacks to confine an IM, there are three possible permutations. With the right permutation in such system, interface and TIR modes can be utilized to achieve phase matching for second order nonlinear processes such as SHG and DFG, where the lower losses are again very advantageous. 


\subsection{IMs at the interface of a semi-infinite periodic layered medium with a cap layer and a homoge-} neous medium

Another variation of interface modes can occur at the boundary of semi-infinite periodic media with a high refractive index cap layer, and a homogeneous medium. Such a structure is capable of guiding Total Internal Reflection (TIR) waves as well as interface modes. The field equations inside the layers of this structure can be written as

$$
E(x)=\left\{\begin{array}{c}
A_{0} e^{k_{h, r e a l} x}, \quad x=0 \\
a_{c a p}^{+} e^{-i k_{c a p}\left(x-t_{c a p}\right)}+a_{c a p}^{-} e^{+i k_{c a p}\left(x-t_{c a p}\right)}, \quad 0=x=t_{c a p} \\
a_{n, 1}^{+} e^{-i k_{1}\left(x-t_{c a p}-n \Lambda+t_{2}\right)}+a_{n, 1}^{-} e^{+i k_{1}\left(x-t_{c a p}-n \Lambda+t_{2}\right)}, \quad t_{c a p}+(n-1) \Lambda=x=t_{c a p}+n \Lambda-t_{2} \\
a_{n, 2}^{+} e^{-i k_{2}\left(x-t_{c a p}-n \Lambda\right)}+a_{n, 2}^{-} e^{+i k_{2}\left(x-t_{c a p}-n \Lambda\right)}, \quad t_{c a p}+n \Lambda-t_{2}=x=t_{c a p}+n \Lambda
\end{array}\right.
$$

where $a_{n, j}^{+}$and $a_{n, j}^{-}$are the field amplitudes of the right and left propagating waves in the layer with refractive index $n_{j}$ and $a_{c a p}^{+}$and $a_{c a p}^{-}$are those of the travelling waves in the cap layer. Without loss of generality we'll take $A_{0}=1$ to normalize the field with respect to the field amplitude at the boundary of the cap and the homogenous medium.

By enforcing continuity at the boundary of the homogeneous material and the cap, we have

$$
\begin{aligned}
& a_{\text {cap }}^{+}=\frac{1}{2} e^{-i k_{\text {cap }} t_{\text {cap }}}+i \frac{1}{2} \frac{\mathrm{k}_{\mathrm{h}, \mathrm{real}}}{\mathrm{k}_{\mathrm{cap}}} e^{-i k_{\text {cap }} t_{\text {cap }}} \\
& a_{\text {cap }}^{-}=\frac{1}{2} e^{+i k_{\text {cap }} t_{\text {cap }}}-i \frac{1}{2} \frac{\mathrm{k}_{\mathrm{h}, \mathrm{real}}}{\mathrm{k}_{\text {cap }}} e^{+i k_{\text {cap }} t_{\text {cap }}}
\end{aligned}
$$

Now, assuming a QtW stack, it is predicted that at the boundary between the stack and the cap, there is an anti-node; therefore, the derivative of the wave function at this 
boundary is set to zero which leads to

$$
-i k_{c a p} a_{c a p}^{+}+i k_{c a p} a_{c a p}^{-}=0 \rightarrow a_{c a p}^{+}=a_{c a p}^{-}
$$

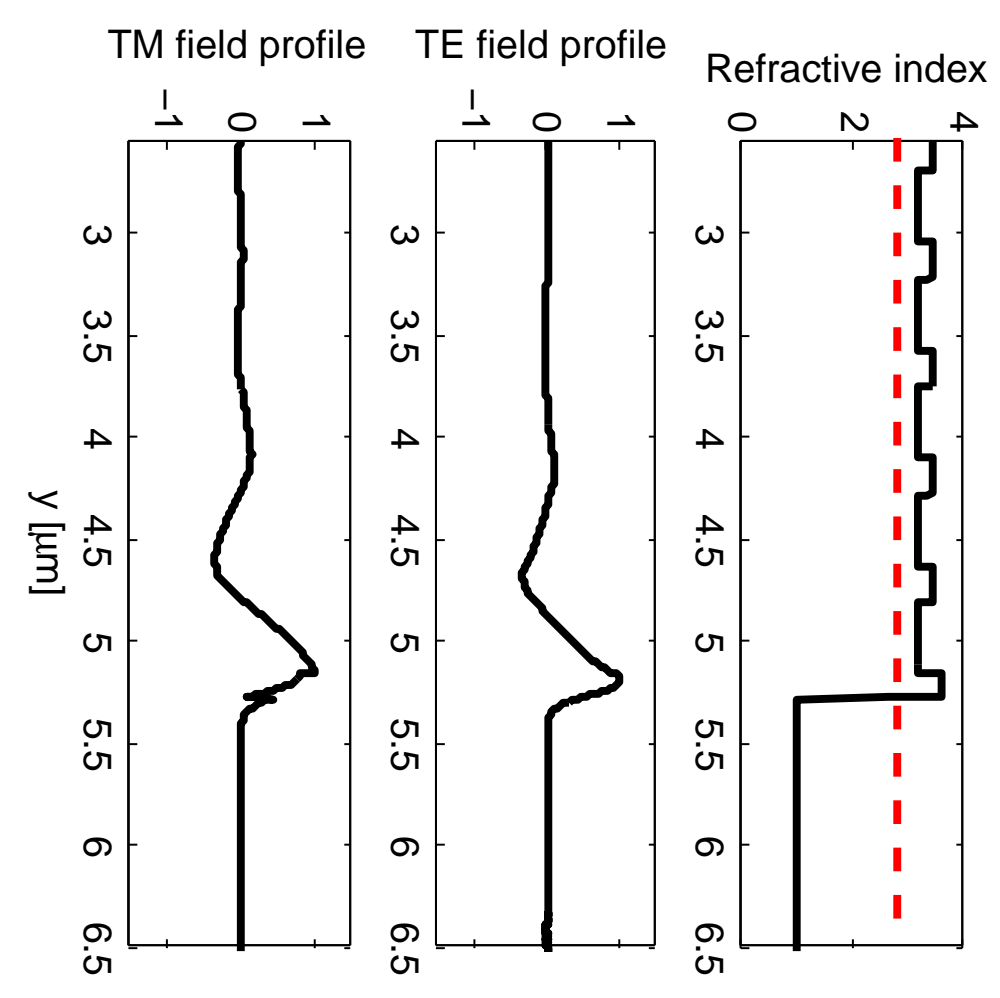

Figure 2.6 - TE (left) and TM (middle) mode profiles of an interface mode at the boundary between a semi-infinite periodic layered medium with a cap layer and a homogeneous medium; the index profile is also shown (right), the red dashed line designates the mode effective index.

Substituting Eq. 2.20a and 2.20b into Eq. 2.21, yields:

$$
\sin \left(k_{\text {cap }} t_{\text {cap }}\right)=\frac{\mathrm{k}_{\mathrm{h}, \text { real }}}{\mathrm{k}_{\text {cap }}} \cos \left(k_{\text {cap }} t_{c a p}\right) \rightarrow \tan \left(k_{\text {cap }} t_{c a p}\right)=\frac{\mathrm{k}_{\mathrm{h}, \text { real }}}{\mathrm{k}_{\text {cap }}}
$$

Eq. 2.22 is the dispersion relation for the structure in Fig. 2.6. Fig. 2.7 also shows the band diagrams for TE and TM modes in this structure. Both interface and TIR modes are present. TIR modes exist outside of the bandgap, while the interface modes are within 

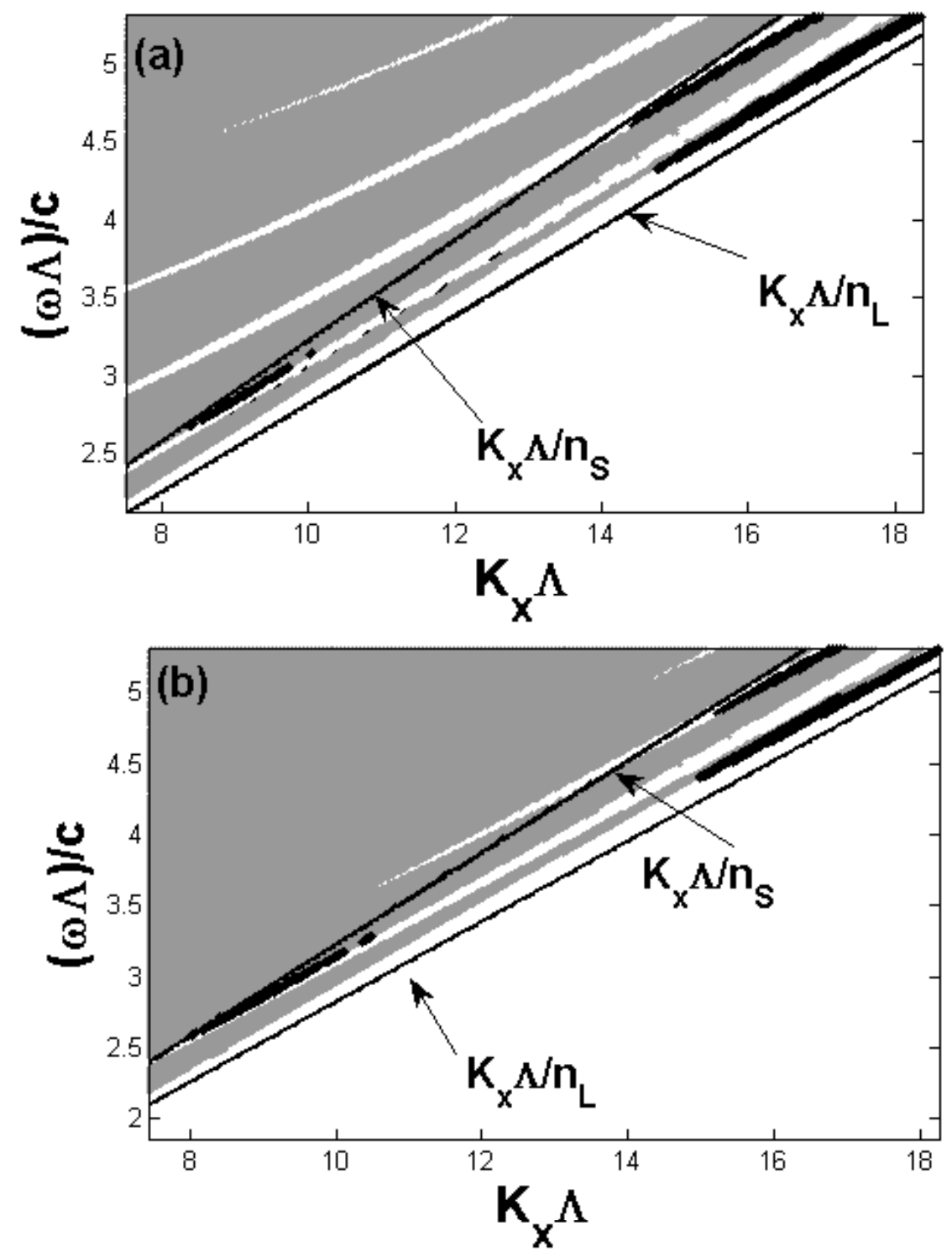

Figure 2.7 - TE (top) and TM (bottom) band diagrams of guided states at the boundary between a semi-infinite periodic layered medium with a cap layer and a homogeneous medium. 
the photonic bandgap of the periodic stack below the light cone of the homogenous medium, implying evanescence in the homogenous medium in agreement with the field distributions in Fig. 2.6.

These interface modes at the boundary of a homogenous medium and a multi-layer stack are also referred to as surface waves and can be used for the same applications as those of the waves in section 2.1; however, these structures benefit from the additional cap layer which provides extra degree of freedom in device design. It has been shown that the cap layer of a 1-D photonic crystal affects the position of surface modes in the band structure $[32,33]$ directing the dispersion properties of the structure. Studies have already taken advantage of this cap layer for more sensitive and more flexible sensors $[34,26,35]$.

\subsection{Proposal: IMs in a system of two semi-infinite periodic layered media with cap layers separated by a homogenous core}

In this section, the two periodic stacks of the last structure are brought together and the properties of the wave in that structure are examined to propose a new type of interface mode existing at the interfaces of the two stacks and the two cap layers. The homogenous medium from the structure in section 2.3 now plays the role of a "core" layer for this structure. Fig. 2.8 depicts this situation.

The wave equation for each side of the claddings is similar to Eq. 2.19. The field in the core is a hyperbolic cosine function consisting of the superposition of two exponentials: 


$$
\left\{\begin{array}{cr}
A_{0} \cosh \left(\mathrm{k}_{\mathrm{c}, \mathrm{real}} x\right), & 0 \leq x \leq \frac{t_{c}}{2} \\
a_{c a p}^{+} e^{-i k_{c a p}\left(x-t_{c a p}-\frac{t_{c}}{2}\right)}+a_{c a p}^{-} e^{+i k_{c a p}\left(x-t_{c a p}-\frac{t_{c}}{2}\right)}, \quad \frac{t_{c}}{2} \leq x \leq t_{c a p}+\frac{t_{c}}{2} & \\
a_{n, 1}^{+} e^{-i k_{1}\left(x-t_{c a p}-\frac{t_{c}}{2}-n \Lambda+t_{2}\right)}+a_{n, 1}^{-} e^{+i k_{1}\left(x-t_{c a p}-\frac{t_{c}}{2}-n \Lambda+t_{2}\right)}, & t_{c a p}+\frac{t_{c}}{2}+(n-1) \Lambda \leq x \leq t_{c a p}+\frac{t_{c}}{2}+n \Lambda-t_{2} \\
a_{n, 2}^{+} e^{-i k_{2}\left(x-t_{c a p}-\frac{t_{c}}{2}-n \Lambda\right)}+a_{n, 2}^{-} e^{+i k_{2}\left(x-t_{c a p}-\frac{t_{c}}{2}-n \Lambda\right)}, & \\
t_{c a p}+\frac{t_{c}}{2}+n \Lambda-t_{2} \leq x \leq t_{c a p}+\frac{t_{c}}{2}+n \Lambda
\end{array}\right.
$$

where $k_{j}=k_{0} \sqrt{n_{j}^{2}-n_{e f f}^{2}}, j=1,2$, cap, $c$ and $\mathrm{k}_{\mathrm{c}}=i \mathrm{k}_{\mathrm{c}, \text { real }}$.

Now by employing the continuity condition at the boundary of the core material and the cap, we have

$$
\begin{aligned}
& a_{\text {cap }}^{+}=\frac{1}{2} \cosh \left(k_{c, \text { real }} \frac{t_{c}}{2}\right) e^{-i k_{c a p} t_{c a p}}+i \frac{1}{2} \frac{\mathrm{k}_{\mathrm{c}, \mathrm{real}}}{\mathrm{k}_{\mathrm{cap}}} \sinh \left(k_{c, \text { real }} \frac{t_{c}}{2}\right) e^{-i k_{\text {cap }} t_{c a p}} \\
& a_{\text {cap }}^{-}=\frac{1}{2} \cosh \left(k_{c, \text { real }} \frac{t_{c}}{2}\right) e^{+i k_{\text {cap }} t_{c a p}}-i \frac{1}{2} \frac{\mathrm{k}_{\mathrm{c}, \mathrm{real}}}{\mathrm{k}_{\mathrm{cap}}} \sinh \left(k_{c, \text { real }} \frac{t_{c}}{2}\right) e^{+i k_{\text {cap }} t_{c a p}}
\end{aligned}
$$

and for the TM polarization,

$$
\begin{aligned}
& a_{\text {cap }}^{+}=\frac{1}{2} \cosh \left(k_{c, \text { real }} \frac{t_{c}}{2}\right) e^{-i k_{c a p} t_{c a p}}-i \frac{1}{2} \frac{\mathrm{k}_{\mathrm{c}, \mathrm{real}}}{\mathrm{k}_{\mathrm{cap}}} \frac{\mathrm{n}_{c a p}^{2}}{\mathrm{n}_{c}^{2}} \sinh \left(k_{c, \text { real }} \frac{t_{c}}{2}\right) e^{-i k_{\text {cap }} t_{c a p}} \\
& a_{\text {cap }}^{-}=\frac{1}{2} \cosh \left(k_{c, \text { real }} \frac{t_{c}}{2}\right) e^{+i k_{\text {cap }} t_{c a p}}+i \frac{1}{2} \frac{\mathrm{k}_{\mathrm{c}, \mathrm{real}}}{\mathrm{k}_{\mathrm{cap}}} \frac{\mathrm{n}_{c a p}^{2}}{\mathrm{n}_{c}^{2}} \sinh \left(k_{c, \text { real }} \frac{t_{c}}{2}\right) e^{+i k_{\text {cap }} t_{c a p}}
\end{aligned}
$$

Again, assuming a QtW stack, at the boundary between the stack and the cap, there is an anti-node; therefore, the derivative of the wave function at this boundary is set to zero and so we have

$$
-i k_{c a p} a_{c a p}^{+}+i k_{c a p} a_{c a p}^{-}=0 \rightarrow a_{c a p}^{+}=a_{c a p}^{-}
$$


Substituting the set of Eq. 2.24 into Eq. 2.26, yields:

$$
\begin{aligned}
& \tanh \left(\frac{\mathrm{k}_{\mathrm{c}, \text { real }} t_{c}}{2}\right) \tan \left(k_{\text {cap }} t_{c a p}\right)=-\frac{k_{\text {cap }}}{\mathrm{k}_{\mathrm{c}, \text { real }}} \\
& \left\{\tanh \left(\frac{\mathrm{k}_{\mathrm{c}, \text { real }} t_{c}}{2}\right) \cot \left(k_{\text {cap }} t_{c a p}\right)=\frac{k_{\text {cap }}}{\mathrm{k}_{\mathrm{c}, \text { real }} *}\right.
\end{aligned}
$$

It is worth noting that as $t_{c} \rightarrow \infty$ in the above expression, we get Eq. 2.22 of the structure in section 2.3. These dispersion relations can also be obtained by substituting the real equivalent of the core wave vector in equations 15 and 16 in [9] since $\mathrm{k}_{\mathrm{c}}=i \mathrm{k}_{\mathrm{c} \text {,real }}$.

Similarly for the TM polarization we have

$$
\begin{aligned}
& \operatorname{coth}\left(\frac{k_{c, \text { real }} t_{c}}{2}\right) \tan \left(k_{\text {cap }} t_{\text {cap }}\right)=+\frac{k_{c, \text { real }}}{k_{\text {cap }}} \frac{n_{\text {cap }}^{2}}{n_{c}^{2}} \\
& \tanh \left(\frac{k_{c, \text { real }} t_{c}}{2}\right) \tan \left(k_{\text {cap }} t_{\text {cap }}\right)=-\frac{k_{\text {cap }}}{k_{c, \text { real }}} \frac{n_{c}^{2}}{n_{\text {cap }}^{2}}
\end{aligned}
$$

From the above equations the cap layer thickness for a QtW structure can be extracted

$$
\mathrm{t}_{\text {cap }}=\frac{1}{\mathrm{k}_{\text {cap }}} \tan ^{-1}\left[-\frac{\mathrm{k}_{\text {cap }}}{\mathrm{k}_{\mathrm{c}, \text { real }}} \operatorname{coth}\left(\mathrm{k}_{\mathrm{c}, \text { real }} \frac{\mathrm{t}_{\mathrm{c}}}{2}\right)\right]+\mathrm{p} \pi
$$

and for TM polarization,

$$
\begin{aligned}
& \mathrm{t}_{\text {cap }}=\frac{1}{\mathrm{k}_{\text {cap }}} \tan ^{-1}\left[\frac{\mathrm{n}_{\text {cap }}^{2}}{\mathrm{n}_{\mathrm{c}}^{2}} \frac{\mathrm{k}_{\text {cap }}}{\mathrm{k}_{\mathrm{c}, \text { real }}} \tanh \left(\mathrm{k}_{\mathrm{c}, \text { real }} \frac{\mathrm{t}_{\mathrm{c}}}{2}\right)\right]+\mathrm{p} \pi \\
& \mathrm{t}_{\text {cap }}=\frac{1}{\mathrm{k}_{\text {cap }}} \tan ^{-1}\left[-\frac{\mathrm{n}_{\mathrm{c}}^{2}}{\mathrm{n}_{\text {cap }}^{2}} \frac{\mathrm{k}_{\text {cap }}}{\mathrm{k}_{\mathrm{c}, \text { real }}} \operatorname{coth}\left(\mathrm{k}_{\mathrm{c}, \text { real }} \frac{\mathrm{t}_{\mathrm{c}}}{2}\right)\right]+\mathrm{p} \pi
\end{aligned}
$$

These equations can be used to design QtW stack layers that accommodate both TIR and interface modes. With appropriate design parameters, these modes can be phase matched for a second order nonlinear optical process such as Frequency Down Conversio or Second Harmonic Generation. 


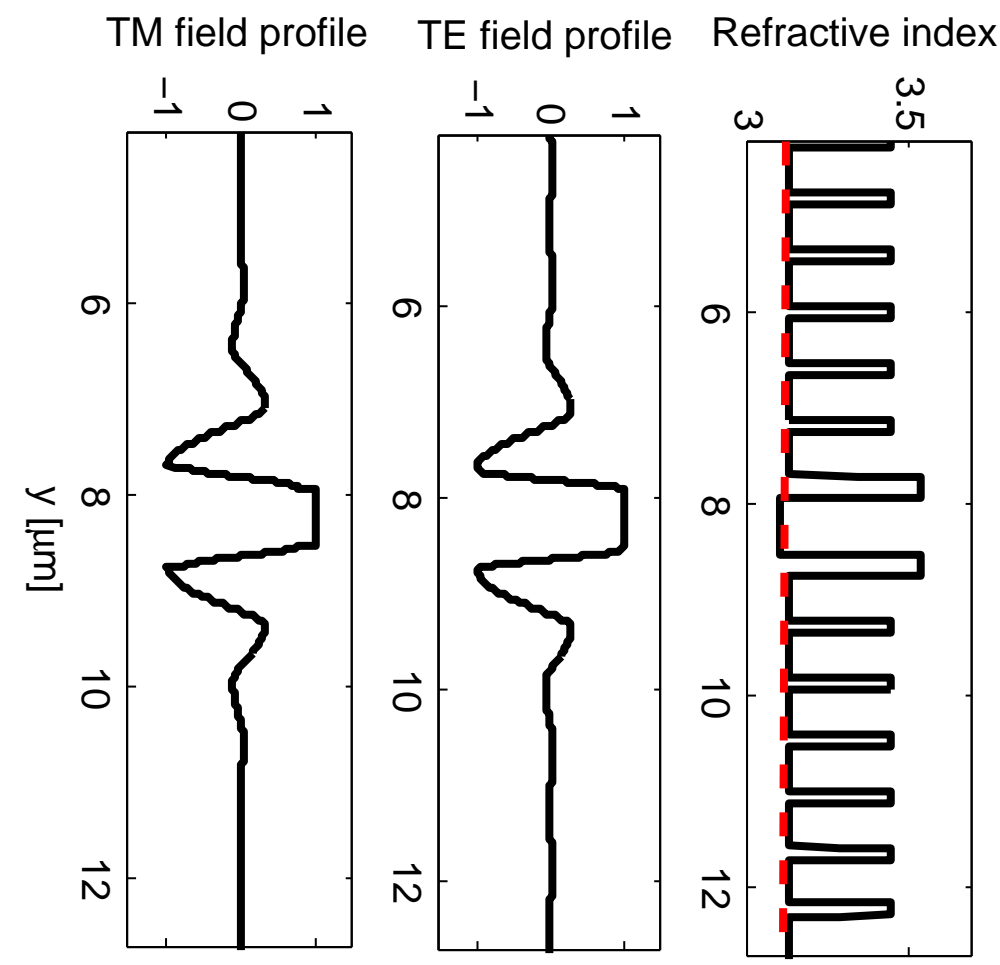

Figure 2.8 - TE (left) and TM (middle) polarizations of an interface mode at the boundary between two semi-infinite periodic layered media with cap layers separated by a homogenous medium layer; the index profile is also shown (right), the red dashed line designates the mode effective index.

The band diagrams of TE and TM modes in such a structure are shown in Fig. 2.9. TIR and interface modes coexist in this structure. The coupled interface modes appear below the core light line which agrees with the their evanescence in the core. TIR modes appear outside the multi-layer bandgap and below the light line of the core and the low index layer in the cladding; therefore, they are evanescent in the core and in the low index cladding layers and delocalized in the cap layer and the high index cladding layer. Bragg modes also appear in the light cone of the core. Their effective index is smaller than that of the core and are hence delocalized in the core but evanescent in the stacks; in this particular case there are odd modes. Fig. 2.10 shows the field distribution of the TIR mode that exists alongside the interface modes in this structure. 

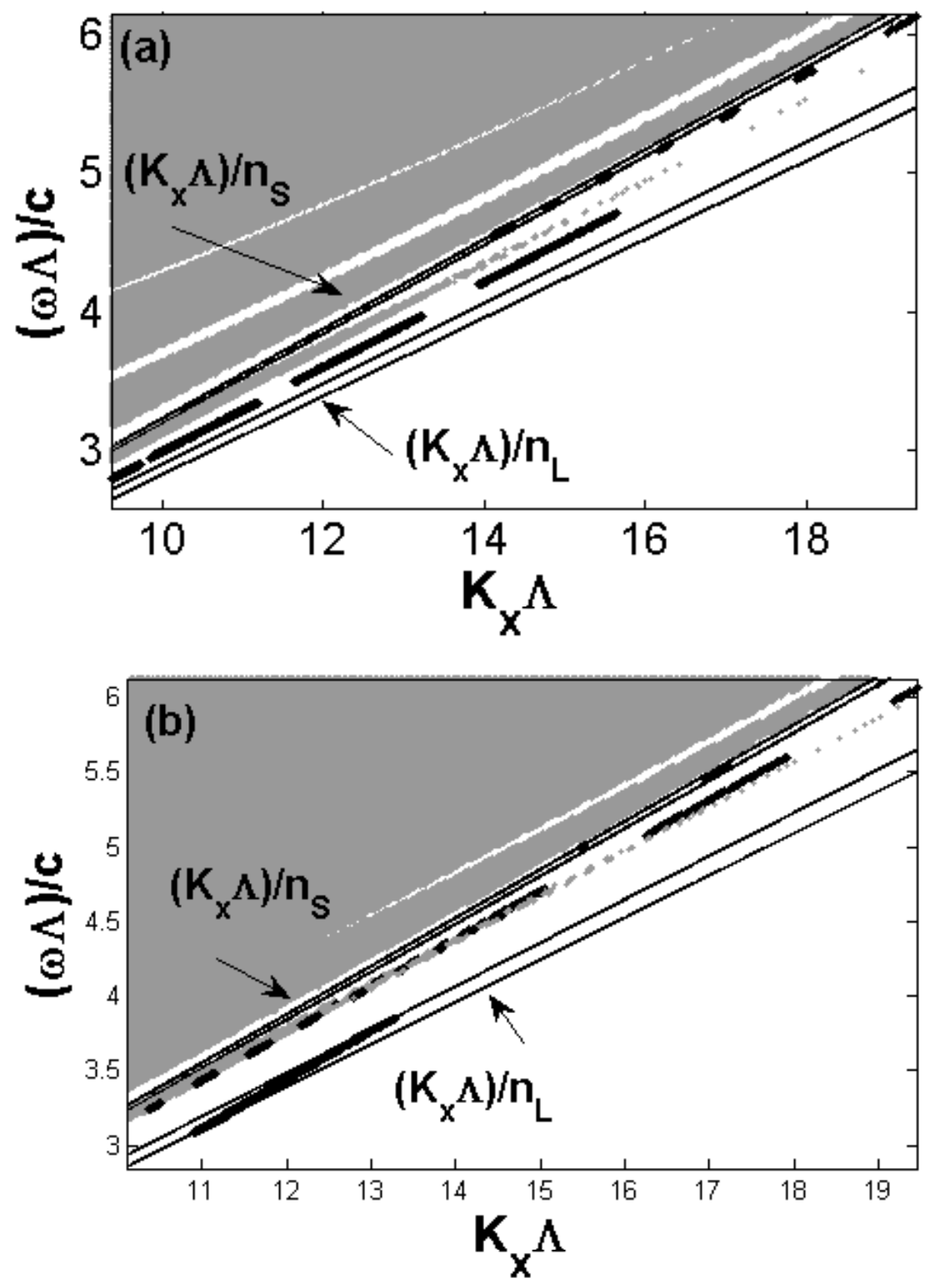

Figure 2.9 - TE (top) and TM (bottom) band diagrams of guided states at the boundary between two semi-infinite periodic layered media with cap layers separated by a thick homogenous medium layer. 


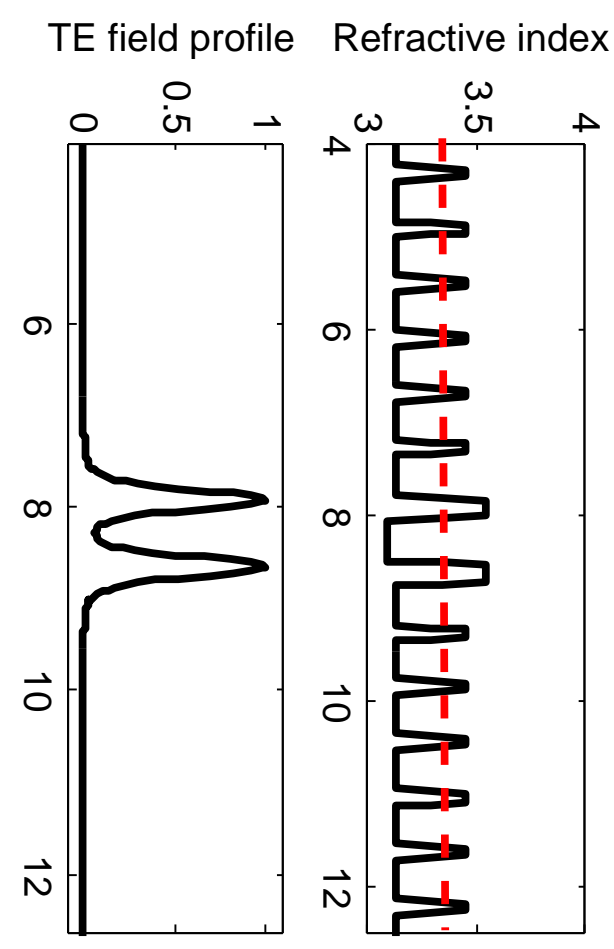

Figure 2.10 - Mode profile (left) of the TIR mode in the structure consisting of two semiinfinite periodic layered media with cap layers separated by a thin homogeneous medium; the index profile is also shown (right), the red dashed line designates the mode effective index.

\subsection{Coupled Interface Modes for Utilization in Non- linear Interaction}

The dispersion relations for these coupled interface modes are very similar to those of Bragg modes investigated in [9]. The main difference arises from the fact that the range for the interface mode effective index is different from that of Bragg modes.

In Bragg modes, the field is non-evanescent in the core as well as the claddings. This constraints the range of the effective mode index to

$$
0<\mathrm{n}_{\mathrm{eff}, \mathrm{BRW}}<\mathrm{n}_{\min }
$$

where $\mathrm{n}_{\min }$ is the minimum refractive index of the structure including the core, the cover 
and the substrate.

However, in the interface modes of case of section 1.4 discussed above, the field is evanescent in the core and hence the mode effective index is limited by the core refractive index

$$
\mathrm{n}_{\text {core }}<\mathrm{n}_{\text {eff,IM }}<\mathrm{n}_{\text {min }}
$$

where $\mathrm{n}_{\min }$ this time, is the minimum refractive index of the cladding layers and the cover and substrate.

This difference causes the original definition of the core wave vector to be imaginary. That is why the real equivalent of the wave vector, $\mathrm{k}_{\mathrm{c}, \text { real }}$, is defined, $\mathrm{k}_{\mathrm{c}}=i \mathrm{k}_{\mathrm{c}, \text { real }}$ and used throughout the calculations.

In order to achieve phase matching between an interface mode and one that is guided through Total Internal Reflection in a second order nonlinear process, it is required that a high refractive index layer be surrounded by lower "effective" index media. Moreover, efficient overlap between the profiles of the interacting waves entails utilization of even modes for the interacting waves. Consequently, the boundary between a high index cap layer followed by a stack that consists of periods of low and high index materials and a homogenous medium would make a good candidate for supporting both TIR and interface modes.

The coupled interface modes of Fig. 2.8 can further be brought together by shrinking the core size as seen in Fig. 2.11. As discussed in [14], bringing together two such structures close enough such that the two interface modes are coupled, provides symmetric mode profiles with enhanced mode overlap. This eliminates the odd modes and only the even modes survive. The terms "thin" and "thick" will be used to refer to this elimination of the odd modes. Eliminating the odd modes further assists with fundamental mode discrimination which is very useful in exciting the right mode in applications such as lasers [30]. Another advantage of "thin" core layers versus the "thick" ones is that 
the mode profile of the closely coupled modes resembles and overlaps better with that of TIR or a Bragg mode. Overlap between the interacting modes, as shall be discussed in Chapter 3, is crutial for efficient frequency mixing. The TE and TM band diagrams for these thin core coupled interface modes are shown in Fig. 2.12. They are very similar to the band diagrams of the case with a thick core layer inside as expected.

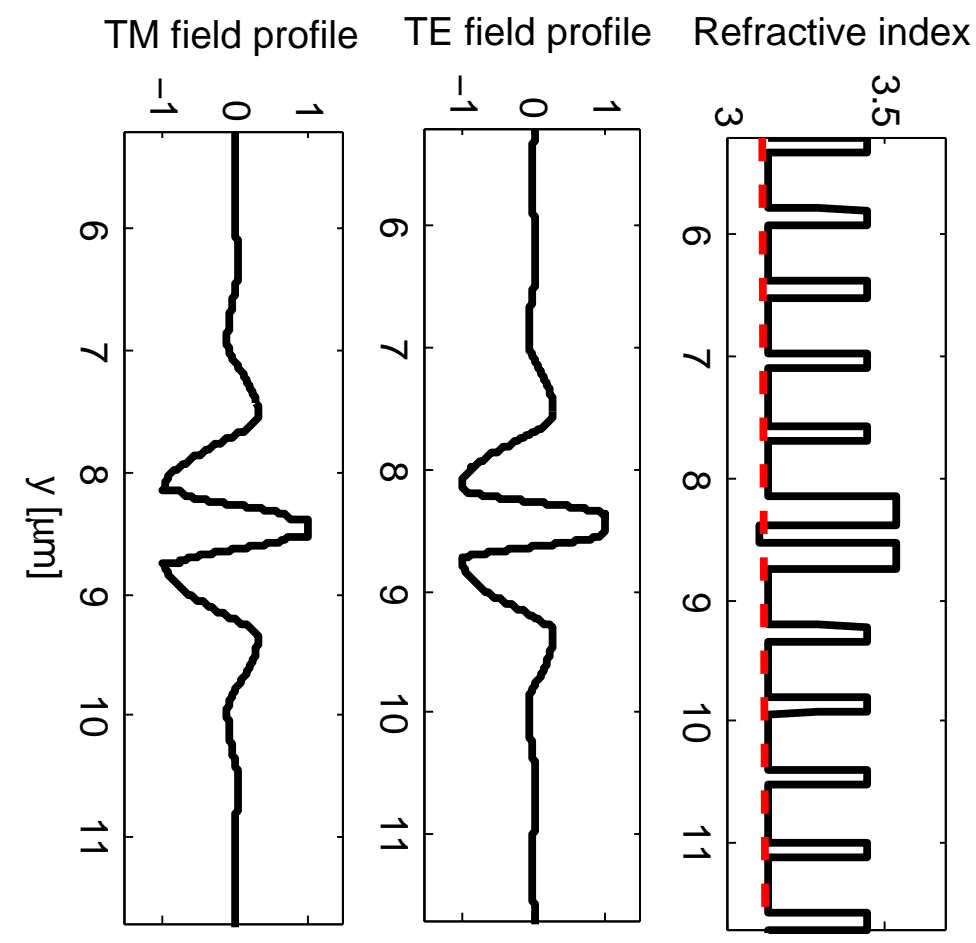

Figure 2.11 - TE (left) and TM (middle) mode profiles of an interface mode at the boundary between two semi-infinite periodic layered media with cap layers separated by a thin homogenous medium layer; the index profile is also shown (right), the red dashed line designates the mode effective index.

Like the structure of section 2.2, these conjugated capped stacks provide the potential to be used in laser diodes. In much the same way that the structure in section 2.3 provided more tuning parameters and hence more flexible design than that in section 2.1, the conjugated capped stacks offer greater degrees of freedom over the conventional noncapped counterpart. 

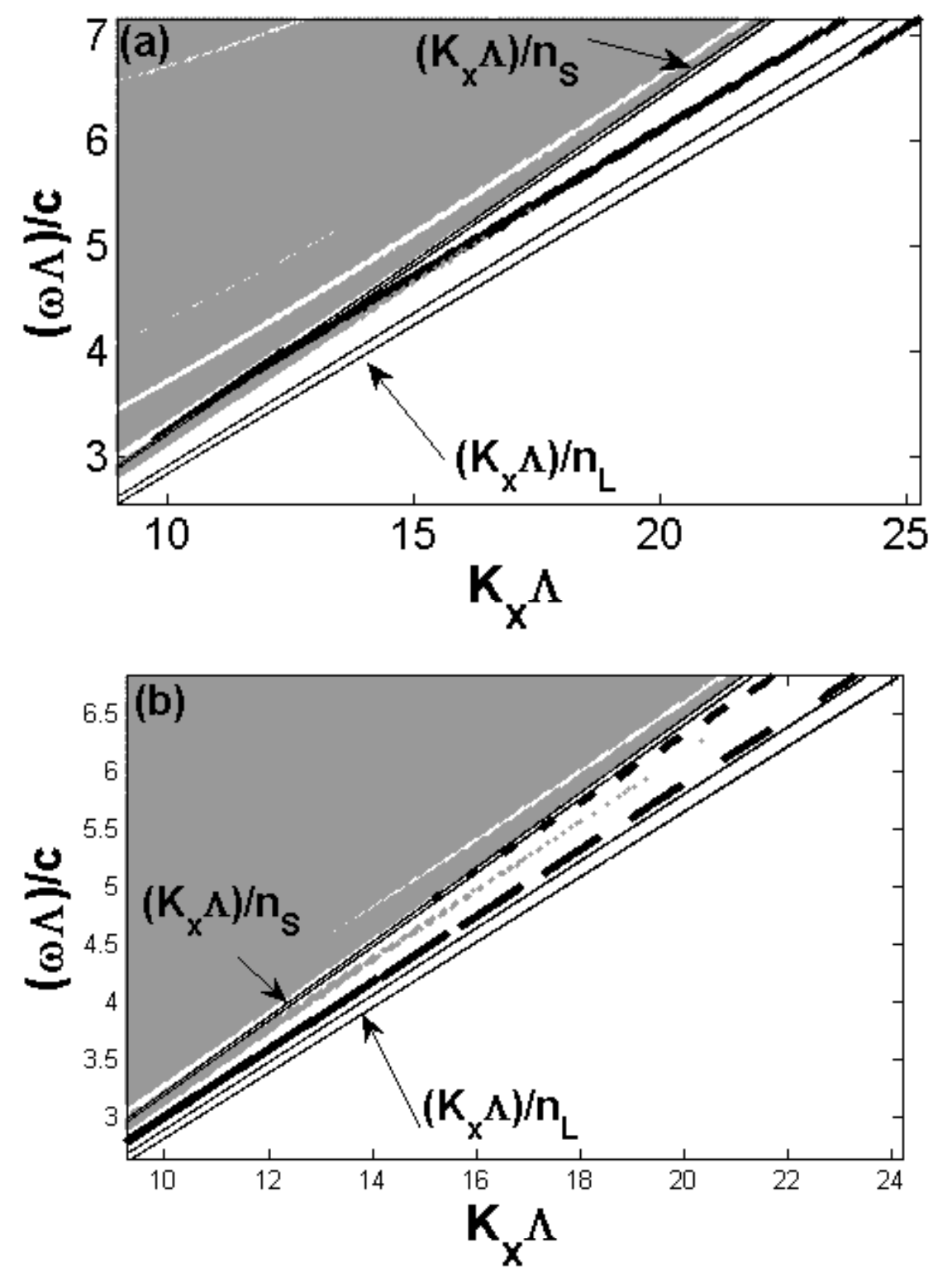

Figure 2.12 - TE (top) and TM (bottom) band diagrams of guided states at the boundary between two semi-infinite periodic layered media with cap layers separated by a thin homogenous medium layer. 
Also, similar to the Bragg modes in BRWs, these interface modes can be phase matched to other modes that exist within the same structure for nonlinear optical processes. The next chapter discusses this potential of the coupled interface modes and builds a foundation for experimentally demonstrating such a phenomenon. 


\section{Chapter 3}

\section{Phase Matching and Optimization of Second Order Nonlinear Interaction Using Interface Modes}

A periodic structure such as the one discussed in section 2.4, can benefit second order nonlinear processes and in particular, Second Harmonic Generation. Bragg Reflection Waveguides have much in the same way been utilized for nonlinear optical interactions $[9$, 10, 29] as discussed in Chapter 1. Designing the structure to accommodate two different types of modes and optimizing the design such that these two modes are phase matched is critical in achieving nonlinear interaction. Additionally, the structures can be further optimized to accommodate low loss, high efficiency SHG. This chapter discusses Second Harmonic Generation using a periodic layered medium and the phase matching scheme and the parameters involved in this nonlinear process optimization. 


\subsection{Phase-Matching the SHG process Using Inter- face Modes}

In the first Chapter of this work, a review of Second Harmonic Generation using Bragg Reflection waveguides was given. Later, in the second Chapter the dispersion relations for Coupled interface modes were provided. Utilizing the same method used on BRWs, one can achieve SHG in a system containing a fundamental TIR mode and a second harmonic coupled interface mode.

An essential condition in efficient nonlinear interaction as discussed in the first chapter is that the phases of the interacting waves be matched according to the process. In SHG, several phase matching types can be explored. The two discussed within this work, are known as type I and type II phase matching. In type I phase matching, the k-vector of the TE fundamental wave is matched to that of the TM second harmonic wave. In type II phase matching, the k-vectors of the TE+TM fundamental waves are matched to the TE second harmonic wave. The guiding equations for these two conditions are,

$$
\begin{gathered}
\text { TypeI : } n_{e f f, T E, \omega}=n_{\text {eff }, T M, 2 \omega} \\
\text { TypeII : } 0.5\left(n_{e f f, T E, \omega}+n_{e f f, T M, \omega}\right)=n_{e f f, T E, 2 \omega}
\end{gathered}
$$

In order to match the phases of the modes, a generic design procedure can be carried out to produce a structure in which both modes propagate with the same effective refractive index. In deriving the equations of section II, no assumptions were made about the range of the mode index; therefore, the TIR mode can be modeled using the same equations employed for interface mode characterization. In reality, the TIR mode index sits somewhere between the core index and the cladding indices and is hence confined within the core mostly through total internal reflection.

Using the design equations in section 2.4, given the core thickness and structure refractive indices, cap and cladding layer thicknesses can be determined for a QtW struc- 
ture that supports an interface mode. The method described here summarizes the design technique used to phase match the two modes.

- For a set of effective refractive indices lying in the range of equation 2.32, the following steps are performed:

1. The $\mathrm{k}$ vectors for the cladding layers are calculated according to $k_{i x}=k_{0} \sqrt{n_{i}{ }^{2}-n_{\text {eff }}^{2}}$ where $i=1,2$, cap.

2. The cladding layer thicknesses are obtained assuming the quarter-wave condition

3. The dispersion relations in Eq. 2.29-2.30 are used to calculate the corresponding cap layer thickness for both TE and TM interface modes

4. With the index and thickness sets in hand, the TE and TM TIR modes at frequency $\omega$ are sought and the mode indices are obtained

- In the case of Type I phase matching, the set of TM Bragg mode indices at $2 \omega$ are plotted along with the corresponding TE TIR mode indices at $\omega$ and the point of intersection is refined until a structure is found where the effective mode indices of the two waves are equal.

- In the case of Type II phase matching, the set of TE Bragg mode indices at $2 \omega$ are plotted along with the average of the TE and TM TIR mode indices at $\omega$ and the point of intersection is refined.

- This point of intersection where the effective mode indices are equal is the phase matching point, and the device characterization would be carried out at the corresponding wavelength.

Fig. 3.1 shows a schematic of the mode profiles for both the TIR and the interface modes for a type II phase-matched structure. The TIR mode effective index is expected to range from the cap layer index to the core index. 


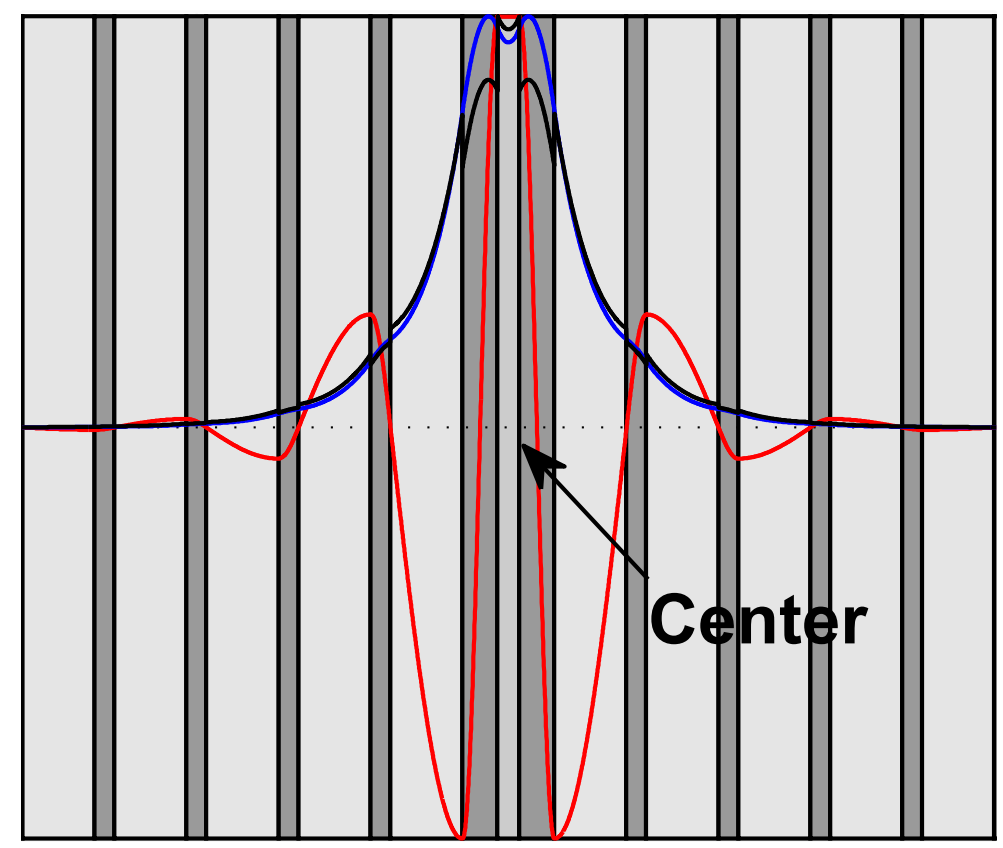

Figure 3.1 - A schematic of the profiles for TIR TE (blue) and TM (black) modes and TE coupled interface mode (red) for type II phase matching.

\subsection{Mode Properties for Optimum Nonlinear Inter- action}

Several process parameters can be tailored in order to achieve an efficient second order nonlinear conversion. Nonlinear Conversion efficiency, Group Velocity Dispersion ( GVD) and Group Velocity Mismatch ( GVM ) are amongst the most important ones.

Here, the effects of structure properties on each of these parameters and the importance of optimizing them is discussed.

\subsubsection{Nonlinear Conversion Efficiency}

Nonlinear Coupling Efficiency defines how effectively energy is coupled between the fundamental and second harmonic waves. This efficiency can thus be defined as 


$$
\eta=\frac{1}{t_{S H G}^{e f f} n_{\text {eff }}^{3}}=\frac{1}{n_{\text {eff }}^{3}} \frac{\left[\int_{-8}^{8}\left[E^{2 \omega}(x)\right] d_{\text {norm }}\left[E^{\omega}(x)\right]^{2} d x\right]^{2}}{\left.\left[E^{\omega}(x)\right]^{2} d x\right]^{2}\left[\int_{-8}^{8}\left[E^{2 \omega}(x)\right]^{2} d x\right]}
$$

where $t_{S H G}^{e f f}$ is the effective SH width and $d_{\text {norm }}=\frac{d}{d_{\max }} \quad$ is the normalized quadratic nonlinear optical coefficient of AlGaAs which varies with $x$, since it is dependant on the aluminium concentration. The calculation of this nonlinear optical coefficient was based on a study by Ohashi et al. [36], that used reflected second harmonics to experimentally determine the dependence of the magnitude of $d$ on the aluminium concentration in AlGaAs.

The propagation length also plays a role in the efficiency; however this effect is counteracted by the propagation losses and hence the normalized efficiency is still a relatively informative figure of merit. A more useful parameter to study for device efficiency comparison is the conversion efficiency and depends on the spatial overlap between the polarization density at the second harmonic defined as $P^{2 w}=\varepsilon_{0} d(x)\left[E^{w}(x)\right]^{2}$ and the mode at $2 \omega$.

The normalized conversion efficiency is hence defined as,

$$
\eta_{\text {norm conv }}=\frac{P_{2 \omega}}{P_{\omega}^{2}} \frac{1}{L^{2}}=\frac{2 \omega^{2} d_{e f f}^{2}}{n_{\text {eff }}^{3} t_{S H G}^{e f f}}\left(\frac{\mu_{0}}{\epsilon_{0}}\right)^{\frac{3}{2}}
$$

where $P_{2 \omega}$ and $P_{\omega}$ are the internal second harmonic and pump powers and $\mathrm{L}$ is the waveguide length.

The behavior pattern for the efficiency as a function of the aluminium concentrations of all layers as well as the core thickness was studied. The most dominant changes in the conversion efficiency leading to maximized values occurred in the parameter space of $\left(t_{c}, x_{2}\right)$; therefore $\eta_{\text {normconv }}$ was plotted versus variations of core thickness, as well as aluminium concentrations of layers. Fig. 3.2 further depicts a contour plot of the efficiency as a function of core thickness and second layer aluminium concentration for both types of phase matching. Over a range of $100 \mathrm{~nm}$ core thickness, and the aluminium 
fraction variation of the second cladding layer, the normalized conversion efficiency for both type I and type II phase matching techniques has been maximized. The choice of parameter ranges was based on the capability of the structure to support phase matched modes. The aluminium concentrations were also upper-limited due to oxidation and lower-limited due to two-photon absorption [37].

For a fixed value of core thickness, the efficiency increases as the aluminium concentration of the second layer increases as expected due to the increased refractive index contrast between the layers which confines the SH mode further, increasing the coupling efficiency between the fundamental and second harmonic waves. For a fixed value of $x_{2}$ however, there exists a local maximum in the value taken by the conversion efficiency. At smaller core thickness values, the first negative lobes of the $\mathrm{SH}$ mode is so large that most of the mode energy is concentrated in them, away from the three center layers of the structure where overlap with TIR is greatest. At larger core thickness values, especially for lower $x_{2}$, the $\mathrm{SH}$ wave starts to get less and less confined. The addition of lobes including those with the opposite sign to that of the TIR is a factor that lowers the overlap of the two waves and hence the conversion efficiency. Therefore, there's a point at which these two opposite effects give rise to an optimum core thickness.

\subsubsection{Group Velocity Mismatch}

Another parameter that plays an important role in increasing the nonlinear interaction length and hence the process efficiency depends on the temporal walk off between the first and second harmonic pulses. Group Velocity Mismatch (GVM) is a measure of this temporal separation and minimizing this parameter leads to a more efficient nonlinear interaction between the two pulses $[38,39]$. GVM can quantitatively be defined as

$$
G V M=\left|\frac{1}{v_{g}^{(w)}}-\frac{1}{v_{g}^{(2 w)}}\right|=\frac{1}{c}\left|n_{g}^{(w)}-n_{g}^{(2 w)}\right|
$$

where $n_{g}^{(w)}$ and $n_{g}^{(2 w)}$ are the group indices of the two interacting pulses. 

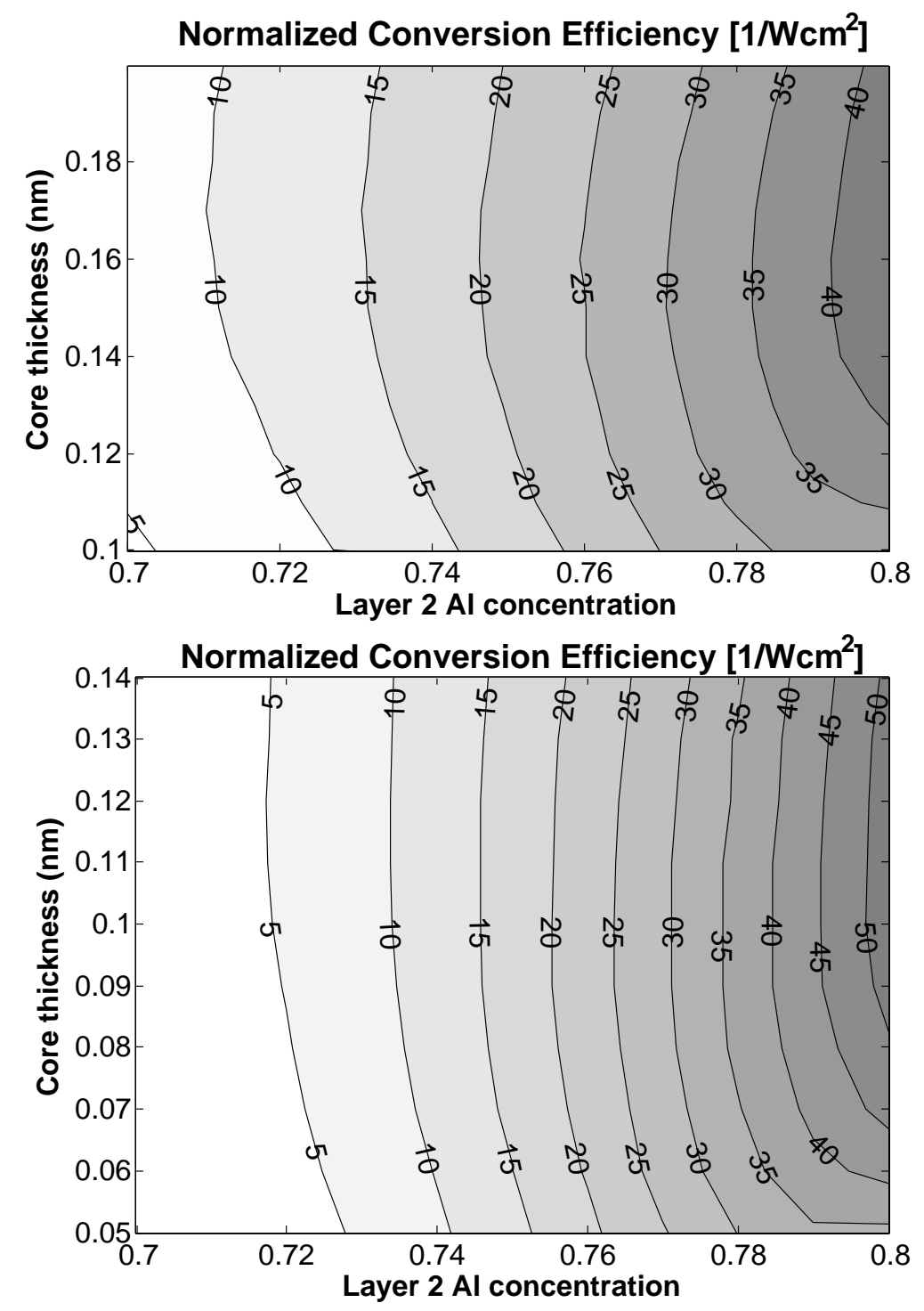

Figure 3.2 - Normalized coupling efficiency for type I (top) and type II (bottom) $P M$ as a function of core thickness and $x_{2}$; the structure parameters for type $I$ are $(x 1, x m, x c)=(0.3,0.2,0.85)$ maximum happens at $\left(t_{c}, x_{2}\right)=(160 \mathrm{~nm}, 0.8)$ and has a value of $0.41 \times 10^{-4}\left[1 / W \mathrm{~cm}^{2}\right]$. The structure parameters for type II are $(x 1, x m, x c)=$ $(0.3,0.2,0.85)$ maximum efficiency happens at $\left(t_{c}, x_{2}\right)=(140 \mathrm{~nm}, 0.8)$ and has a value of $0.515 \times 10^{-4} \quad\left[1 / W_{c m}^{2}\right]$. 
A contour plot of the GVM is shown in Fig. 3.3. For aluminium fraction variations of the cap and the core layers, the group velocity mismatch between the fundamental and second harmonic waves is calculated for type I phase matching. The minimum GVM value occurs at $\left(\mathrm{x}_{\mathrm{c}}, \mathrm{x}_{\mathrm{m}}\right)=(0.8,0.35)$ and has a value of $2.6354\left(\frac{\mathrm{ps}}{\mathrm{mm}}\right)$.

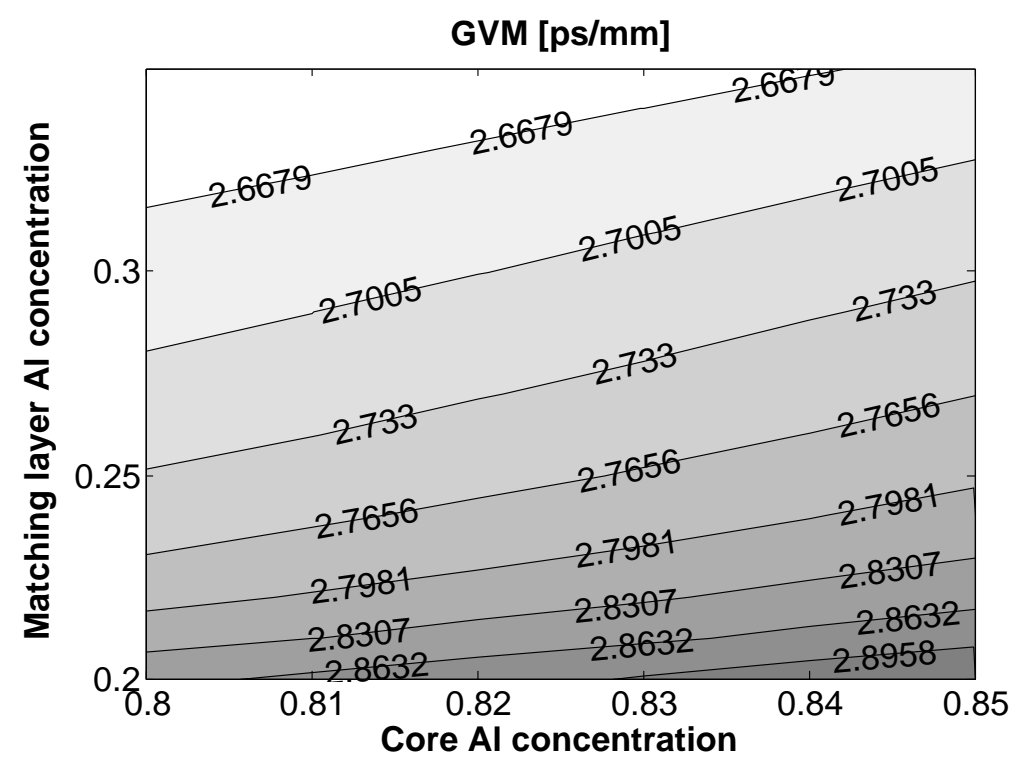

Figure 3.3 - GVM as a function of cap and core layer aluminium concentrations; the structure parameters are $(x 1, x 2, t c, t m)=(0.4,0.55,310 n m, 286.9 n m)$. Minimum GVM happens at $\left(x_{c}, x_{m}\right)=(0.8,0.35)$ and has a value of $2.6354\left(\frac{\mathrm{ps}}{\mathrm{mm}}\right)$.

The parameter space was again based on the limitations imposed by aluminium oxidation and two-photon absorption and the phase matching requirement.

\subsubsection{Group Velocity Dispersion}

One last parameter that becomes important particularly in ultra short pulse applications, is Group Velocity Dispersion (GVD). Apart from the undesirable pulse distortion that results from group velocity dispersion, a reduction in the peak pulse power due to GVD will further reduce the conversion efficiency; hence, minimizing the effects of this parameters is beneficial to the nonlinear process.

Group Velocity Dispersion is defined as 


$$
G V D=\frac{\partial^{2} \beta}{\partial \omega^{2}}=\frac{2}{c} \frac{\partial n_{e f f}}{\partial \omega}+\frac{\omega}{c} \frac{\partial^{2} n_{e f f}}{\partial \omega^{2}}
$$

where $\omega$ is the frequency at which the dispersion is happening, and c is the free space speed of light.

Fig. 3.4 illustrates the dependence of GVD on $\mathrm{Al}$ concentrations for the TIR first harmonic pulse. Fig. 6.4 illustrates this dependence for the IM second harmonic pulse.

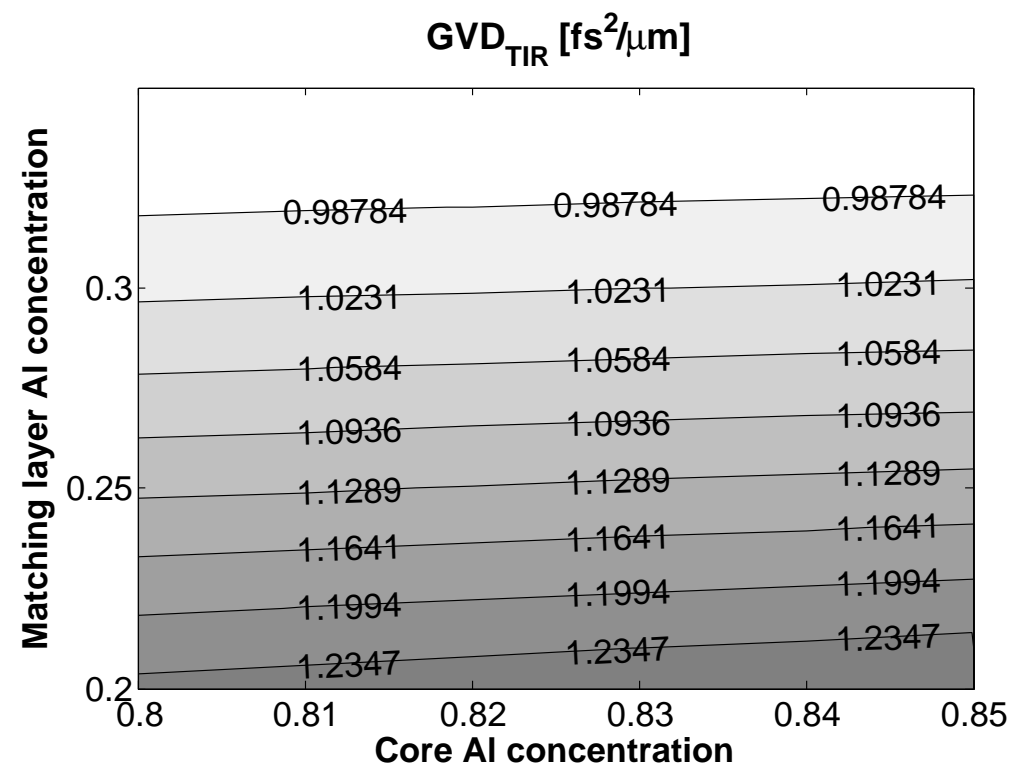

Figure 3.4 - TIR GVD as a function of cap and core layer aluminium concentrations; the structure parameters are $\left(x_{1}, x_{2}, t_{c}, t_{c a p}\right)=(0.4,0.55,310 \mathrm{~nm}, 286.9 \mathrm{~nm})$. Minimum $G V D$ happens at $\left(x_{c}, x_{m}\right)=(0.8,0.35)$ and has a value of $0.95258\left(\frac{f s^{2}}{\mu m}\right)$.

\subsection{Performance of Coupled Interface Modes}

The main motivation behind the development of coupled interface modes was to establish an additional means of achieving phase matching in a perdiodic layered medium and to gain extra degrees of freedom in tailoring the mode linear and nonlinear properties.

Since previous studies on BRWs and matching layer-enghanced BRWs have been mainly focused on generating the second harmonic of a pump at $1550 \mathrm{~nm}$, a similar set 


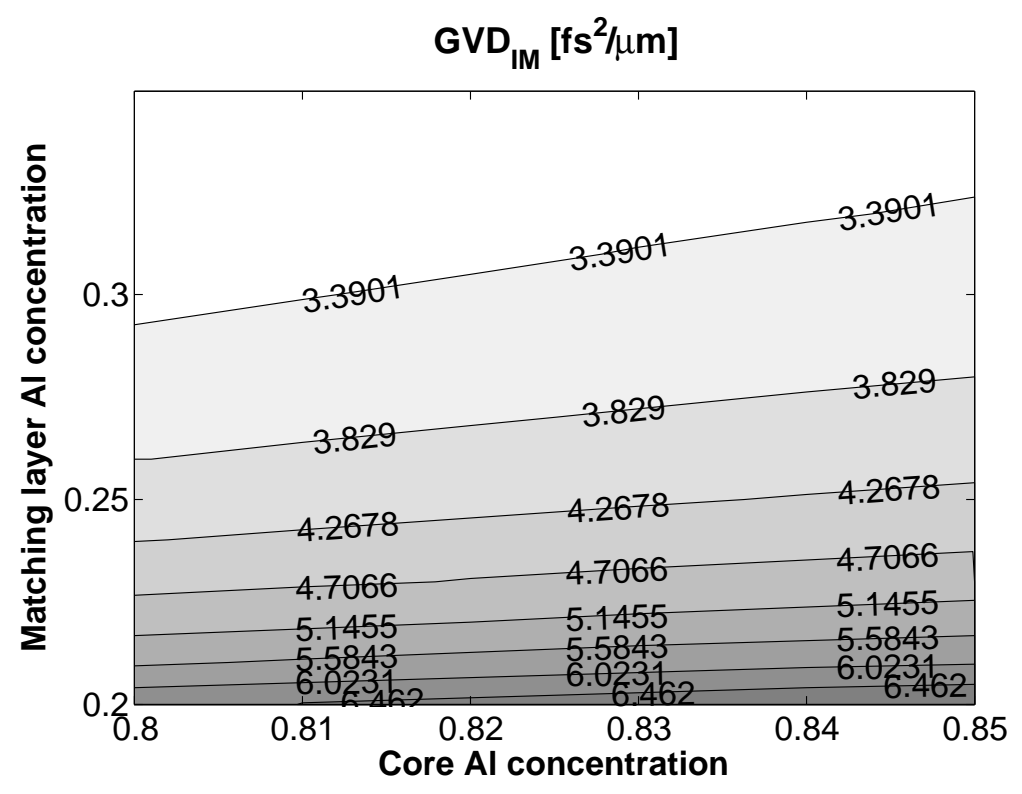

Figure 3.5 - CIM GVD as a function of cap and core layer aluminium concentrations; the structure parameters are $\left(x_{1}, x_{2}, t_{c}, t_{\text {cap }}\right)=(0.4,0.55,310 \mathrm{~nm}, 286.9 \mathrm{~nm})$. Minimum GVD happens at $\left(x_{c}, x_{m}\right)=(0.8,0.35)$ and has a value of $2.9513\left(\frac{f s^{2}}{\mu m}\right)$.

up will be used as a reference for comparison purposes. The performance of coupled interface modes can be examined against those of conventional and matching layer-enhanced Bragg reflection waveguides via analyzing device performance parameters such as those mentioned in the last section.

The tables below list the values of the above critical parameters in a particular interface mode waveguide design and compares them to those found in [9] and [29]. In the comparison of table 3.1,each design aims at maximizing the conversion efficiency. Table 3.2 compares the Interface mode structure design to the matching-layer enhanced design where GVM has been maximized. Finally, table 3.3 draws a similar comparison where the second harmonic GVD has been maximized.

All three types of modes listed in the tables belong to structures with quarter-wave stacks. In the conventional BRWs, this quarter-wave condition imposes a limitation on the thickness of the core. Moreover, higher conversion efficiency values involve a choice of cladding materials that corresponds to greater temporal walk-off between the harmonics 
Table 3.1 - Comparison of Mode Parameters for Different Multilayer Designs

\begin{tabular}{|l|l|l|l|l|}
\hline Design & $\eta_{\text {norm conv }}\left[1 / \mathrm{Wcm}^{2}\right]$ & GVM $[\mathrm{ps} / \mathrm{mm}]$ & $\begin{array}{l}\mathrm{GVD}\left[\mathrm{fs}^{2} / \mu m\right] \\
\mathrm{SH}\end{array}$ & $\begin{array}{l}\mathrm{GVD}\left[\mathrm{fs}^{2} / \mu m\right] \\
\mathrm{FH}\end{array}$ \\
\hline BRW & $0.638 \times 10^{-4}$ & 2.3674 & 1909 & 763 \\
\hline ML & $2.15 \times 10^{-4}$ & 2.4058 & 6.6349 & 1.4398 \\
\hline C-IM & $0.410 \times 10^{-4}$ & 2.1483 & 9.6646 & 1.3674 \\
\hline
\end{tabular}
been the target; BRW= Bragg Reflection Waveguide supporting a Bragg mode, $M L=$ Matching Layer enhanced Bragg reflection waveguides supporting a Bragg mode, C-IM= Multilayer supporting Coupled Interface Modes.

Table 3.2 - Comparison of Mode Parameters for Different Multilayer Designs

\begin{tabular}{|l|l|l|l|l|}
\hline Design & $\eta_{\text {norm conv }}\left[1 / \mathrm{Wcm}^{2}\right]$ & $\mathrm{GVM}[\mathrm{ps} / \mathrm{mm}]$ & $\begin{array}{l}\mathrm{GVD}\left[\mathrm{fs}^{2} / \mu m\right] \\
\mathrm{SH}\end{array}$ & $\begin{array}{l}\mathrm{GVD}\left[\mathrm{fs}^{2} / \mu m\right] \\
\text { FH }\end{array}$ \\
\hline $\mathrm{ML}$ & $0.04532 \times 10^{-4}$ & 1.73 & 2.8749 & 1.2616 \\
\hline $\mathrm{C}-\mathrm{IM}$ & $0.0028 \times 10^{-4}$ & 2.6354 & 2.9513 & 0.9526 \\
\hline
\end{tabular}

the target; $M L=$ Matching Layer enhanced Bragg reflection waveguides supporting a Bragg mode, $C$-IM= Multilayer supporting Coupled Interface Modes.

and hence greater GVM [40]. While operation away from the quarter-wave point appears to be a favorable alternative in order to avoid these limitations, the lack of closed-form 
Table 3.3 - Comparison of Mode Parameters for Different Multilayer Designs

\begin{tabular}{|l|l|l|l|l|}
\hline Design & $\eta_{\text {norm conv }}\left[1 / W \mathrm{~cm}^{2}\right]$ & GVM $[p s / m m]$ & $\begin{array}{l}\mathrm{GVD}\left[f s^{2} / \mu m\right] \\
\mathrm{SH}\end{array}$ & $\begin{array}{l}\mathrm{GVD}\left[\mathrm{fs}^{2} / \mu m\right] \\
\mathrm{FH}\end{array}$ \\
\hline $\mathrm{ML}$ & $0.0145 \times 10^{-4}$ & 1.9259 & 2.2125 & 1.1704 \\
\hline $\mathrm{C}-\mathrm{IM}$ & $0.0028 \times 10^{-4}$ & 2.6354 & 2.9513 & 0.9526 \\
\hline
\end{tabular}

All listed parameters are designed for type I phase matching where the second harmonic mode GVD optimization has been the target; $M L=$ Matching Layer Enhanced Bragg Reflection Waveguides supporting a Bragg mode, C-IM = Multilayer supporting Coupled Interface Modes.

expressions for analytically design a structure and optimize it, make the quarter-wave point an appealing operation region.

In the conventional Bragg reflection waveguide designs [29], there is a trade-off between the conversion efficiency and group velocity dispersion of the second harmonic in particular and hence the design of these waveguides is application oriented as to optimize the right parameters. While application is also considered in the design of CIMs, these modes also benefit from the fact that large conversion efficiency values are not necessarily available only at the cost of greater group velocity dispersion.

GVD values are very close to those in [9]. The stacks can be tailored even further by means of optimizing the refractive index and thickness values as well as introducing additional design parameters such as defect layers or chirp in the claddings. Moreover, non-degenerate three wave mixing processes such as sum and difference frequency generation are potentially achievable through utilizing all the possible modes accommodated by the capped multilayer structure. 


\section{Chapter 4}

\section{Wafer Design for Efficient Second Harmonic Generation}

Once the theoretical tools to explain and predict the behaviour of Coupled Interface Modes have been developed, it is possible to design structures that accommodate Second Harmonic Generation using these modes. In this chapter, a new design for type II phase matched CIMs is be provided. The main focus of the design is maximizing the normalized nonlinear conversion efficiency according to the contour plots of Chapter 3. The refractive indices of the simulated structure are those of $\mathrm{Al}_{x} \mathrm{Ga}_{1-x} \mathrm{As}$ at the operating wavelengths. Later, Type I phase matching within the same structure is sought and some of the process parameters are theoretically predicted.

\subsection{Design Considerations}

In designing a practical structure, several considerations are taken into account; these considerations are breifly listed below:

1. The refractive index values available to the design are very limited. The $x$ aluminium fraction in AlGaAs can in theory range from 0 (GaAs) to 1 (AlAs); how- 
ever, in practice, there are upper and lower bounds to the values $x$ can take. The lower bound originates from the effects of two-photon absorption that will interfere with the process for aluminium concentration values below 0.18 [37]. Setting aside a safety margin for this value, the minimum $x$ available for a practical design is about 0.2 .

2. The upper bound for the refractive index is set due to fast oxidization of very high Al concentration AlGaAs. Therefore, an upper limit of $x=0.85$ has been set.

3. Molecular Beam Epitaxy is used to grow the layers of AlGaAs on a GaAs substrate. Lattice matching is critical in this method and so the growth of two adjacent layers with a great contrast of $\mathrm{Al}$ concentration is prone to surface defects and hence deteriorated device performance. The thicker such two layers become, the greater the risk of growth defects would be.

4. The overall thickness of the grown layers should not exceed 8-9 $\mu \mathrm{m}$. This limits the layer thickness as well as the number of periods added to the claddings.

5. Observation of Second harmonic generation for both type I and type II within the same structure requires operations in a wavelength range that is deliverable by the available light sources; often, type I and type II phase matched processes happen in wavelengths are far apart and hence both need to be designed within the available range.

It is also worth noting that greater refractive index contrast between the layers and more periods in the claddings are both factors that decrease the losses, and confine the modes, giving rise to greater efficiency. With the above limitations and trade offs in mind, the structures can be designed, fabricated and tested for confirmation of the potential of CIMs for second order nonlinear processes. 


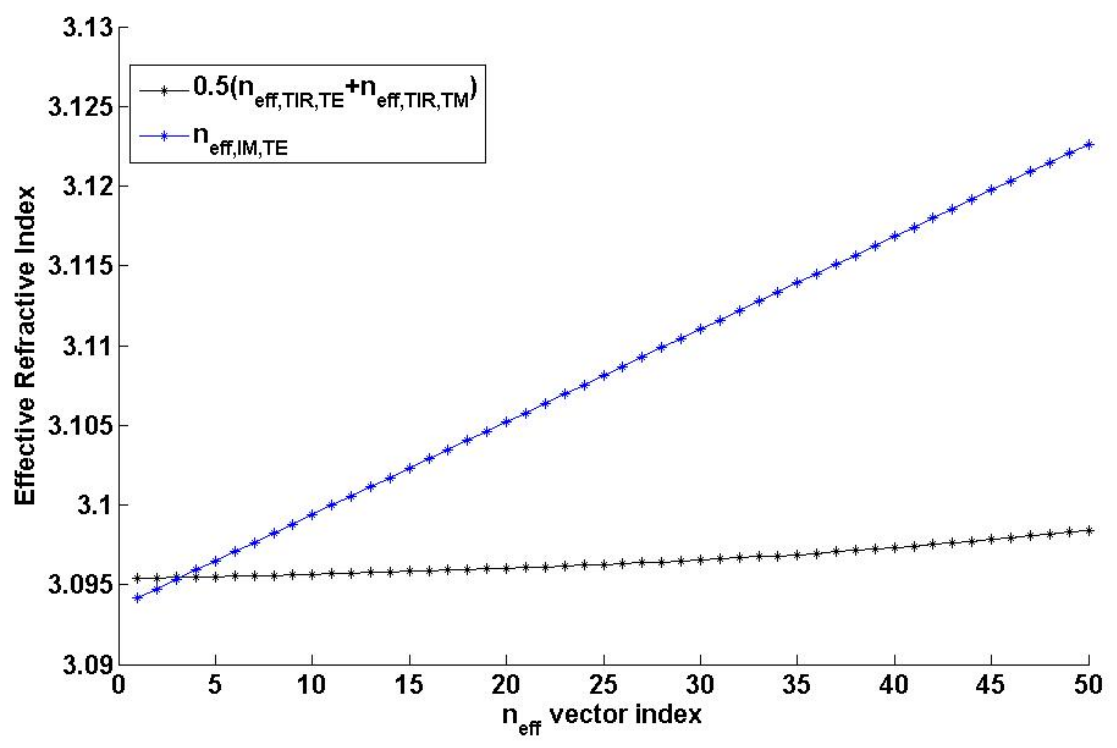

Figure 4.1 - The intersection of the TE second harmonic wave refractive index and the average of TE and TM indices of the fundamental waves for type II phase matching.

\subsection{Structure Design of Coupled Interface Modes for Type II phase matching}

Refractive index values are chosen for all the layers according to the contour plots in Chapter 3. The procedure outlined in Section 3.2 is carried out for type II phase matching to arrive refractive index that corresponds to the point of phase matching. The fundamental operating wavelength is chosen to be $\lambda=1540 \mathrm{~nm}$ which corresponds to a second harmonic wavelength of $\lambda=770 \mathrm{~nm}$. Fig. 4.1 shows the intersection between the refractive index of the TE second harmonic wave and the average of TE and TM indices of the fundamental waves.

The top plot in Fig. 4.2 shows the refractive index profiles of the structure at the two fundamental and second harmonic wavelengths. The effective index common to the two profiles is shown with a dashed line. This is the same point of intersection in Fig. 4.1. The mode profiles of all of these three waves are also shown in Fig. 4.2, superimposed on the structure schematic. The plot at the bottom of Fig. 4.2, demonstrates the phase 

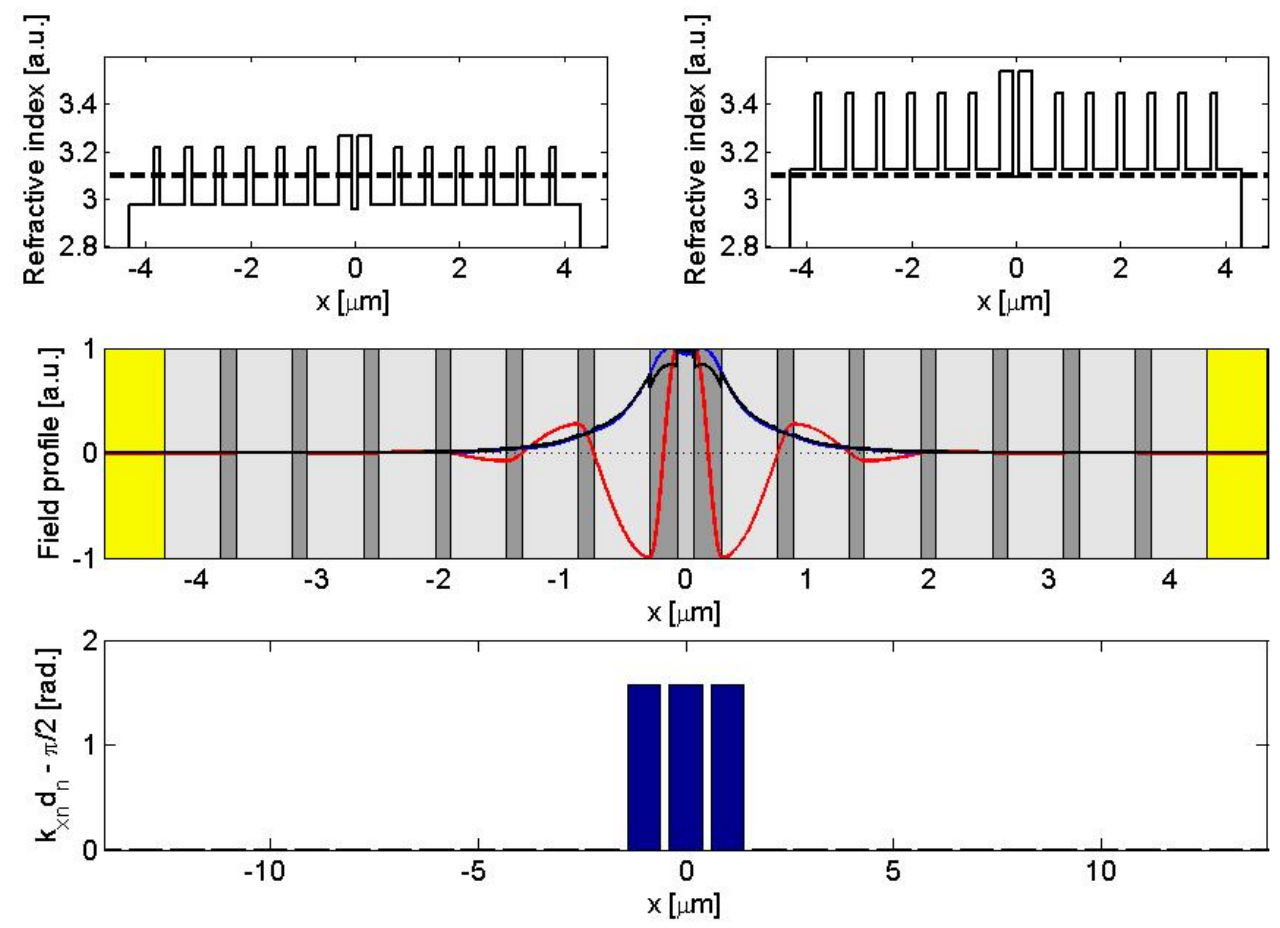

Figure 4.2 - Refractive index profiles of TIR (top left) and CIM (top right); mode profiles of TIR TE (blue), TIR TM (black) and CIM TE (red) for type II PM superimposed on the structure (middle); phases of the waves at the PM point in each layer (bottom).

of the wave at the phase matched point in each of the layers. Since the structure was designed with the quarterwave condition, the term " $\phi_{n}-\pi /$ " is zero for all the cladding layers. $\phi_{n}=k_{x, n} d_{n}$ where $\mathrm{n}$ is the layer for which the phase is to be calculated.

Table 4.1 lists all the parameters of this design.

\subsection{Structure Design of Coupled Interface Modes for Type I phase matching}

The design of section 4.2 leads to a structure that shall be kept set throughout the simulations for type I phase matching. This time, the wavelength will be swept in search the crossing between the two effective indices. After several trials, a point of intersection 
Table 4.1 - The Coupled Interface Mode design parameters for type II phase matching

\begin{tabular}{|l|l|}
\hline Parameter & Value \\
\hline$\left(x_{\text {core }}, x_{1}, x_{2}, x_{\text {cap }}\right)$ & $(0.85,0.3,0.8,0.2)$ \\
\hline$\left(n_{\text {core }}, n_{1}, n_{2}, n_{\text {cap }}\right)$ at $\omega$ & $(2.9549 \mathrm{e}, 3.2145,2.9777,3.2656)$ \\
\hline$\left(n_{\text {core }}, n_{1}, n_{2}, n_{\text {cap }}\right)$ at $2 \omega$ & $(3.0942,3.4444,3.1232,3.5381)$ \\
\hline$\left(t_{\text {core }}, t_{1}, t_{2}, t_{\text {cap }}\right)$ & $(140 \mathrm{~nm}, 127.44 \mathrm{~nm}, 463.29,224.87)$ \\
\hline$n_{\text {eff }}$ & 3.0954 \\
\hline Number of periods in top stack & 6 \\
\hline Number of periods in bottom stack & 7 \\
\hline
\end{tabular}

was found at $\lambda=1523.5 \mathrm{~nm}$ corresponding to a second harmonic at $\lambda=761.75 \mathrm{~nm}$. Fig. 4.3 shows the intersection between the refractive index of the TM second harmonic wave and the TE fundamental wave.

Refractive index profiles of TIR (top left) and CIM (top right); mode profiles of TIR TE ( blue), TIR TM (black) and CIM TE (red) for type II PM superimposed on the structure (middle); phases of the waves at the PM point in each layer (bottom)

Similar to the type II case, Fig. 4.4 shows the refractive index profiles of the structure at the two fundamental and second harmonic wavelengths. The effective index common to the two profiles is shown with a dashed line. This is the same point of intersection in Fig. 4.3 .

The mode profiles of all of TE fundamental and TM second harmonic are shown in Fig. 4.4, superimposed on the structure schematic. From the the phase plot at the bottom of Fig. 4.4, it is evident that since the design parameters were preset, the structure is no longer quarterwave and hence the phases of the waves inside the layers are not $\pi / 2$ anymore.

Table 4.2 lists all the parameters of this design for type I phase matching. 


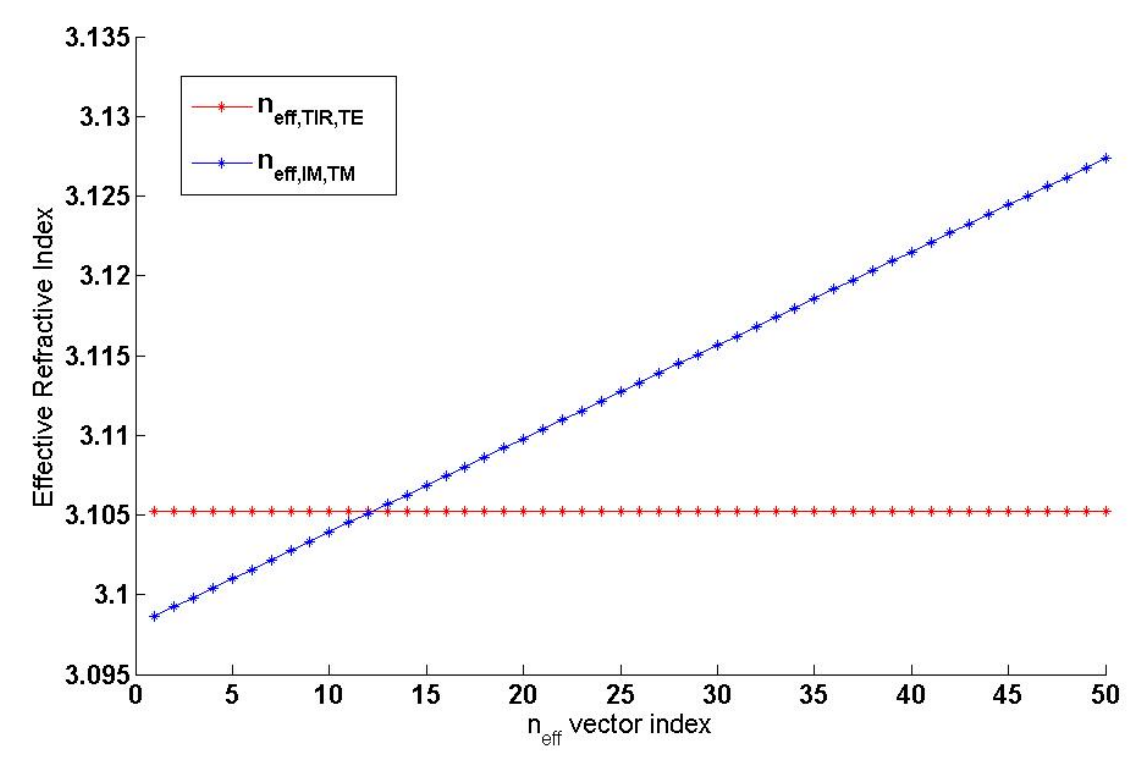

Figure 4.3 - The intersection of the TE fundamental wave refractive index and TM second harmonic indices for type I phase matching.
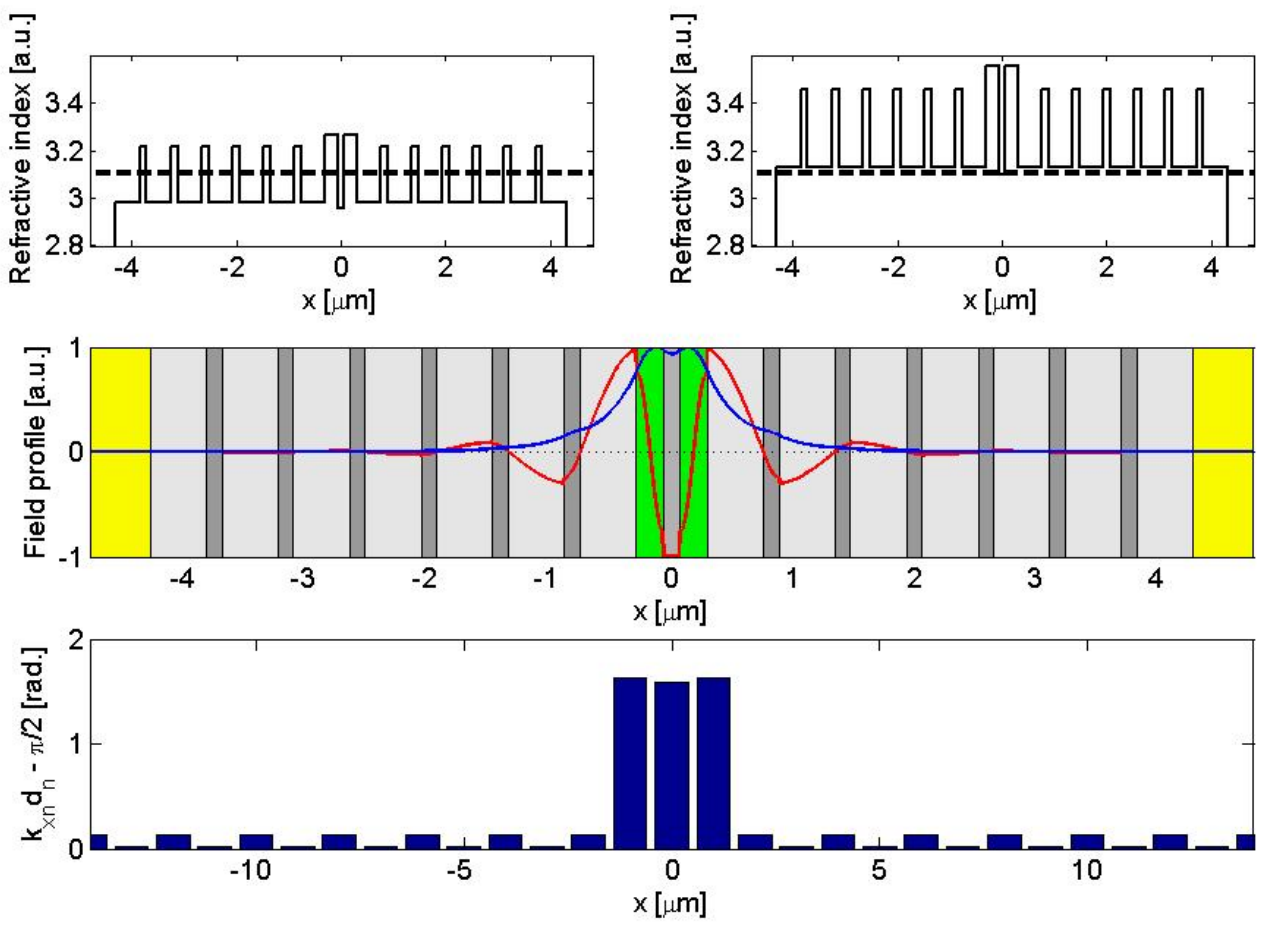

Figure 4.4 - Refractive index profiles of TIR (top left) and CIM (top right); mode profiles of TIR TE (blue)and CIM TM (red) for type I PM superimposed on the structure (middle); phases of the waves at the PM point in each layer (bottom). 
Table 4.2 - The Coupled Interface Mode design parameters for type I phase matching

\begin{tabular}{|l|l|}
\hline Parameter & Value \\
\hline$\left(x_{\text {core }}, x_{1}, x_{2}, x_{\text {cap }}\right)$ & $(0.85,0.3,0.8,0.2)$ \\
\hline$\left(n_{\text {core }}, n_{1}, n_{2}, n_{\text {cap }}\right)$ at $\omega$ & $(2.9558 \mathrm{e}, 3.2159,2.9787,3.2672)$ \\
\hline$\left(n_{\text {core }}, n_{1}, n_{2}, n_{\text {cap }}\right)$ at $2 \omega$ & $(3.0986,3.4531,3.1279,3.5522)$ \\
\hline$\left(t_{\text {core }}, t_{1}, t_{2}, t_{\text {cap }}\right)$ & $(140 \mathrm{~nm}, 127.44 \mathrm{~nm}, 463.29,224.87)$ \\
\hline$n_{\text {eff }}$ & 3.1052 \\
\hline Number of periods in top stack & 6 \\
\hline Number of periods in bottom stack & 7 \\
\hline
\end{tabular}

\subsection{Two dimensional Simulations of the CIM design}

The simulations carried out above, rely based on the assumption that the problem is that of one dimension. This would be the case if the structures were tested as slab waveguides; however, in order to increase the mode confinement, and hence the process efficiency, the waveguides will be etched to a certain depth. The lower refractive index of air around the ridge of the waveguides will then induce better confinement of the mode within the center of the waveguide.

The details of the fabrication procedure to etch the waveguides will be discussed in the next chapter. In this section, the already designed waveguide of the previous sections is considered in a $2 \mathrm{D}$ environment where the ridge waveguides can be etched with various ridge widths. Fig. 4.5 shows the mode profiles of both fundamental TIR and second harmonic CIM superimposed on the $2 \mathrm{D}$ structure. The etch depth of the design is set to assure confinement of the mode while not passing further than an extermum in the mode profile. In the case of the designed coupled interface modes, the lobes in the mode profile occured at the boundary between the cap layer and the first layer of the claddings; as a result, the waveguides were only etched upto the top cap layer as shown in Fig. 4.5. Also, 

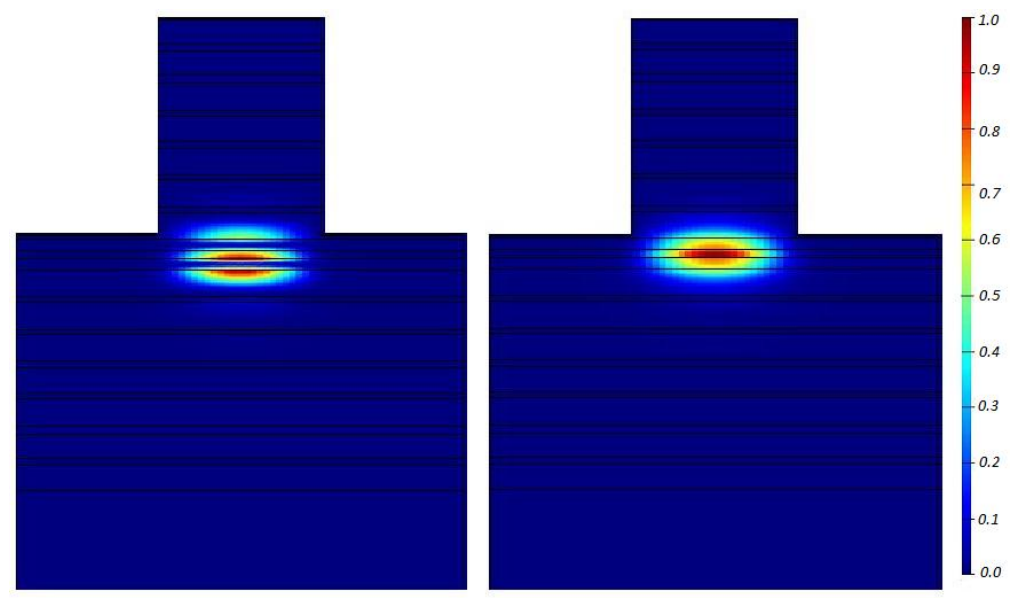

Figure 4.5 - Two dimensional mode profile of fundamental (right) and second harmonic (left) waves coexisting in the periodic layered medium.

since the interface mode is more lossy and less confined than the TIR mode, etching only the top stack affects the symmetry of this mode, more prominantly than it does the TIR mode.

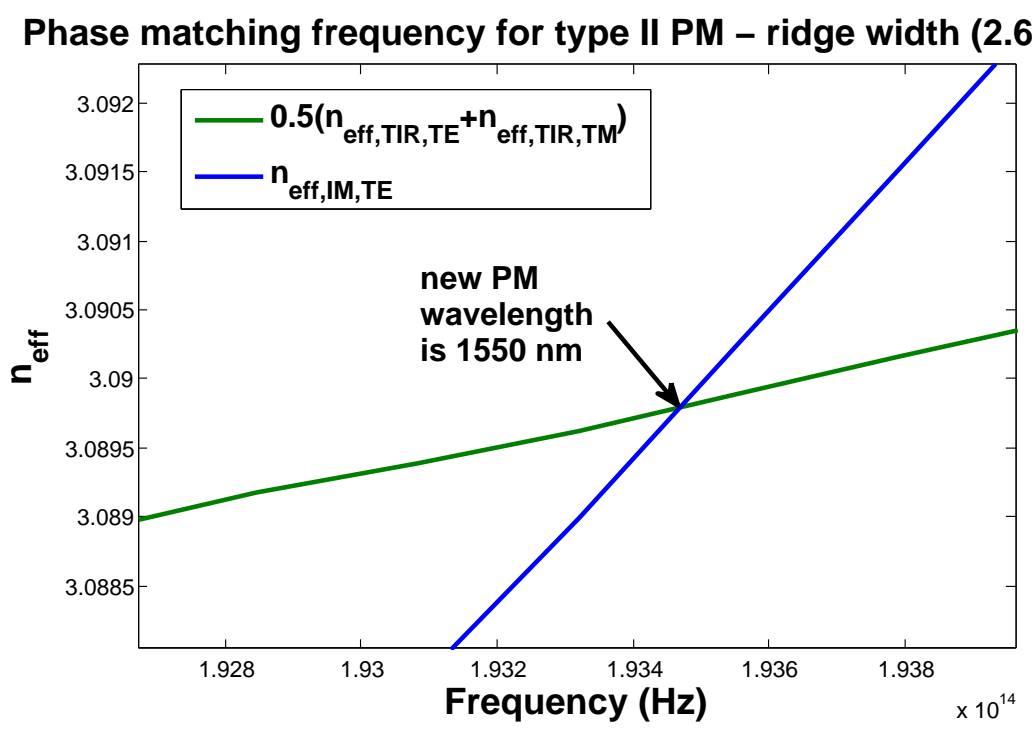

Figure 4.6 - Type II phase matching wavelength shift for a $2 D$ waveguide with a ridge width of 3 .

The main purpose of these 2D simulations are to determine the new, shifted phase matching wavelength for each PM type. The reason for this shift is the fact that the 
refractive indices that the mode "sees" are now also shifted as a result of the surrounding air around the ridge. The dispersion diagrams of $n_{\text {eff }}$ versus frequency were generated using Lumerical Mode Solutions [41]. Fig. 4.6 to 4.10 show the shift in the wavelength for ridge widths of $2-5 \mu m$.

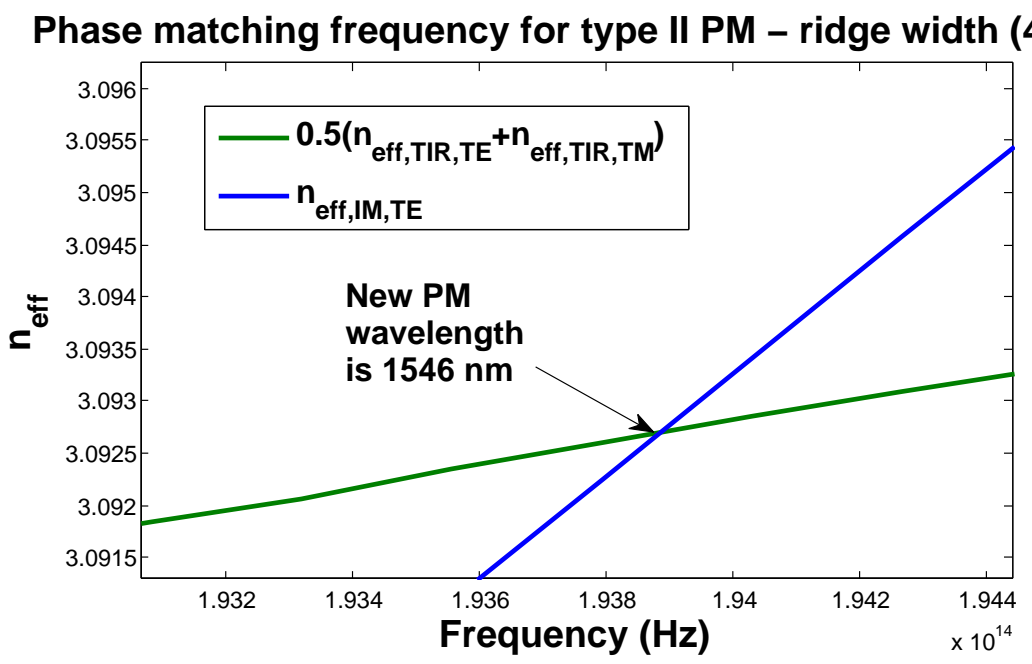

Figure 4.7 - Type II phase matching wavelength shift for a $2 D$ waveguide with a ridge width of $4 \mu \mathrm{m}$.

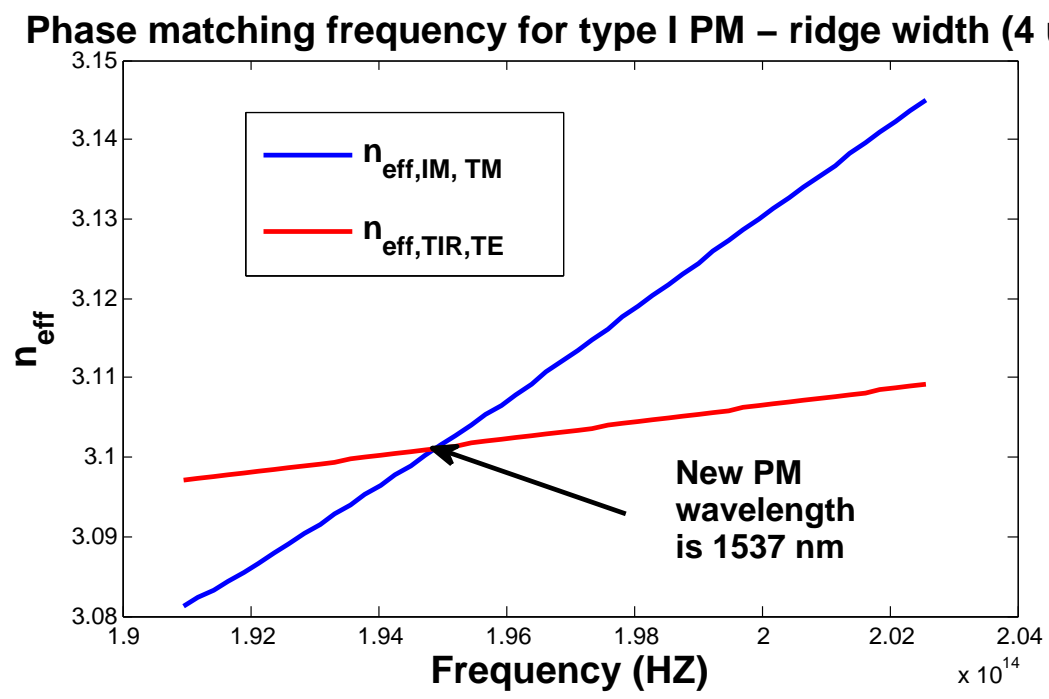

Figure 4.8 - Type I phase matching wavelength shift for a $2 D$ waveguide with a ridge width of $3 \mu \mathrm{m}$. 


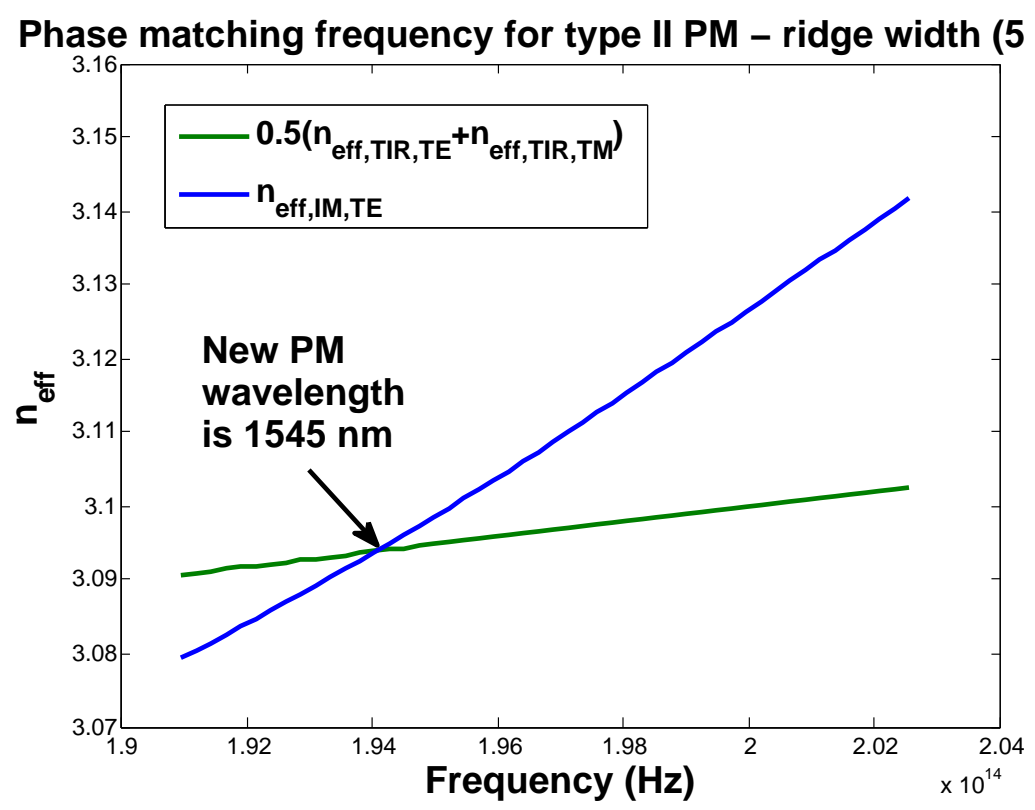

Figure 4.9 - Type II phase matching wavelength shift for a $2 D$ waveguide with a ridge width of $5 \mu \mathrm{m}$.

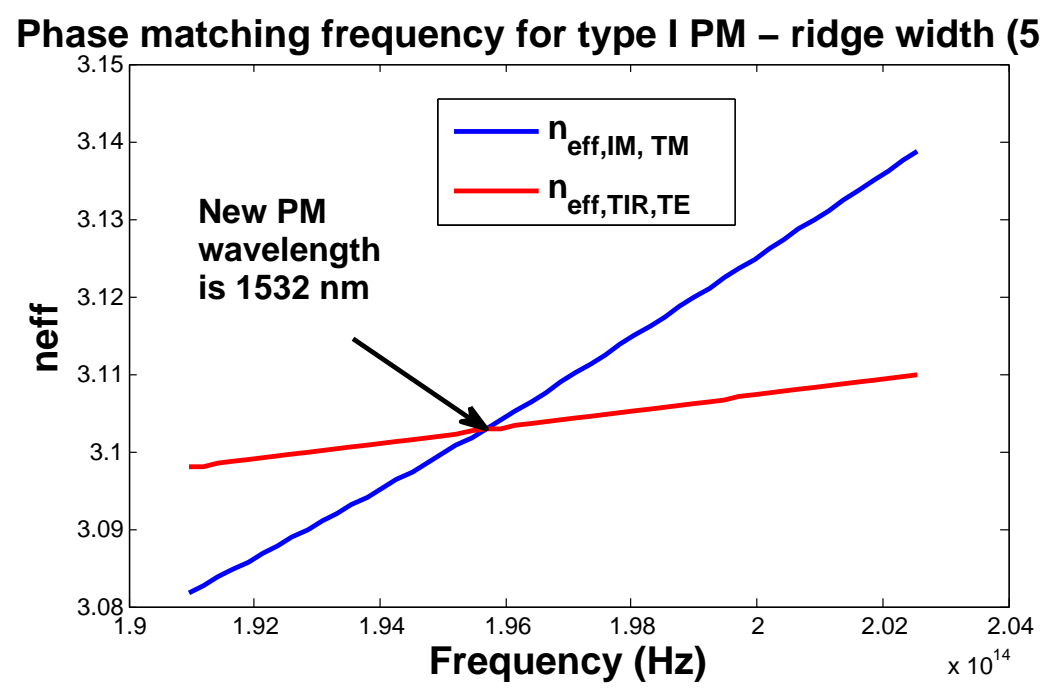

Figure 4.10 - Type I phase matching wavelength shift for a $2 D$ waveguide with a ridge width of $5 \mu \mathrm{m}$.

From the figures, it is evident that the smaller the width of the ridge waveguide becomes, the further the phase matching wavelength will be shifted towards longer wavelengths. These new PM points will be used to estimate the second harmonic wavelength 
of the practical devices for the purpose of characterization. In the later chapters, the fabrication process of this design will be reviewed and the characterization results of the devices and their agreement with the theoretical predictions will be discussed. 


\section{Chapter 5}

\section{Device Fabrication}

The fabrication process of the nonlinear waveguides desgined and optimized in the previous chapters involves several steps: growing the layers of the structure via chemical vapour deposition and then patterning and etching the wafers to obtain ridge waveguides providing better confinement. The addition of the ridges turns the analysis from a one dimensional problem to one in two dimensions. As a result, the effective refractive indices, wave numbers and hence the phase matching wavelength shift from their original values to new ones as predicted by the $2 \mathrm{D}$ simulations in Chapter 3.

This chapter discusses wafer specifications and reviews the fabrication procedure involved in making the ridge waveguides that accommodate interface modes.

\subsection{Wafer Growth}

The wafer was grown, nominally undoped, using metal-organic chemical vapour deposition on a GaAs substrate. A layer of $500 \mathrm{~nm} \mathrm{Al}_{0.85} \mathrm{Ga}_{0.15} \mathrm{As}$ was grown as a buffer on top of the substrate. The structure was composed of a $140 \mathrm{~nm}$ thick core layer of $\mathrm{Al}_{0.85} \mathrm{Ga}_{0.15}$ As followed on each side by a $224.9 \mathrm{~nm}$ thick $\mathrm{Al}_{0.20} \mathrm{Ga}_{0.80} \mathrm{As}$ cap layer. The top and bottom claddings consisted of 6 and 7 periods respectively, of alternating high and low aluminium concentration AlGaAs. Each period contained a layer of $127.4 \mathrm{~nm}$ 


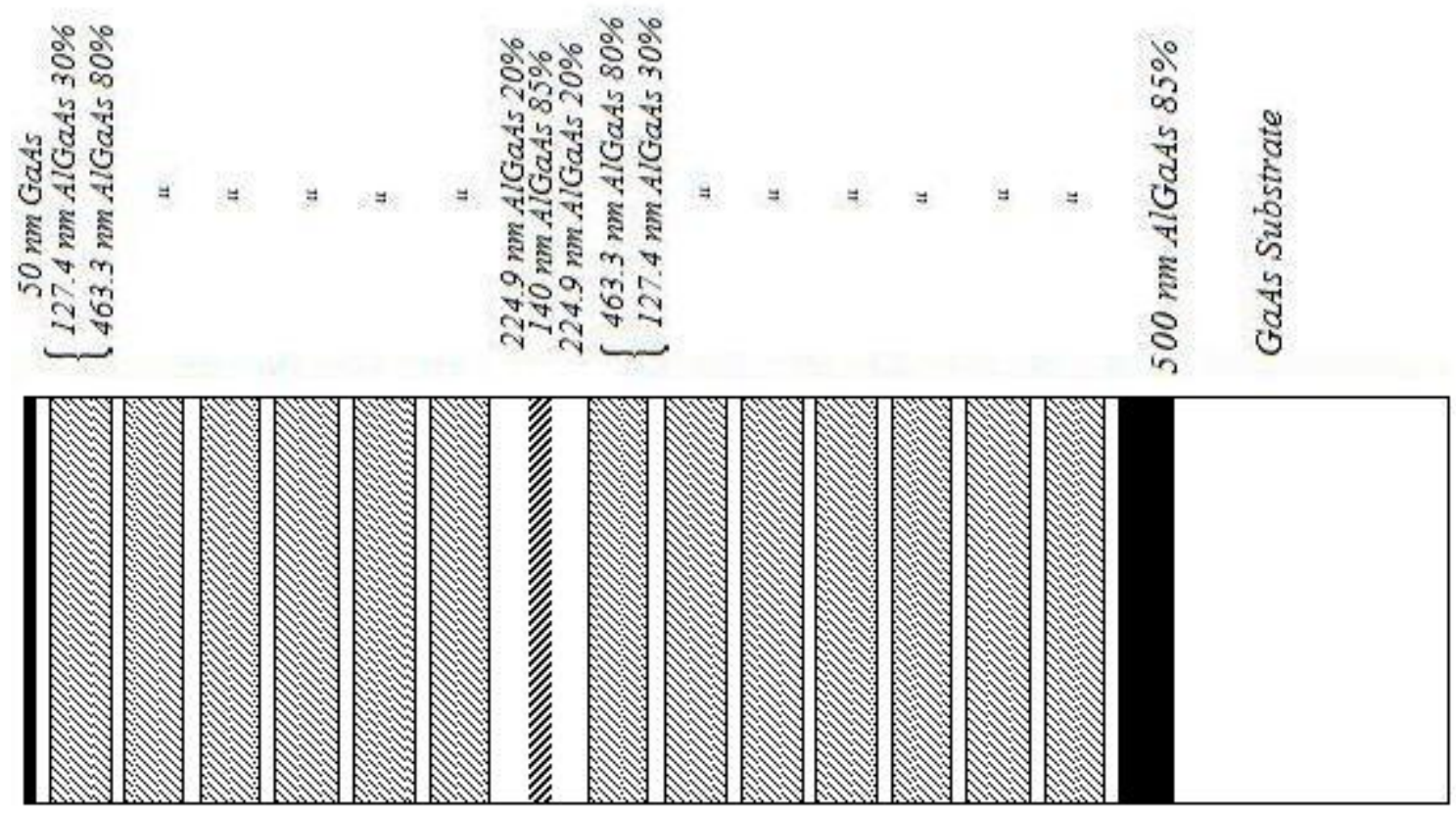

Figure 5.1 - A schematic of the cross section of the structure; the bottom stack has one more period than the top stack.

thick $\mathrm{Al}_{0.30} \mathrm{Gaa}_{0.70} \mathrm{As}$ and a layer of $463.3 \mathrm{~nm}$ thick $\mathrm{Al}_{0.80} \mathrm{Ga}_{0.20}$ As. Fig. 5.1 depicts a schematic of the cross section of the structure.

\subsection{Waveguide Fabrication}

Fabrication of the waveguides involves several steps of careful micro-manufacturing. This section reviews the fabrication procedure of a ridge waveguide starting from a wafer; first, an analysis of the etch mask production, then the etching and thinning processes and finally some associated fabrication issues.

\subsubsection{Etch Mask Preparation}

In order to etch a wafer, there needs to be an etch mask that transfers a certain pattern onto the wafer; a mask that resists the etchant particles, hence protecting the maskcovered portions of the wafer. This mask needs to be initially deposited and patterned 
on top of the wafer. Photoresist $(\mathrm{PR})$ is a photosensitive chemical used as this mask. Patterning this mask requires optical lithography, a step that exposes the photoresist to a UV radiation through a patterned mask, changing its chemical properties such that a developer can recover the patterns transferred onto the photoresist layer. Once the patterns are generated, the photoresist can serve as a mask and withstand the etch.

For better process robustness and in order for the mask to withstand etchant gases more efficiently, a second support mask in the form of an oxide layer is added between the photoresist and the wafer. Consequently, the photoresist is used to pattern the oxide layer which is in turn used to pattern the wafer. The oxide layer is known to better resist the removing power of the etchant gases. Furthermore, the photoresist is not removed until after the etch step is complete, effectively increasing the mask height as well.

The proceeding sections discuss and explain the physics behind each of the steps required to fabricate the etch mask.

\subsubsection{Oxide layer deposition}

The Silica deposition step is the first step in mask preparation as mentioned above. In order to achieve a uniformly deposited layer of $\mathrm{SiO}_{2}$ as thin as a few hundred nanometers, Plasma Enhanced Chemical Vapour Deposition (PECVD) is used. $\mathrm{SiH}_{4}$ and $\mathrm{N}_{2} \mathrm{O}$ gases enter the PECVD chamber where they get excited and ionized by an existing plasma, producing reactive silicon and oxygen. At the high operating temperature of the PECVD, these gases react to produce $\mathrm{SiO}_{2}$.

For this experiment, $200 \mathrm{~nm}$ of Silica was deposited at $400 C$ on top of the wafers. This oxide layer is next patterned using photoresist and optical lithography.

\subsubsection{Photoresist spin coating and Optical Lithography}

The fabrication recipes used throughout this project have been developed by B. J. Bijlani. Slight modifications have been made to the recipes to accomodate the particular needs of 
the waveguides studied here. Furthermore, the lithography process including photoresist deposition, exposure and development is an optimized variation of results of work done prior to this project for the author's undergraduate thesis [42] in conjuction with Bijlani's developed recipes. The lithography optimization from [42] involved experimenting with several photoresist materials and different spinning, exposure and developing variables to produce consistent rectangular PR that would resist the etchant gases best. Fig 5.2 shows some of the results of this work. The Scanning Electron Micrographs (SEMs) show cross sections of developed photoresist with different development times. It was found that as the development time increased, a V-shaped cut on top of the photoresist started to form and the perfect rectangular shape of the photoresist cross section started to fade away, deteriorating its quality for etch mask utilization.

The photoresist used throughout the experiments is S1818, positive, by Shipley Inc. [43], the best performing of the several investigated in [42]. A positive photoresist is one that is affected by the incident UV light and chemically removed during development. Conversely, a negative photoresist is one that becomes resistant against the developer upon exposure to UV light and is developed otherwise. The application of the photoresist onto the wafer surface is done through spin-coating. A thin layer of a primer material is spun onto the sample at a fixed speed and acceleration rate. The primer is a coating spun prior to main photoresist coat, to improve the bonding between the substrate, in this case the oxide layer, and the subsequent coatings, in this case the photoresist. Later, PR is dispensed onto the wafer surface in drops. The spinner uses a centrifuge force and spins at a preset speed rate for a preset amount of time. This spin speed rate and time affect the thickness of the coated photoresist and were already optimized for the purposes of this work: maximized thickness while maintaining effective transfer of the pattern onto the photoresist and achieving vertical side walls. Here, S1818 was spun on the wafers at a speed of $4000 \mathrm{rpm}$ for 45 seconds.

The PR coated samples are next exposed to UV light through Optical Lithography. 

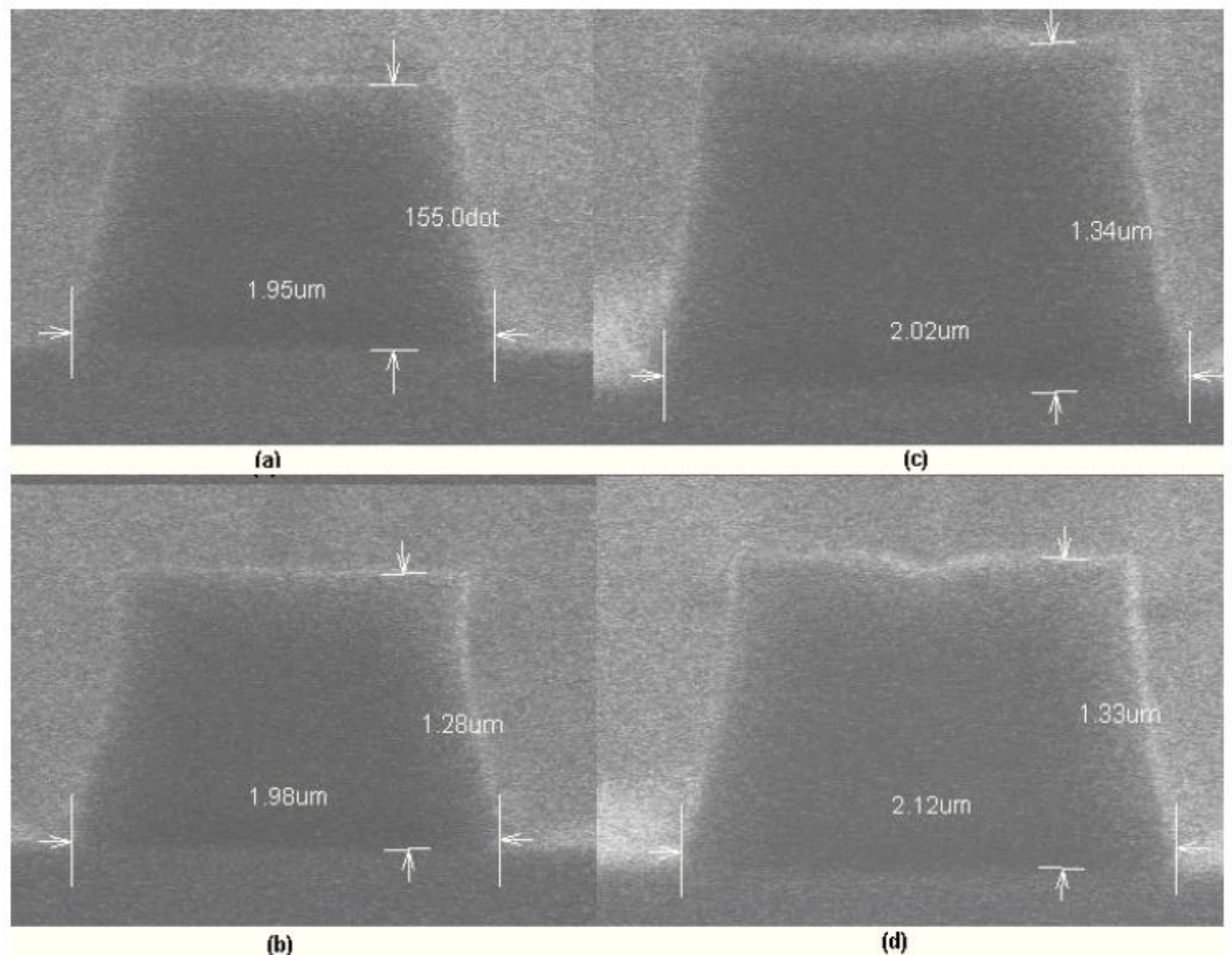

(b)

(d)

Figure 5.2 - SEMs of photoresist ridges as a function of development times (a)50 s,(b)60 $s,(c) 70 s,(d) 80 s$.

Optical lithography is the series of steps performed on the PR in a mask aligner/exposure tool to pattern the photoresist. A pre-manufactured glass mask is placed in between the radiation source and the wafer. The desired patterns are pre-written on the mask. These patterns will be transferred onto the photoresist through UV exposure. Optical systems on the rays' paths are used to focus and/or help resize the pattern since some processes require larger or smaller pattern sizes than is available on the masks. In this work, no resizing was performed.

As the UV light is shone onto the wafer, it passes through the mask. The UV rays then are blocked by the dark pattern lines on the glass mask, but pass through everywhere else. If a spot on the photoresist is exposed to these rays, the cross links of the polymer are 

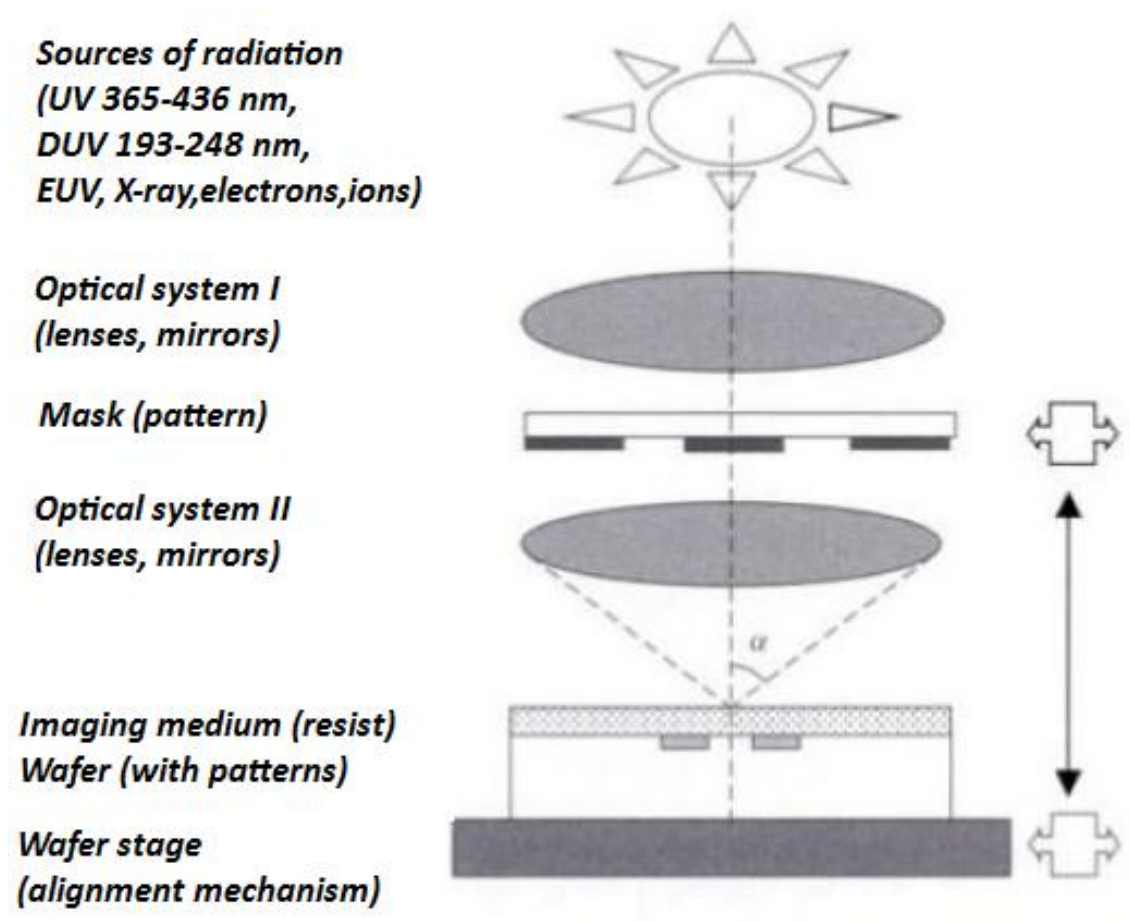

Figure 5.3 - Schematic of a Mask Aligner.

broken and a developer can solve that portion of the PR. Fig. 5.3 [51] shows a schematic of an optical lithography equipment also known as a mask aligner.

By setting the exposure gap and time on the aligner, one can vary the amount of chemical change that the photoresist undergoes. Consequently, one has control over the photoresist development quality and pattern transfer consistency. For the purposes of this work, the PR treated wafers were exposed to 6.5 seconds of UV light with a $50 \mu \mathrm{m}$ gap from the mask. The mask contained several periods of lines that varied in width. Each period consisted of 8 lines varying in width from $2 \mu m$ to $5 \mu \mathrm{m}$. Lines two through seven were best developed and the corresponding waveguides were used in characterization.

Once the exposure procedure is done, the wafers are ready to be developed. The amount of time a sample is in contact with the developer can influence the resulting developed pattern. Generally, the longer a sample is developed, the narrower the resulting pattern lines will be. The developer used in this project was MF-321 by Shipley Inc. [44], and the wafers were immersed in the developer for 60 seconds for optimum pattern 
transfer.

Upon developing the patterns, the wafer is hard baked. Hard baking is the process of post development heating of the wafers and UV-cross linking then to improve the etch resistance of the PR and minimize out-gassing [45]. The temperature and length of this heating step is critical in that overheating can melt the photoresist.

\subsubsection{Plasma Etching of the Oxide Layer}

The PR patterns are transferred onto the oxide via plasma etching. Etching is the process through which an "unprotected" substrate is exposed to active particles and reacts with them generating products that are removed from the etch chamber. Two methods are simultaneously employed to etch the oxide layer in this project: Reactive Ion Etching (RIE) and Inductively Coupled Plasma (ICP). In RIE, RF fields are applied through a powered electrode to the chamber exciting the reactive gases that enter the chamber. Consequently, ions and excited particles coexist in the chamber. The energy of these ions is about a few hundred electron volts [46]. An alternative to RIE is the use of ICP to achieve higher plasma densities. In the Inductively Coupled Plasma method, the RF power is applied through coils encircling the chamber and producing strong electric fields which in turn induce magnetic fields that retain the particles in the centre of the chamber to help produce high ion densities [46].

These two different power mechanisms move the ions and particles in certain directions in the chamber to enhance the chemical and physical reactions with the surface of the substrate. As the power is increased, the energy with which the particles are excited is also increased and hence the etch rate is enhanced; however, an increased RIE power could cause physical bombardment of the surface that is hard enough to produce roughness. This side wall roughness could potentially increase the scattering losses [47]. High ICP powers on the other hand, will give rise to undercuts in the etch. A sample undercut is illustrated in Fig. 5.4 [48]. Undercuts are step shaped edges that form when a sample is 
over etched. They result from the irresistibility of the PR and its failure towards very high power at long etches.

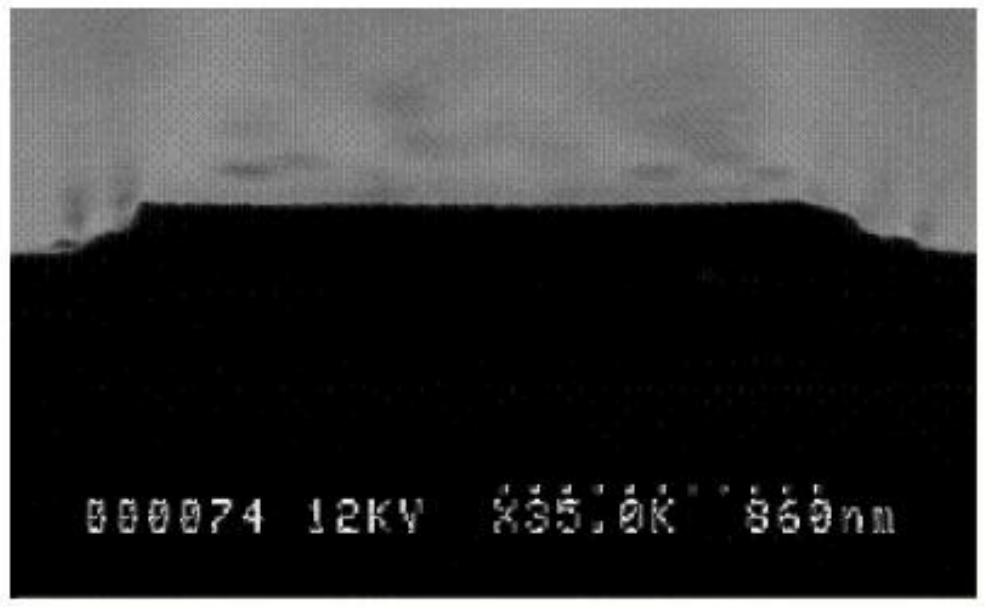

Figure 5.4 - SEM of an etch undercut on SiO2 formed due to an ICP overetch.

At relatively low pressures there are fewer particles present inside the chamber. As a result the mean free path of an ion will increase. The mean free path is defined as the length travelled by an ion before being scattered by a particle [49]. If the probability of particle collisions decreases, the time for the ion to accelerate and hit the surface of the sample without being scarred away is shortened.

$\mathrm{CHF}_{3}$ is the key etchant gas present in the chamber for etching $\mathrm{SiO}_{2}$. Reactions induced by ion bombardment produce $\mathrm{CO}_{2}$ by-products that are removed from the chamber along with $\mathrm{SiF}_{4}$. Helium is also used to physically bombard the surface of the wafer. Fig. 5.5 shows the series of steps involved in patterning the oxide layer starting from the oxide deposition.

Each gas was pre-assigned a flow rate at which it enters and exits the chamber. Generally, there has to be a balance between pressure and flow rate; but occasionally, non-reactive gases like $\mathrm{N}_{2}$ are used to increase the pressure without modifying the gas flow rates. Higher flow rates in general help remove the volatile products from the bottom of the chamber. This improves the etch quality and increase the etch rate. 


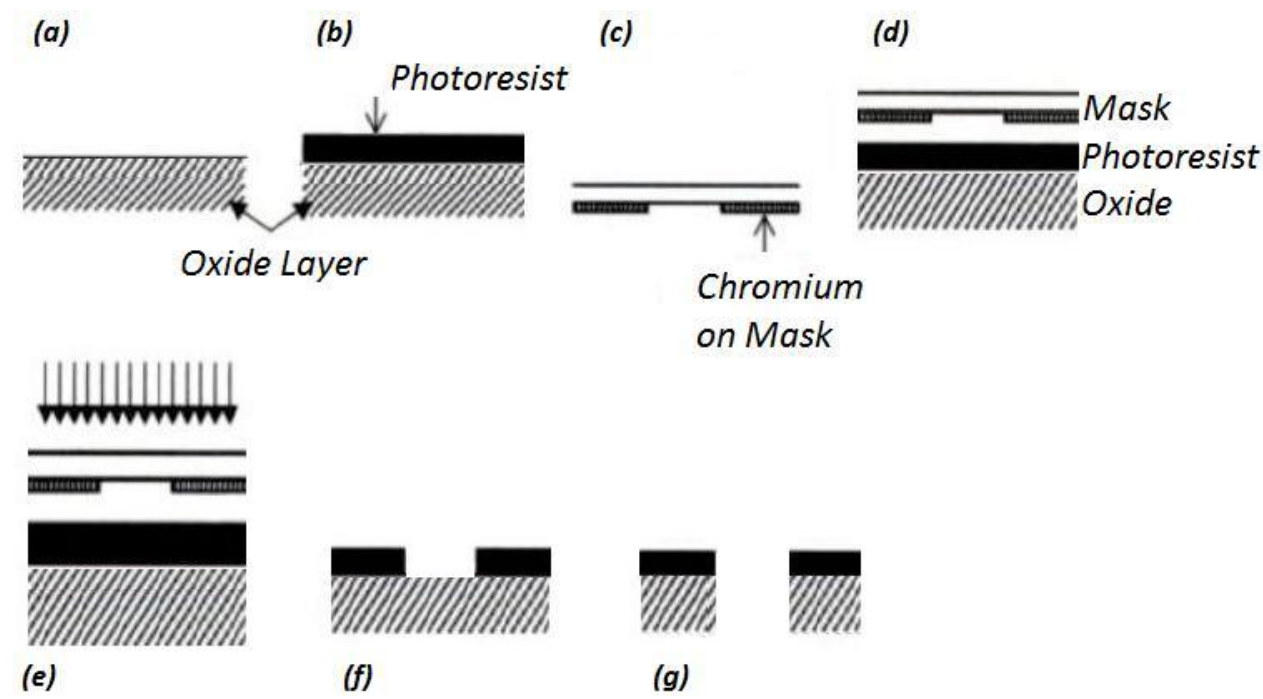

Figure 5.5 - Photolithography :(a) The oxide layer on top of the substrate (b) A layer of photoresist spun on top of the oxide layer (c) The Chromium patterns on the glass mask (d) The alignment of the mask and the wafer (e) UV radiation through the mask onto the resist layer (f) The developed positive resist layer with the transferred pattern ( $g$ ) The non-covered portion of the oxide, plasma etched.

In this experiment, $\mathrm{SiO}_{2}$ was etched by $\mathrm{CHF}_{3}$ at a flow rate of $50 \mathrm{sccm}$, and $\mathrm{He}$ at a flow rate of $8 \mathrm{sccm}$ with an RIE power of $70 \mathrm{~W}$ and an ICP power of $400 \mathrm{~W}$ for 80 seconds.

The wafers were then immersed in a 10 to 1 mixture of ammonium fluoride and $\mathrm{HF}$ acid, for a buffered oxide etch (BOE). This wet etching follow up ensures a clean and residue-free AlGaAs surface, ready to be etched with silica as the mask.

\subsubsection{Plasma Etching of the wafers}

As discussed earlier, PR and the oxide layer both act as an etch mask, transferring the pattern on to the wafer. This is mainly due to the failure of photoresist to mask the substrate for the entire duration of the etch for etch depths longer than a micron. Fig. 5.6 shows a scanning electron micrograph of GaAs etched for less than a micron using only photoresist as the mask. As seen in the image, the resist is starting to get etched 


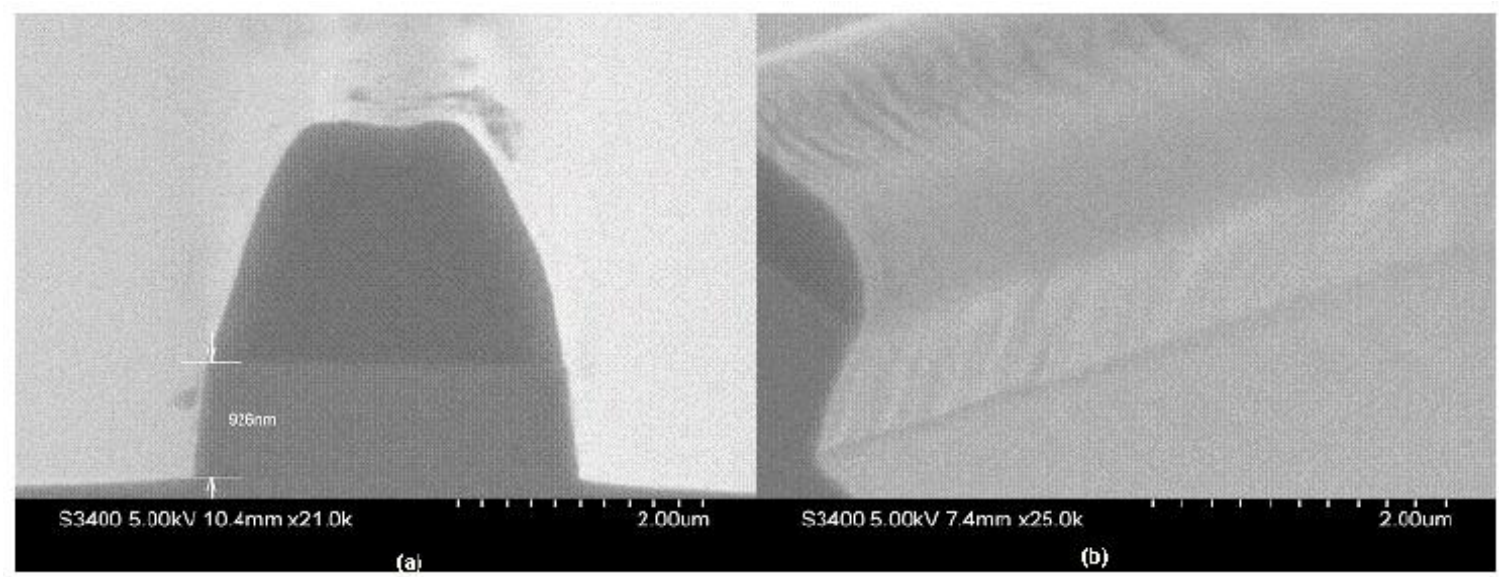

Figure 5.6 - SEM of etched GaAs using only photoresist.

away and would not withstand a deeper etch. Chemical and physical reactions have to take place to etch the wafer and hence resolve the pattern. Depending on the substrate chemical and physical make up, different etch methods are employed. These methods are most commonly divided into the three groups of wet, dry and plasma etching; however plasma etching is occasionally categorized under dry etching.

In this project, the $\mathrm{AlGaAs}$ etching was done using plasma etching with $\mathrm{BCl}_{3}$ and Ar particles. A combination of RIE and ICP plasma etch methods is used to etch the wafer. The rest of this section reviews the plasma processes and parameters involved in the AlGaAs etch.

Equations 5.1 to 5.3 show examples of the formation of ionized Argon species, excitation of $\mathrm{BCl}_{3}$ Molecules and their reaction with AlGaAs [50].

$$
\begin{gathered}
e^{-}+A r \rightarrow A r^{+}+2 e^{-} \\
2 B C l_{3} \rightarrow B_{2} C l_{4}+2 C l, 2 B_{2} C l_{4} \rightarrow B_{4} C l_{4}+2 C l \\
A l_{O .5} G a_{O .5} A s+3 C l \rightarrow 0.25 \mathrm{Al}_{2} C l_{6}+0.25 G a_{2} C l_{6}+0.25 A s_{4}
\end{gathered}
$$

This project involves searching for a highly selective, anisotropic, smooth and uniform AlGaAs etch. The etch rate was not of concern for this project since the maximum etch 
time for the experiments was about 25 minutes.

The reactions provide no directionality, meaning the etch rates in both the vertical and horizontal directions are equal. This is referred to as isotropy. Isotropy is not desired for the waveguide etches here. Ionized particles are responsible for the physical bombardment of the substrate surface. These particles are accelerated by the RF field [51]. This physical bombardment causes directionality since the field is only applied in the vertical direction. The anisotropic etch resulting from these reactions produces vertical sidewalls.

Selectivity of one material over the other in an etch process evaluates how well the PR can resist the etchant gases. The chemical reactions are usually highly selective. It is only the physical ion bombardment that might damage the resist.

An etch process can take anywhere from less than a minute to hours depending on the gases, the substrate, and the application. Generally, the higher the etch rate, the more preferred the etch recipe.

Uniformity denotes two things: the consistency of the etch throughout the substrate surface and the extent to which the etch rate and quality is maintained from one wafer to the other [52]. Possible non-uniformity causes are chamber geometry, non-uniform gas composition and non- uniform applied field.

The optimized etch settings were, $8 \mathrm{sccm}$ Ar flowrate, $4.5 \mathrm{sccm} \mathrm{Cl}_{2}$ flowrate and 5 sccm $\mathrm{BCl}_{3}$ flowrate with $200 \mathrm{~W}$ ICP power, $50 \mathrm{~W}$ RIE power at a chamber pressure kept at 5 mTorr.

The etch recipe was run in a pulsed- operation mode, meaning that the plasma was on for 90 seconds and then off for 180 seconds to let the samples cool down in between successive etch periods.

Fig. 5.7 shows a SEM of the structure etched to a depth that corresponds to the point just before the high intensity mode lobe, to form a ridge waveguide. The ridge widths were varied from $2.65 \mu \mathrm{m}$ to $4.65 \mu \mathrm{m}$. 
Figure 5.7 - A SEM of the wafer structure. The layer marked as "center" is the core of the waveguide and the wafer is etched upto a mode profile extremum.

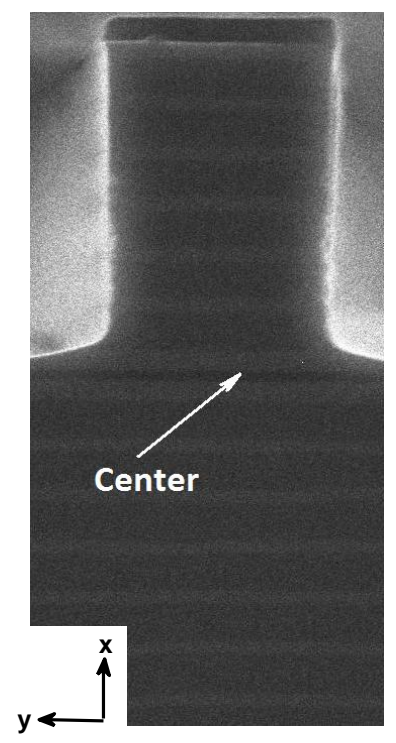

\subsubsection{Wafer Thinning and Cleaving}

Once the etch process is performed, masks can be removed and the wafers were ready to be thinned. The thinning is a necessary step in preparing the wafers for accurate cleaving.

Chemical-Mechanical Polishing (CMP) is used to thin the wafers. The CMP equipment consists of a rough polishing pad glued on top of a flat platen, a wafer chuck that holds the wafer upside down and polishing slurry.

As the platen rotates, the slurry is slowly introduced onto the pad. The chuck keeps the wafer at a pressure and the slurry spreads between the surface of the pad and the wafer. Slurries contain abrasive particles of varying sizes and adjusted-pH fluids that polish the wafer surface physically and chemically respectively. The removed thickness of the wafer depends on the slurry make-up and the thickness of the backing film placed underneath the wafer to adjust the extend to which the wafer will be thicker than the chuck surface. Fig. 5.8 shows a schematic of the equipment [53]. 


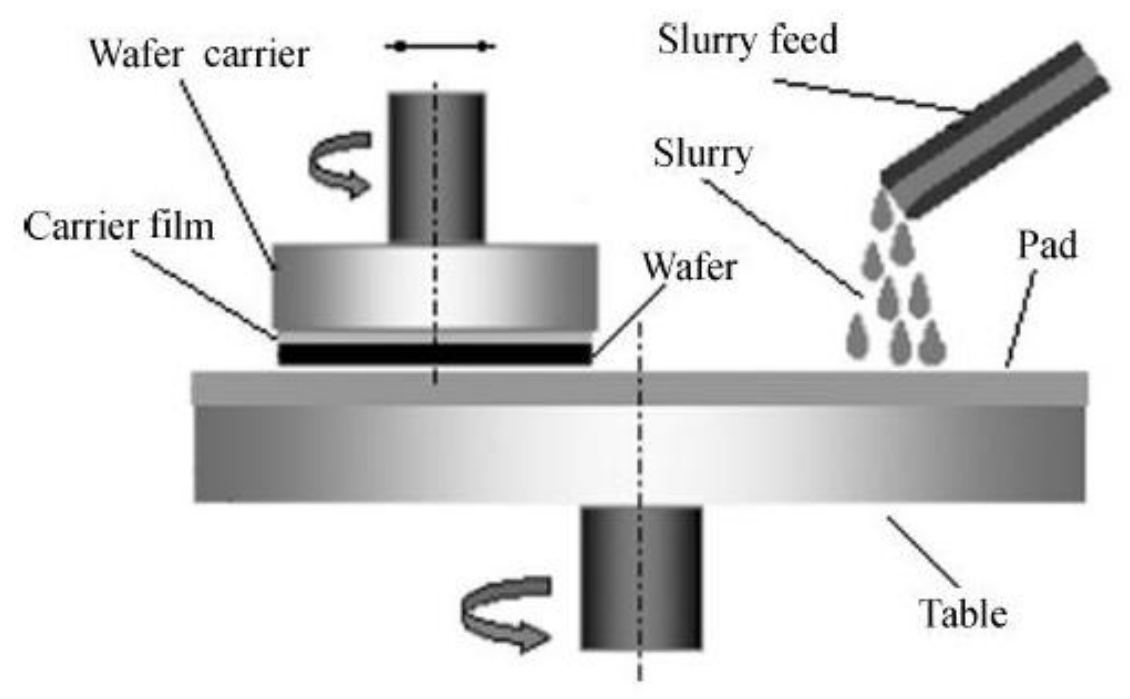

Figure 5.8 - Schematic of Chemical Mechanical Polishing.

\subsection{Fabrication Obstacles}

The fabrication process as described above occurs in a rather idealized situation. In practice, every single step is prone to real world non-idealities and limitations. This section discusses some of these issues and suggests solutions to overcome them.

The lithography procedure consists of the spinning, exposure and development steps. Throughout the spinning process, parameters such as spinning chamber humidity, chemical particles in the air and the wafer/photoresist age influence the quality of the coated layer. These factors are not controlled for precise spin conditions each time. Consequently, in order to minimize these effects every group of samples was fabricated within 24 hours. For example, the samples used for characterization of the waveguides with respect to a parameter such as ridge width, were all fabricated on the same day, or as close in time as possible.

Whilst these and other irregularities could take place during the spinning and the development to cause inconsistency in the results, the exposure step errors are the most dominant. The mask aligner used in this project enables the user to align the sample to the mask patterns under a microscope with a $X 50$ objective. While this technology 
is an advantage to this project in comparison to other conventional aligners, it still does not provide $100 \%$ accuracy in measurements. For the step of etch mask fabrication, this problem limits the resolution available to adjust the pattern lines perpendicularly to the sample edges. This practice is necessary for achieving a straight waveguide which in turn is associated with maximum coupling efficiency; as such, assuring consistency between multiple fabrication sets becomes rather difficult.

Developing the exposed photoresist also involves errors that influence the robustness of the fabrication process. Eventhough the recipe specifies a certain amount of development time, a common practice is to let the samples sit in the developer for as long as one decides is appropriate by observing the patterns as they develop. This renders the process relative and causes variations from one sample to another.

Finally, the etching step for both the oxide and the wafer are also subject to the same uncontrollable parameters as those in the spinning step. The chamber/air humidity and existing particles greatly affect the etch results. Solutions for these variations include carrying out full experiments in one day as well as running optimized "clean" recipes on the etcher before the actual etch recipes to provide a consistently clean chamber everytime.
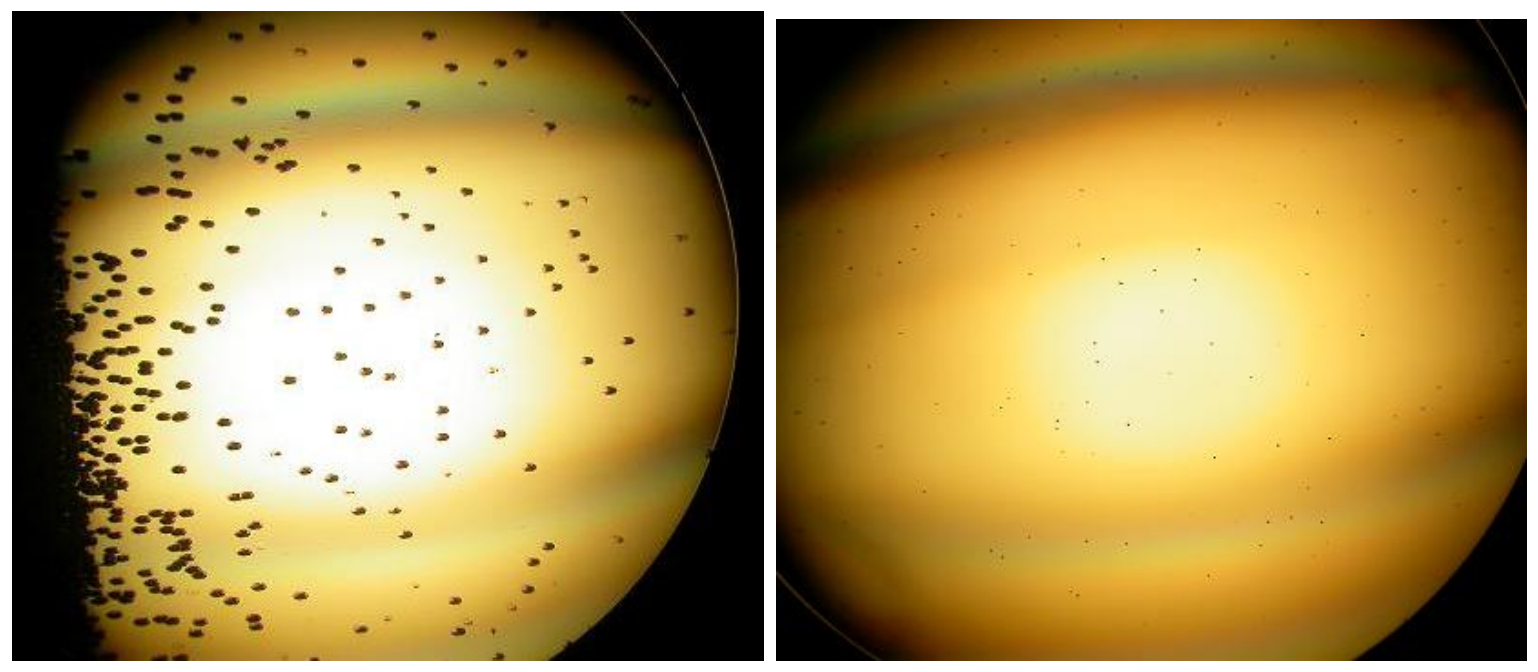

Figure 5.9 - Optical micrographs of wafer edge (left) and center (right) surface defect. 
Apart from the waveguide fabrication obstacles, the wafer in this case also suffered from growth irregularities. Fig. 5.9 shows an image taken by an optical microscope of the surface of the wafer. The great defect density seen in this image is explained by the stress put on the layers as a result of stacking layers of AlGaAs with aluminium concentrations of great contrast on top of one another. In particular, it is believed that the last $30 \%$ AlGaAs layer in the bottom stack on top of the $85 \%$ AlGaAs buffer layer causes a lot of stress in the wafer surface due to lattice mismatch. The high defect density around the edges of the wafer made it impossible to fabricate non-defective waveguides from those areas. As a result, the samples were carefully chosen from the center of teh wafers such that the average defect density of each sample was minimum and consistent with other samples.

Despite all the challenges concerning the fabrication of the waveguides, a relatively robust process was developed and applied to produce thinned ridge waveguides. Following the thinning process, the AlGaAs wafers were ready to be cleaved into pieces of 1-3 $\mathrm{mm}$ long waveguides. The thin wafers were easily cleaved under an optical microscope with the aid of a diamond scriber. The cleaved samples could then be characterized for device performance. 


\section{Chapter 6}

\section{Device Characterization}

The waveguides fabricated according to the steps in the previous chapter were later characterized for their linear and nonlinear performance. In this chapter, the results of this characterization are reported. These results are mainly used to prove the existance as well as the capability of CIMs to take advantage of second order nonlinearity of semiconductors.

\subsection{Loss Measurements}

Linear losses of the waveguides were measured using a Fabry-Prot method; a tunable distributed feedback laser source, "Velocity TLB-6300 tunable laser by New Focus" was tuned around the fundamental wavelength of $1550 \mathrm{~nm}$ to obtain device losses. Fig. 6.1 shows the schematic of the experimental set-up used for measuring this loss. The laser beam passes through a half wave plate (HWP) and a polarization beam splitter (PBS) adjusted to output the desired polarized light. The input pump power is then measured using a Germanium photo-detector (PD) "Newport 883-IR". Next, the beam passes through an input objective lens and is incident on the facet of the waveguide. The beam then exits the waveguide and is focused using an objective lens. A flip mirrors reflects the light towards a charge coupled device (CCD) camera, used to ensure maximum coupling 
out of the waveguide. If not reflected by the flip mirror, the beam is guided towards another Germanium photo-detector for measurement of the fundamental output power.

Fig. 6.2 shows a typical plot of the Fabry-Perot transmission spectrum of a $1.62 \mathrm{~mm}$ long waveguide. Using the length of the waveguide, the reflection from the facets and the maximum and minimum values of the transmission curve, fundamental wave loss can be calculated [54]. The lowest loss values were recorded for a ridge width of about $2.76 \mu \mathrm{m}$. Typical values were around $2.71 \mathrm{~cm}^{-1}$.

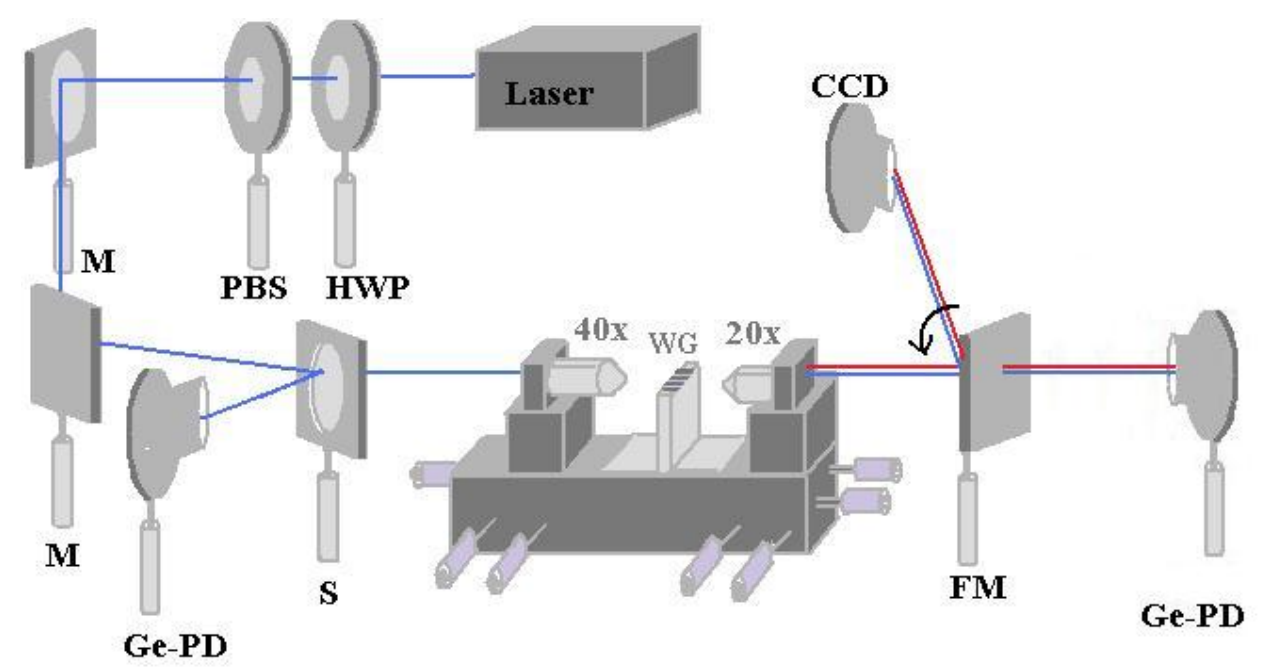

Figure 6.1 - Schematic of the experimental setup for loss measurement: HWP, half-wave plate; PBS, polarization beam splitter; S, sampler; M, mirror; WG, waveguide; FM, flip mirror; PD, photodetector; OSA, optical spectrum analyzer; CCD, charge coupled device (camera).

\subsection{Observation of Second Order Nonlinear Interac- tion}

In Chapter 4, phase matching simulations were carried out using the refractive index model extracted from Gehrsitz et al. [55]. For type I phase matching, the refractive indices of a TE fundamental mode at $1523.2 \mathrm{~nm}$ and a second harmonic TM mode at 


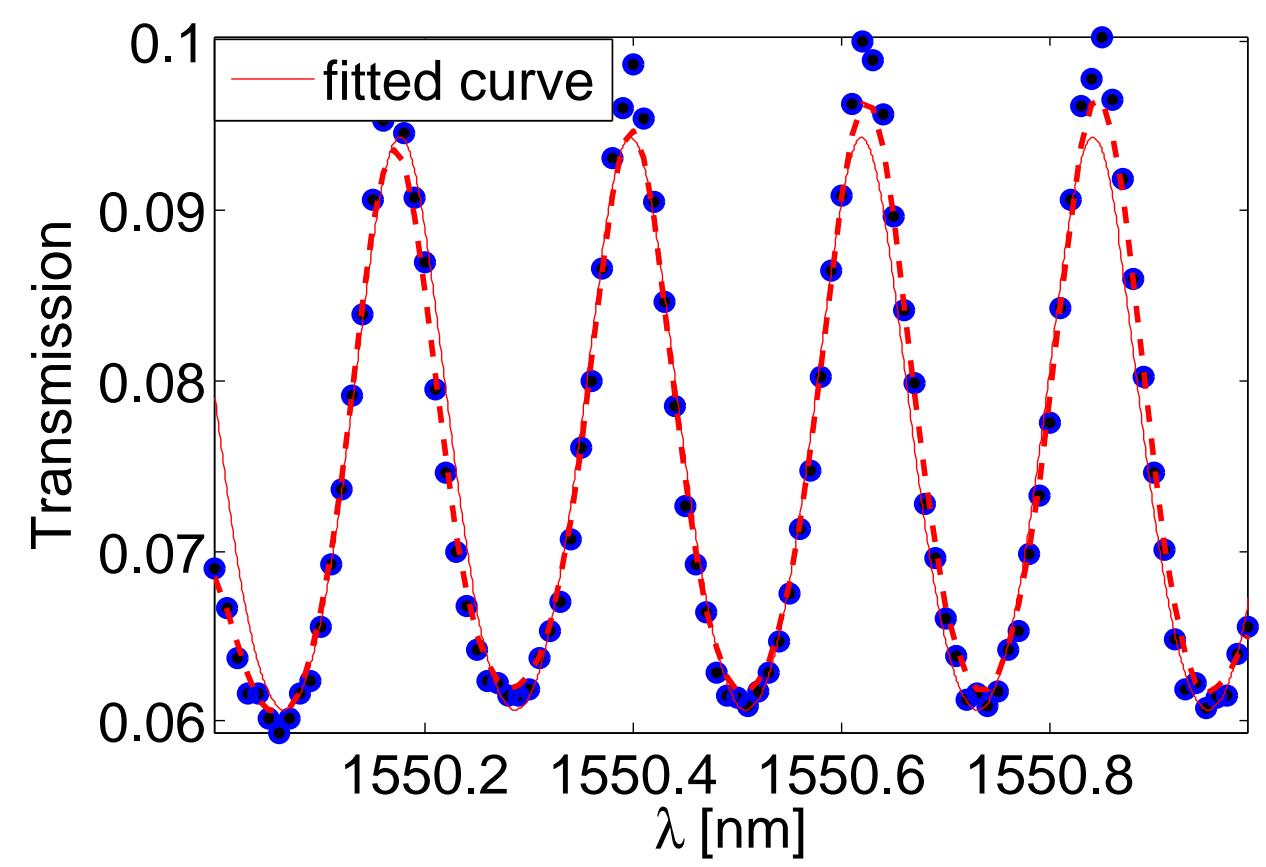

Figure 6.2 - plot of the Fabry-Perot transmission spectrum of a 1.62mm long waveguide; experimental measurements are shown in blue, theoretical fit is shown in red.

$761.6 \mathrm{~nm}$ were equal. Type II phase matching was achieved between TE/TM fundamental modes at $1540 \mathrm{~nm}$ and a TE second harmonic mode at $770 \mathrm{~nm}$. The characterization of the waveguides was therefore also carried out at the same wavelengths to examine the agreement between theory and experiment.

The best performing waveguides were characterized for second harmonic generation using three light sources; the first source was a 1.8 picosecond pulsed optical parametric oscillator, "MIRA-OPO by Coherent", pumped by a mode-locked Ti:Sapphire with a repetition rate of $76 \mathrm{MHz}$. The second source was a tunable 20 picosecond fiber laser, "WA programmable laser by Genia Photonics", with a repetition rate of $15 \mathrm{MHz}$. The third source was a tunable single-mode continuous-wave (CW) laser, "Velocity TLB-6300 tunable laser by New Focus". All sources were used with an end-fire coupling setup.

The devices were pumped using these three sources, and the internal input fundamental wave power as well as the output fundamental and generated second harmonic 


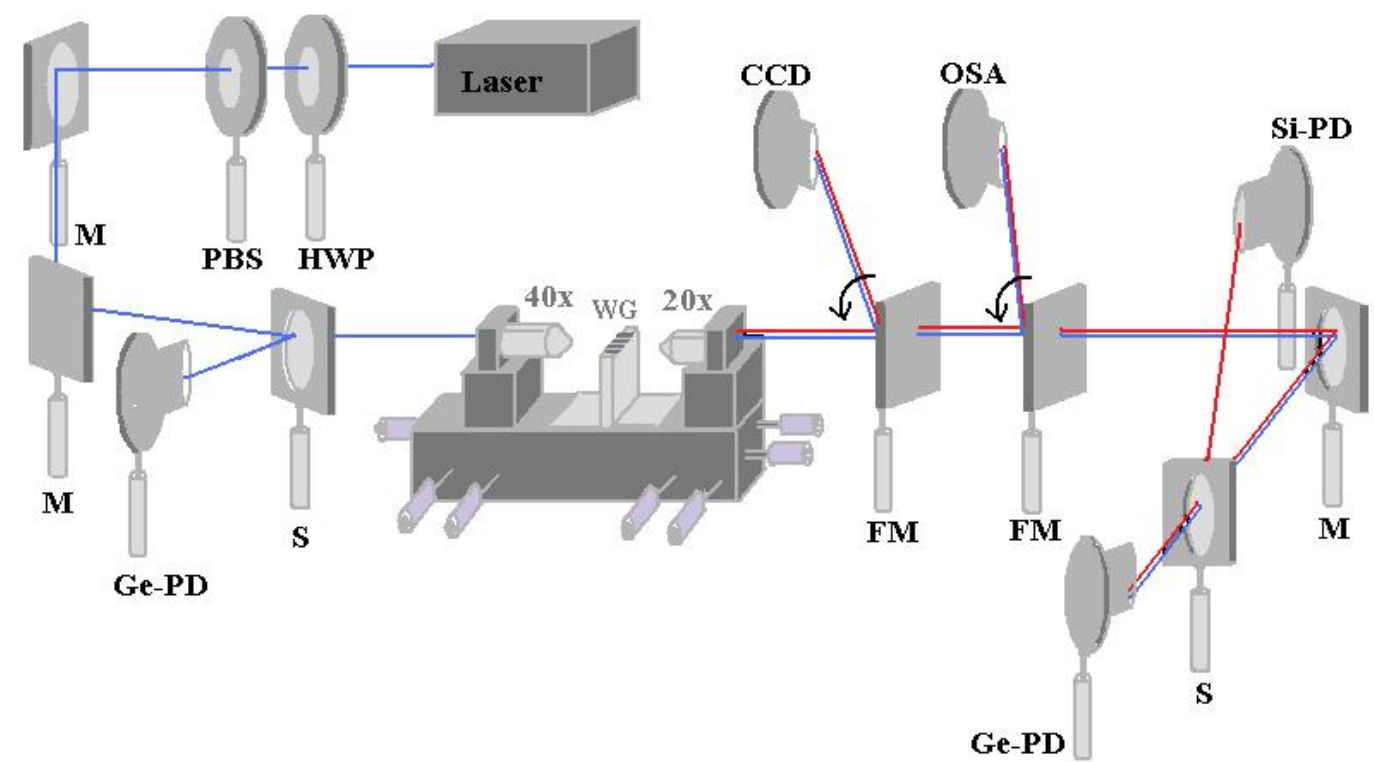

Figure 6.3 - Schematic of the SHG experimental setup: HWP, half-wave plate; PBS, polarization beam splitter; S, sampler; $M$, mirror; WG, waveguide; FM, flip mirror; PD, photodetector; OSA, optical spectrum analyzer; CCD, charge coupled device (camera).

waves were measured. Fig 6.3 shows a schematic of the set-up. The set-up is very similar to that of loss measurement with the exception of an optical spectrum analyzer (OSA), "561-FCH by Newport", for recording the spectra of the pulsed sources and a Silicon photo-detector for measuring the second harmonic output power. The laser beam first passes through a HWP and a PBS for polarization control. The input pump power is then measured using the Germanium photo-detector "Newport 883-IR". Next, the beam passes through an input objective lens and is incident on the facet of the waveguide. Second harmonic light is generated as this pump propagates through the waveguide. Both beams then exit the waveguide and are focused using an objective lens. Two flip mirrors on the path of the output beams reflect the light towards a CCD camera and the optical spectrum analyzer. If not reflected by the flip mirrors, the beams are then guided towards a sampler which further sends the beams to Germanium, "Newport 883-IR" and Silicon photo-detectors, "Newport 918D-SL-OD3",for measurement of the fundamental and second harmonic output powers, respectively. 
The internal input power measured is attenuated from the original external value. This attenuation is due to the input losses which originated from the input objective lens, a Fresnel reflection coefficient of $26 \%$ at the device facet and a mode input coupling factor of $18 \%$ between the incident beam and the TIR spatial profiles.

The wavelength at which phase matching occurs was determined by tuning the source wavelength and observing the second harmonic power. The tuning curve for all three sources is shown in Fig. 6.4. Clear peaks in the second harmonic power indicate phase matching at the corresponding wavelengths.

As discussed in Chapter 4, the two dimensional nature of the waveguides in experiments causes changes in the phase matching wavelengths from those originally designed in $1 \mathrm{D}$. According to the two dimensional simulations, the new wavelengths are shifted towards longer wavelengths, and this shift is increased as the width of the ridge waveguide is decreased. Fig. 6.4 shows the tuning curves for ridge widths of about $2.76 \mu \mathrm{m}$ as they were the least lossy among the available waveguides. The phase matching wavelengths for both type I and type II are indeed shifted to longer than what 1D theory predicted.

The second harmonic wave power was monitored as a function of the input pump power at the phase matching wavelength in order to confirm the quadratic relationship between these powers. Figure 6.6 shows plots of the $\mathrm{SH}$ power versus pump power on a logarithmic scale. The slope of these graphs indicates the order of the polynomial relationship between the powers. Indeed, the same procedure done at a point away from the phase matching wavelength would not show the same quadratic behaviour. An example of this is seen in Fig. 6.7.

From the tuning curves, the loss of the second harmonic wave can be extracted. The loss values vary according to the power level and are listed in table 6.1 for the three sources. These loss values were obtained by fitting the tuning curves of Fig. 6.4 to a Lorentzian function and associating the SH loss with the FWHM bandwidth of the Lorentzian [56]. 

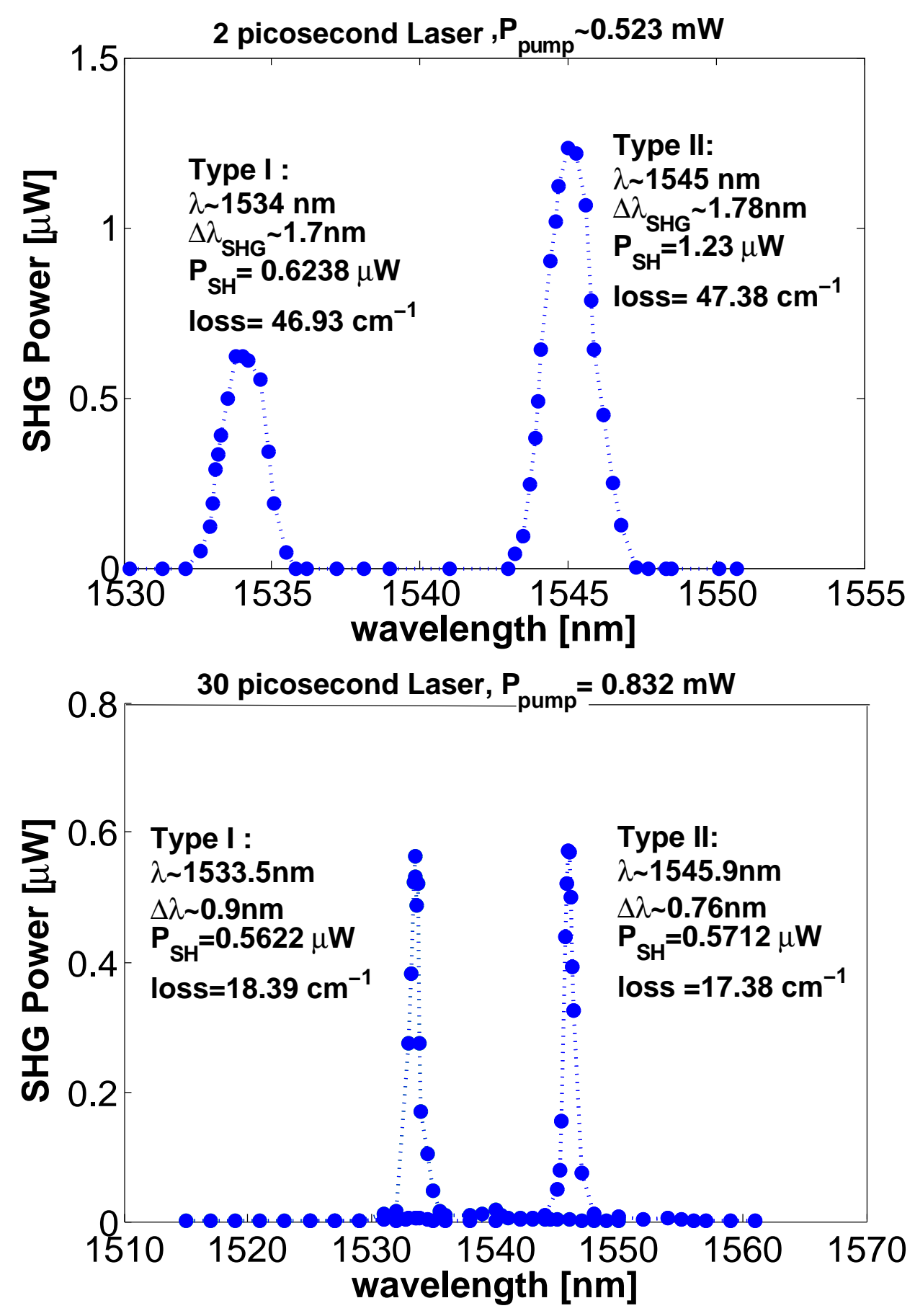


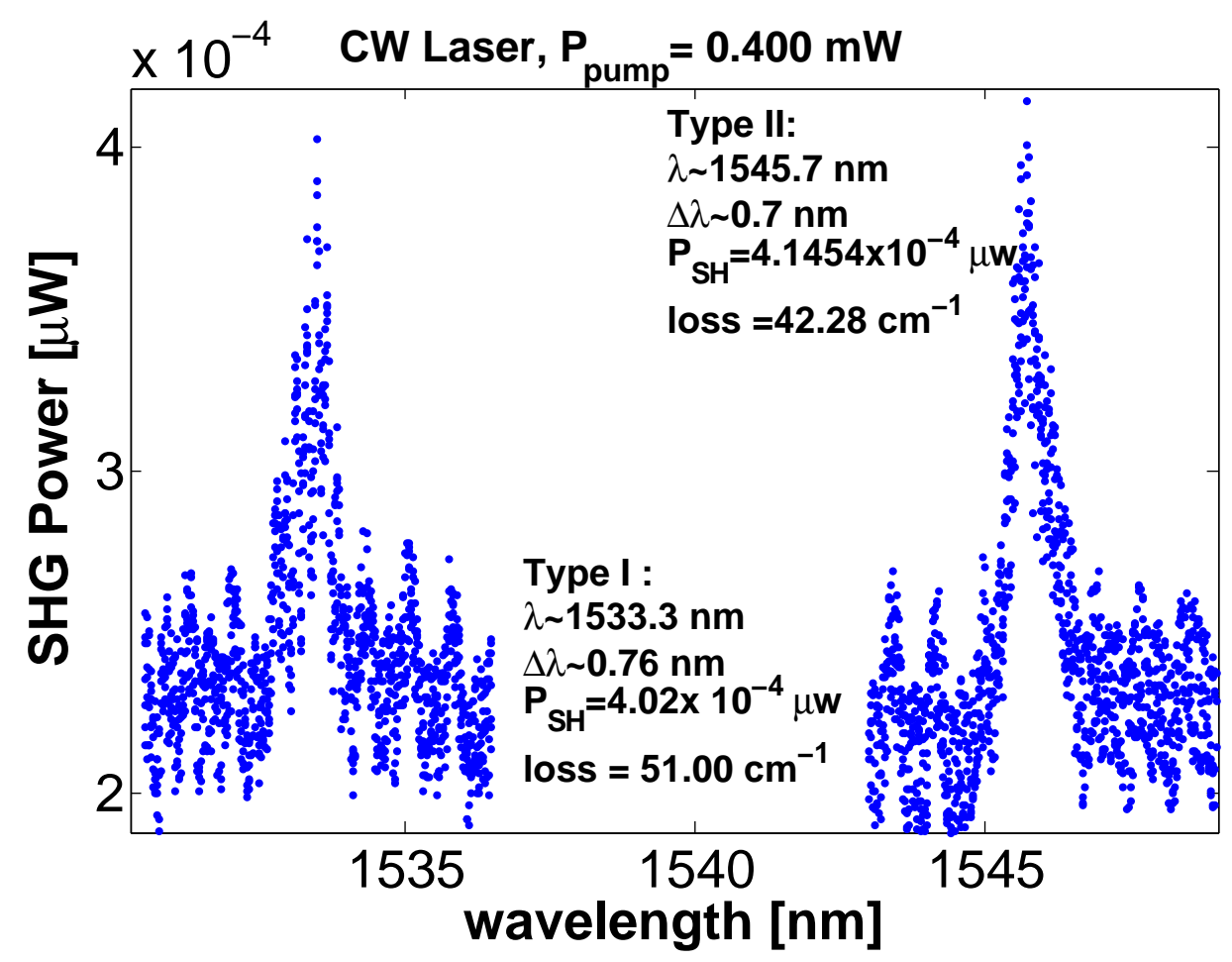

Figure 6.4 - SH power as a function of tuned pump wavelength for the (top) 1.8 ps, (center) 20 ps and (bottom) CW sources for both type I and type II phase matching.

Table 6.1 - Comparison of Wave Parameters for Different Multilayer Designs.

\begin{tabular}{|l|l|l|l|}
\hline Loss $\left(\mathrm{cm}^{-1}\right)$ & $1.8 \mathrm{ps}$ Source & 20 ps Source & CW Source \\
\hline Type I & 46.93 & 18.39 & 51.00 \\
\hline Type II & 47.38 & 17.38 & 42.28 \\
\hline
\end{tabular}

The device normalized conversion efficiency defined by Eq. 3.3 can also be estimated from the internal fundamental and second harmonic power levels; this normalized conversion efficiency was estimated to be $4.14 \times 10^{3} \% \mathrm{~W}^{-1} \mathrm{~cm}^{-2}, 2.0153 \times 10^{3} \% \mathrm{~W}^{-1} \mathrm{~cm}^{-2}$ and $6.7647 \% \mathrm{~W}^{-1} \mathrm{~cm}^{-2}$ for type II phase matching for the three sources respectively characterized for the waveguides with the lowest loss.

The difference in the efficiency values is associated with the difference in the corresponding peak powers of the three sources, with the peak power of the 1.8 ps source being 


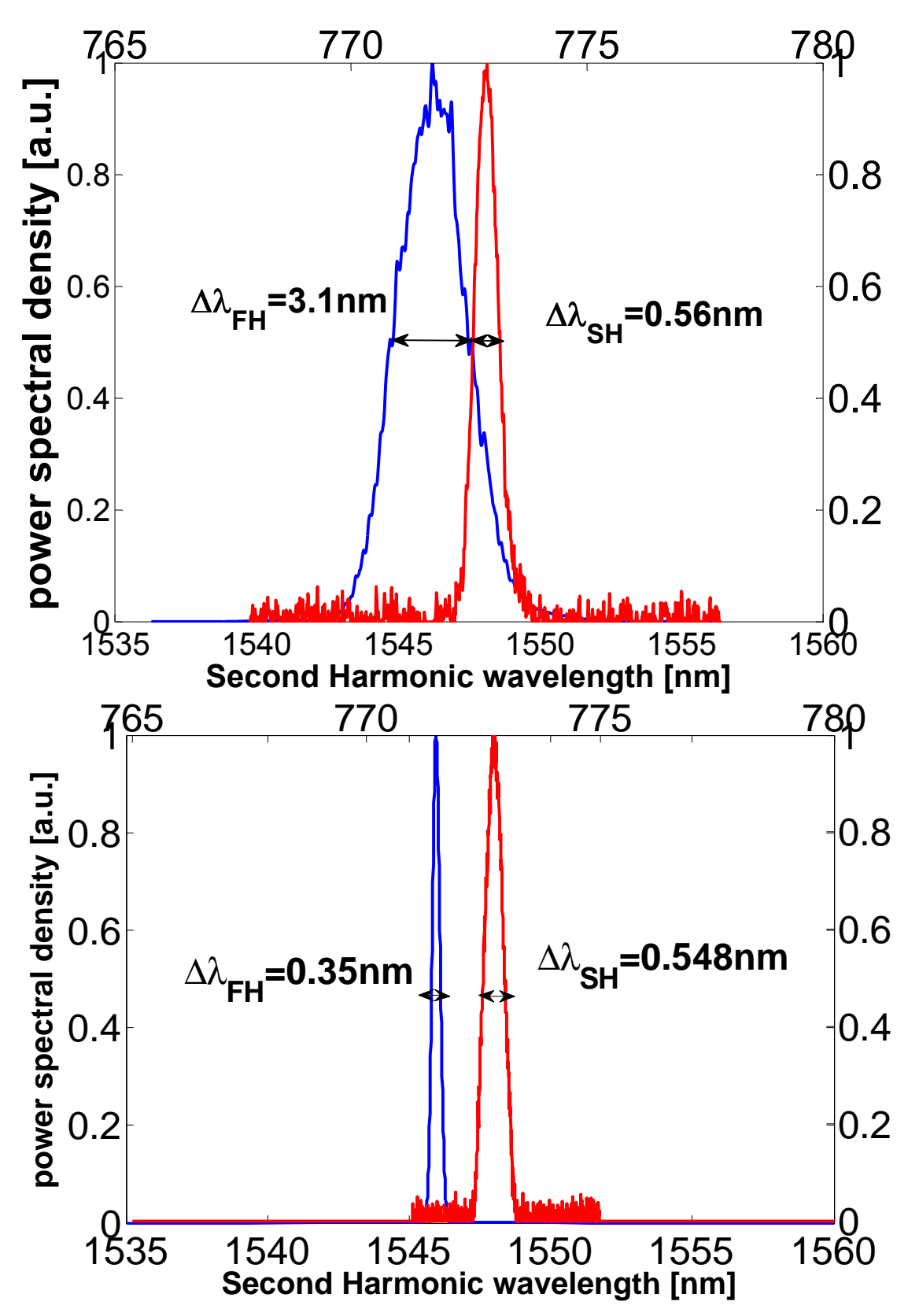

Figure 6.5 - Spectra of pump and the corresponding SH for type II phase matching for the $1.8 \mathrm{ps}$ (top) and $20 \mathrm{ps}$ (bottom) sources; the spectral intensities are in arbitrary units (a.u). 

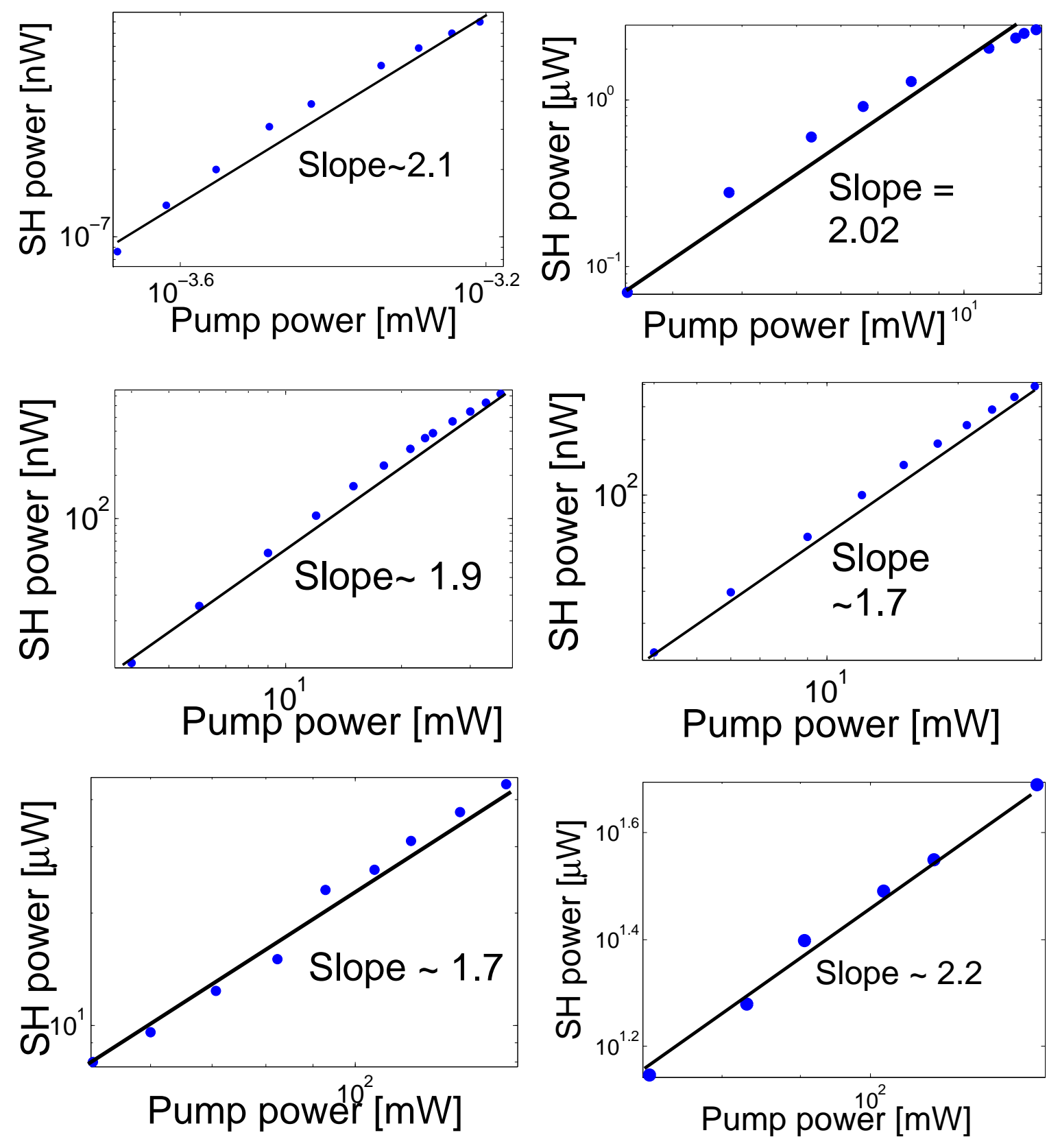

Figure 6.6 - Quadratic relationship between the SH and pump powers for the 1.8 ps(top), 20 ps (center), and CW (bottom) sources for type I (left) and type II (right) phase matching schemes. 


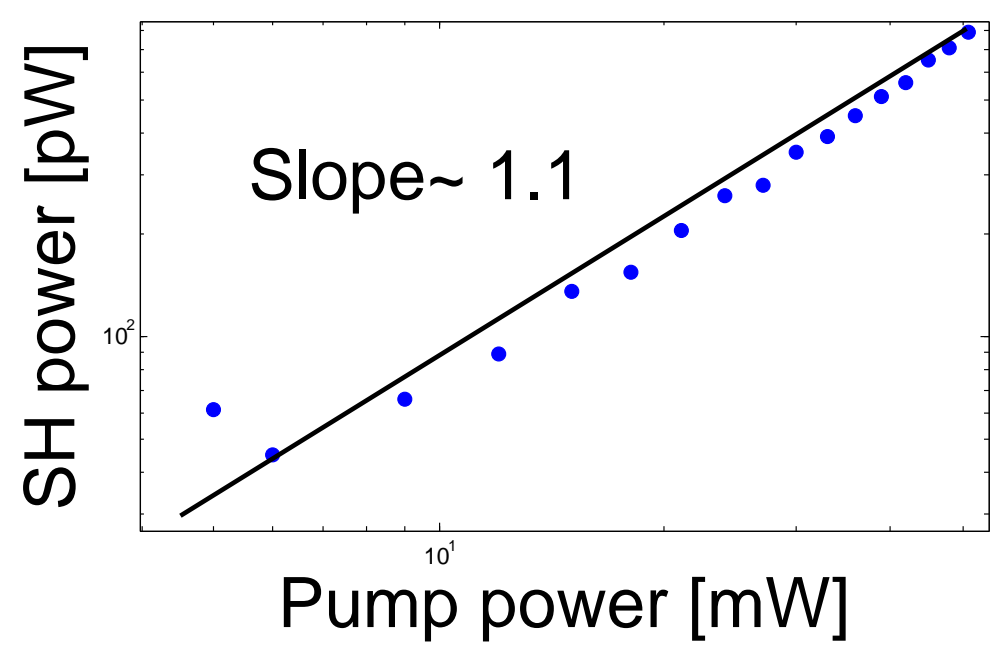

Figure 6.7 - Plot of the linear relationship between SH and pump power for type II phase matching for the 1.8 ps laser source at a wavelength of $1531 \mathrm{~nm}$, away from the phase matching point.

the greatest and that of the CW source, the lowest; however, the ratio of the peak powers is higher than that of the conversion efficiencies. This difference in the ratios originates from greater losses in the pulsed regime due to two-photon absroption of the fundamental beam in comparison to the continuous wave regime. Also, for the same source, the conversion efficiency values achieved using interface modes in this work are an order of magnitude smaller in comparison to those achieved so far by BRWs; nonetheless, the presence of this new type of wave provides additional degrees of freedom in the design of second order nonlinear processes. Furthermore, process bandwidth tunability in such a device is more accessible and tenable through the control over waveguide properties. This can be a key parameter in applications where the quantum optical properties of photons needs to be controlled [57].

Lastly, the spectra of both the fundamental and the second harmonic waves are shown in Fig. 6.5 for the 1.8 ps and 20 ps pulsed sources. The bandwidths of the FH and the SH waves were measured to be $3.1 \mathrm{~nm}$ and $0.56 \mathrm{~nm}$ respectively for the $1.8 \mathrm{ps}$ source and $0.35 \mathrm{~nm}$ and $0.548 \mathrm{~nm}$ for $20 \mathrm{ps}$ source. The sources can be assumed to be fourier 
transform limited, and hence the ratio between the pulse durations in time is expected to be inversely proportional to the ratio of the spectral widths of the pulses in the frequency domain. In practice, the pulse ratio is $1.8 / 20=0.09$ and the width ratio is $0.35 / 3.1=0.11$, which shows agreement with theory.

The observation of nonlinear interaction in agreement with theoretical predictions in the designed waveguide structures verifies the existence of coupled interface modes in periodic layered media. The demonstration of second harmonic generation using coupled interface modes further proves the capability of these modes to be utilized in phasematched nonlinear processes. Second order frequency mixing processes generating near and mid-infrared light can occur in much the same way as second harmonic generation in these structures. 


\section{Chapter 7}

\section{Conlcusions}

In this chapter, a brief review of the work done in the course of this thesis is provided and some future outlooks on the researched topic are discussed.

\subsection{Summary}

This thesis has reviewed interface modes, and developed a new type of electromagnetic interface mode to be utilized in nonlinear interaction with other modes in a periodic layered medium. The mode has been theoretically analyzed and its behaviour for second order nonlinear interaction has been studied. The waveguide structure in which it propagates has been fabricated and linear and nonlinear properties of the mode have been examined.

The proposed mode consists of two interface modes existing each on one side, at the boundary of a homogenous core and a capped periodic layered medium, coupled at the center of the core. Dispersion equations guiding the behaviour of the modes were developed and band diagrams of frequency versus wave vector for the mode were studied.

The modes were next studied for their application in a second order nonlinear optical process. Second harmonic generation involves a simpler experimental set-up in comparison to those of other three wave mixing processes. Hence, second harmonic generation 
was chosen as the process through which the utilization of these modes in nonlinear interaction could be experimentally demonstrated. The phase-matching condition ensuring efficient nonlinear interaction was achieved via a technique developed for matching the momentum vector of interacting waves in Bragg reflection waveguides. The method takes advantage of the dispersion properties of a periodic layered structure to match the phases of a mode guided through total internal reflection and in this case, the coupled interface mode.

Critical nonlinear process parameters such as conversion efficiency, group velocity dispersion and group velocity mismatch between the interacting waves, were studied and the effects of structure properties such as material refractive index and thickness on these parameters were examined; optimum values were considered in designing a structure for type II phase matching of SHG. The wavelengths at which phase matching would occur were theoretically predicted and two dimensional designs were simulated to more accurately model the structure.

A GaAs/AlGaAs wafer was grown and waveguides were fabricated according to the theoretical designs for type II phase matching. Using optimized AlGaAs patterning recipes including oxide deposition, photoresist deposition, patterning and development, oxide etching, wafer etching, and sample thinning, ridge waveguides were fabricated in a variety of ridge widths.

The waveguides were then characterized for linear and nonlinear properties. Loss measurements and SHG experiments were performed. Typical loss values of about 2.71 $\mathrm{cm}^{-1}$ were obtained for waveguide ridge widths of about $2.65 \mu \mathrm{m}$. Three light sources were used to demonstrate second harmonic generation and to compare the behaviour of the generated second harmonic waves. The agreement of theory and experiment on the second harmonic wavelengths generated for the different phase matching types was the main proof of the existance of coupled interface modes and their utilization in the nonlinear process. 
Lastly, a conversion efficiency value of $4.14 \times 10^{33} \% \mathrm{~W}^{-1} \mathrm{~cm}^{-2}$ was obtained for type II phase matching in the waveguides with the lowest losses. This project aimed at and successfully established second harmonic generation in periodic layered media, using coupled interface modes. This was the first demonstration of such phenomenon, and the next section will be discussing the implications and future considerations regarding this study.

\subsection{Future Work}

In the work of this thesis, coupled interface modes were studied with the aim of removing some of the limitations of conventional Bragg reflection waveguides. In particular, more command of properties such as group velocity dispersion and group velocity mismatch was sought. The proposed designs in this study can further be optimized by a more extensive scan of the parameter space for better control over the aformentioned parameters.

\section{-Three wave mixing processes beyond SHG}

The addition of a third type of mode supported within the same structures where TBR and TIR modes exist, provides the opportunity to explore three wave mixing processes other than second harmonic generation, such as sum frequency generation and difference frequency generation, with better coupling between the modes; the utilization of CIMs along with TBR modes in periodic layered media is another topic to examine in the future since it will benefit from better modal overlap than either of the two modes have had with TIR modes. The similar mode profiles of CIMs and TBR modes are the reason for this better overlap which translates to greater nonlinear conversion efficiency for second order nonlinear processes. This in fact was one of the main motivations behind the work of this project. 


\section{-Fabrication}

The characterization results achieved in this work have been greatly affected by the fabrication process. Due to a high aluminium concentration contrast between the $85 \% \mathrm{Al}$, AlGaAs buffer layer stacked on top of the $30 \% \mathrm{Al}$, AlGaAs cladding layer and the choice of growth temperatures, the fabricated wafer suffered from an increased surface defect density in comparison to previous designs of layered AlGaAs wafers. Consequently, it was only feasible to fabricate very few samples with low defect densities, chosen carefully from the center of the wafer. This in turn removed the opportunity to fruther optimize the fabrication recipe for the purposes of the particular design.

These defects can be avoided by eliminating the last cladding layer of the bottom stack and optimizing the MBE growth temperatures. With a more optimized fabrication recipe to ensure favorable $\mathrm{PR}$ mask deposition and required etch depths, the losses of the interacting waves could be minimized and greater nonlinear efficiencies could be observed.

\section{-Mode Far Field}

Phase matching using modes in semiconductor periodic layered media was originally introduced to achieve monolithic integration of active and passive devices on the same chip. Determining and controlling the far field of the analyzed modes is therefore of particular importance. For instance, single lobed far field modes provide great convenience in mode couling between the nonlinear section and other devices such as fibers and lasers for instance. The far field of BRWs have only recently been analyzed [58], and their behaviour in comparison with that of the far field of TIR modes have been examined. Similar work could be done on coupled interface modes. The far field of CIMs could be studied both theoretically and experimentally to better understand and utilize them for more efficient device integration. It would also be of interest to examine the similarities and differences of the far field performance between CIMs and TBR modes. 


\section{Bibliography}

[1] S. V. Rao, K. Moutzouris and M. Ebrahimzadeh, "Nonlinear frequency conversion in semiconductor optical waveguides using birefringent, modal and quasi-phasematching techniques," Journal of Optics a-Pure and Applied Optics, vol.6, pp. 569-584, 2004.

[2] J. Carr; M.Y.P. Desmulliez; N. Weston; D. McKendrick ; G. Cunningham; G. McFarland; W. Meredith; A. McKee; C. Langton, "Miniaturised optical encoder for ultra precision metrology systems," Precision Engineering, vol.33, pp. 263-267, 2009.

[3] Porzio, A., V. D’Auria, et al., "Quantum communication exploiting above threshold OPO intensity correlations and polarization encoding," Optics and Lasers in Engineering vol. 45, pp 463-467, 2007.

[4] M. Jager, G. I. Stegeman, M. C. Flipse, M. Diemeer and G. Mohlmann, "Modal dispersion phase matching over $7 \mathrm{~mm}$ length in overdamped polymeric channel waveguides," Applied Physics Letters, vol.69, pp. 4139-4141, 1996.

[5] A. Fiore, S. Janz, L. Delobel, P. van der Meer, P. Bravetti, V. Berger, E. Rosencher, "Second-harmonic generation at $\lambda=1.6 \mu m$ in AlGaAs/Al2O3 waveguides using birefringence phase matching," Appl. Phys. Lett., vol. 72, pp. 2942-2945, 1998.

[6] C. B. Ebert, L. A. Eyres, M. M. Fejer, and J. H. Harris, "Measurement of the nonlinear coefficient of orientation-patterned aAs and demonstration of highly efficient second-harmonic generation," J. Cryst. Growth, vol. 227, pp. 628-630,1999. 
[7] Robert W. Boyd , "Nonlinear Optics," Academic Press, pp. 109, 85, 2008.

[8] A. S. Helmy, D. C. Hutchings, T. C. Kleckner, J. H. Marsh, A. C. Bryce, J. M. Arnold, C. R. Stanley, and J. S. Aitchison,C. T. A. Brown, K. Moutzouris and M. Ebrahimzadeh, "Quasi phase matching in GaAs- AlAs superlattice waveguides via bandgap tuning using quantum well intermixing," Opt. Lett., vol. 25, pp. 1370-1372, 2000 .

[9] P. Abolghasem and A. S. Helmy, "Matching layers in bragg reflection waveguides for enhanced nonlinear interaction," IEEE J. Quantum Electron., vol.45, pp. 646-653, 2009.

[10] A. S. Helmy, "Phase matching using Bragg reflection waveguides for monolithic nonlinear optics applications," Opt. Express, vol. 14. pp. 1243-1252, 2006.

[11] B. R. West and A. S. Helmy, "Analysis and design equations for phase matching using Bragg reflection waveguides," IEEE J. Sel. Top. Quantum Electronics, vol. 12, pp. 431-442, 2006.

[12] A. S. Helmy, B. Bijlani and P. Abolghasem, "Phase matching in monolithic bragg reflection waveguides," Opt. Lett., vol.32, pp. 2399-2401, 2007.

[13] P. Abolghasem, J. B. Han, B. J. Bijlani, A. Arjmand and A. S. Helmy, "Highly efficient second-harmonic generation in monolithic matching layer enhanced alxga1xas bragg reflection waveguides," Ieee Photonics Technology Letters, vol.21, pp. 1462-1464, 2009.

[14] M. Piliarik, M. Vala, I. Tichy and J. Homola, "Compact and low-cost biosensor based on novel approach to spectroscopy of surface plasmons," Biosensors \& Bioelectronics, vol.24, pp. 3430-3435, 2009. 
[15] R. Y. He, C. Y. Lin, Y. D. Su, K. C. Chiu, N. S. Chang, H. L. Wu and S. J. Chen, "Imaging live cell membranes via surface plasmon-enhanced fluorescence and phase microscopy," Opt. Express, vol.18, pp. 3649-3659, 2010.

[16] M. I. D'yakonov, "New type of electromagnetic wave propagating at an interface," Sov. Phys. JETP, vol. 67, 714-716, 1988.

[17] O. Takayama, L. Crasovan, D. Artigas and L. Torner, "Observation of dyakonov surface waves," Physical Review Letters, vol. 102, 2009.

[18] O. Takayama, L. C. Crasovan, S. K. Johansen, D. Mihalache, D. Artigas and L. Torner, "Dyakonov surface waves: A review," Electromagnetics, vol. 28, pp. 126$145,2008$.

[19] J. A. Polo and A. Lakhtakia, "Review of surface-wave propagation at the planar interface of a columnar or chiral sculptured thin film and an isotropic substrate ," Nanostructured Thin Films, vol. 7041, pp. E410-E410, 2008.

[20] Yeh, Pochi and Yariv, Amnon and Cho, A. Y., "Optical surface waves in periodic layered media," Applied Physics Letters, vol. 32, pp. 104-105, 1978.

[21] A. Yariv and P. Yeh, "Optical waves in crystal propagation and control of laser radiation," Wiley, 1984.

[22] M. Liscidini and J. E. Sipe, "Enhancement of diffraction for biosensing applications via bloch surface waves," Applied Physics Letters, vol.91, 2007.

[23] Pochi Yeh, "Optical Waves in Layered Media," New Jersey : John Wiley, 2005.

[24] M. Liscidini, M. Galli, M. Patrini, G. Dacarro, L. C. Andreani, D. Bajoni, C. Ricciardi, F. Giorgis, R. W. Loo, M. C. Goh, M. Shi, J. E. Sipe and Ieee, "Enhancing light-matter interaction via bloch surface waves for biosensing applications," IEEE, Transparent Optical Networkds, 2009. 
[25] A. Shinn and W. M. Robertson, "Surface plasmon-like sensor based on surface electromagnetic waves in a photonic band-gap material," Sens. Actuator B-Chem., vol.105, pp. 360-364, 2005.

[26] A. Shinn and W. M. Robertson, "Surface plasmon-like sensor based on surface electromagnetic waves in a photonic band-gap material," Sens. Actuator B-Chem., vol.105, pp. 360-364, 2005.

[27] F. Villa and J. A. Gaspar-Armenta, "Photonic crystal to photonic crystal surface modes: Narrow-bandpass filters," Opt. Express, vol.12, pp. 2338-2355,2004.

[28] Z. N. Wang and D. H. Liu, "Narrow band longitude mode selector of laser based on conjugated photonic crystals," Chinese Physics Letters, vol.26, 2009.

[29] B. R. West and A. S. Helmy, "Analysis and design equations for phase matching using Bragg reflection waveguides," IEEE J. Sel. Top. Quantum Electronics, vol. 12, pp. 431-442, 2006.

[30] B. J. Bijlani and A. S. Helmy, "Bragg reflection waveguide diode lasers," Opt. Lett., vol.34, , pp. 3734-3736, 2009.

[31] A. K. Ghatak, K. Thyagarajan, and M. R. Shenoy, "Numerical analysis of planar opticalwaveguides using matrix method," J. Lightw. Technol.,vol. T-5, pp. 660667,1987 .

[32] C. Vandenbem, "Electromagnetic surface waves of multilayer stacks: Coupling between guided modes and bloch modes," Opt. Lett., vol.33, pp. 2260-2262, 2008.

[33] W. M. Robertson, "Experimental measurement of the effect of termination on surface electromagnetic waves in one-dimensional photonic bandgap arrays," Journal of Lightwave Technology, vol.17, pp. 2013-2017, 1999. 
[34] E. Guillermain, V. Lysenko and T. Benyattou, "Surface wave photonic device based on porous silicon multilayers," J. Lumines., vol.121, pp. 319-321, 2006.

[35] F. Villa, L. E. Regalado, F. Ramos-Mendieta, J. Gaspar-Armenta and T. Lopez-Rios, "Photonic crystal sensor based on surface waves for thin-film characterization," Opt. Lett., vol.27, pp. 646-648, 2002.

[36] M. Ohashi, T. Kondo, R. Ito, S. Fukatsu, Y. Shiraki, K. Kumata and S. S. Kano, "Determination of quadratic nonlinear-optical coefficient of alxga1-xas system by the method of reflected 2nd harmonics," Journal of Applied Physics, vol.74, pp. 596-601, 1993.

[37] A. S. Helmy,D. C. Hutchings, T. C. Kleckner, J.H.Marsh, A. C. Bryce,J. M. Arnold, C. R. Stanley, J. S. Aitchison, C. T. A. Brown, K. Moutzouris,and M. Ebrahimzadeh, "Quasi phase matching in GaAsAlAssuperlattice waveguides via bandgap tuning using quantum well intermixing," Opt. Lett., vol. 25, pp. 13701372, 2000.

[38] Y. Sakurai and F. Koyama, "Control of group delay and chromatic dispersion in tunable hollow waveguide with highly reflective mirrors," Japanese Journal of Applied Physics Part 1-Regular Papers Short Notes 6 Review Papers, vol.43, pp. 5828-5831, 2004.

[39] S. Wagner, A. Al Mehairy, J. S. Aitchison, and A. S. Helmy, "Modelling and optimization of qausi-phase matching using domain disordering," IEEE J. Quantum Electron., vol. 44, pp. 424429, 2008.

[40] B. R.West and A. S. Helmy, "Dispersion tailoring of the quarter-wave Bragg reflection waveguide," Opt. Exp., vol. 14, pp. 40734086, 2006.

[41] Mode Solutions, ," Lumerical Solutions Inc., [computer aided design software], 20032010. 
[42] Arghavan Arjmand, A. Helmy "Towards the Fabrication of GaAs/AlGaAs Laser Diodes Using a Self-Aligned Process," Bachelor of Applied Science and Engineering thesis, 2008.

[43] S1818 Photoresist MSDS, http://kni.caltech.edu/facilities/msds/Shipley1818photoresist.pdf.

[44] MF-321 Developer MSDS, http://www.cnf.cornell.edu/msds/MF$321 \% 20$ Developer\%20\%2849323\%29.pdf.

[45] A. Narasimhan, "Thermal analysis of a silicon wafer processing combination bakechill station used in microlithography ," Materials and Manufacturing Processes, pp 273- 286, Vol. 20, No. 2 , 2005.

[46] Shul, R.J.a; Vawter, G.A.a; Willison, C.G.a; Bridges, M.M.a; Lee, J.W.b; Pearton, S.J.b; Abernathy, C.R., "Comparison of plasma etch techniques for IIIV nitrides," Solid-State Electronics, vol. 42, pp 2259-2267, 1998.

[47] Hosomi, K., Shirai, M.; Hiruma, K., Shigeta, J., Katsuyama, T., "AFM characterization of GaAs-AlGaAs waveguides," IEICE Transactions on Electronics, vol.E79-C, pp.1579- 1585, 1996.

[48] Amr.S.Helmy, Bhavin Bijlani, Study on SiO2 etch using a DOE, Department of electrical engineering, university of Toronto, 2006.

[49] Donald A.Neaman, "Semiconductor physics and devices," university of Mexico, pp.81-82, 2003.

[50] Franz, Gerhard; Hoyler, Charlotte; Kaindl, Josef, "Reactive ion etching GaAs and AlAs: Kinetics and process monitoring," Journal of Vacuum Science \& Technology B: Microelectronics and Nanometer Structures, vol. 14, pp.126-131, 1996.

[51] Sami franssila, "Introduction to Micro fabrication," Johnley and Sons, Paperback, pp. 126-127, 2004. 
[52] M.Sugawara, "Plasma Etching: Fundamentals and Application," Oxford University Press, pp.67-68, 1998.

[53] Su Jianxiu, Chen Xiqu, Du Jiaxi and Kang Renke, "Material removal rate in chemical-mechanical polishing of wafers based on particle trajectories," Journal of Semiconductors, vol.31, pp. 0560021-0560026, 2010.

[54] S. Taebi, M. Khorasaninejad and S. S. Saini, "Modified fabry-perot interferometric method for waveguide loss measurement," Appl. Optics, vol.47, pp. 6625-6630, 2008.

[55] S. Gehrsitz, F. K. Reinhart, C. Gourgon, N. Herres, A. Vonlanthen and H. Sigg, "The refractive index of alxga1-xas below the band gap: Accurate determination and empirical modeling," Journal of Applied Physics, vol.87, pp. 7825-7837, 2000.

[56] 1A. Fiore, S. Janz, L. Delobel, P. van der Meer, P. Bravetti, V. Berger, E. Rosencher, and J. Nagle, "Second-harmonic generation at $\lambda=1.6$ in AlGaAs/Al O waveguides using birefringence phase matching," Appl. Phys. Lett., vol. 72, pp. 29422944, 1998.

[57] P. Abolghasem, M. Hendrych, X. J. Shi, J. P. Torres and A. S. Helmy, "Bandwidth control of paired photons generated in monolithic bragg reflection waveguides," Opt. Lett., vol.34, pp. 2000-2002, 2009.

[58] Nima Zareian, Amr S. Helmy, " Far Field of Bragg Reflection Waveguides:Characteristics and Closed-Form Approximation," J. Lightwave Technology - Submitted. 